\title{
Playing with Pleasure and Anxiety: \\ Dark Play, Transgression, and Kink in Grand Theft Auto 5
}

\section{by}

Emily Jean Ketterling

A thesis submitted to the Faculty of Graduate and Postdoctoral Affairs in partial fulfillment of the requirements for the degree of

Master of Arts

in

Law and Legal Studies

Carleton University Ottawa, Ontario

(c) 2018, Emily Jean Ketterling 


\section{Abstract}

This project aims to explore how the lessons learned about feminist media analysis in the sex wars can help us develop more nuanced understandings of transgressive video games. Radical feminist approaches to pornography tend to focus on harm and use media-effects research to support their claims; I argue that these same arguments are being played out in a new arena: anti-violence in video games advocacy. Using autoethnography, hermeneutical textual analysis and in-depth interviews, I aim to reread one particularly contentious title, Grand Theft Auto 5, in a way that acknowledges the complicated and contingent pleasures of transgression. Using a kink-informed lens, I push back against essentializing cause-and-effect readings of transgressive gameplay including GTA 5's interactive violence, rape fantasy, and cross-racial play. In doing so, I aim to present "not only a critical but a political interrogation" of radical feminist approaches to video games. ${ }^{1}$

\footnotetext{
${ }^{1}$ Clover, Men, Women, and Chain Saws.
} 


\section{Acknowledgments}

I owe endless thanks to my supervisor, Professor Ummni Khan, whose enthusiasm, support, and mentorship have been an invaluable buoy and source of motivation. Thank you to the other members of my committee: Professor Aubrey Anable and Professor Benjamin Woo, for their time, effort, and invaluable feedback. Thanks also to Professor Stacy Douglas and Professor Dale Spencer for their support and encouragement in the early stages of this project and my degree.

Thank you to my friends, family and colleagues who helped me throughout this project. In particular, I am grateful for Alexandra Killham's friendship, enthusiasm, and help in editing; and to my parents and sisters for their steadfast support and encouragement. Thank you to Chris for the support he provided throughout my degree, as well as for showing me how to build my gaming computer and set up the Let's Play technology. 


\section{$\underline{\text { Table of Contents }}$}

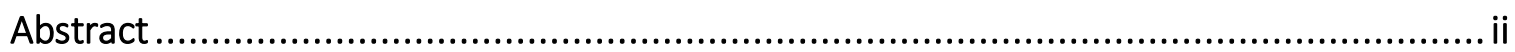

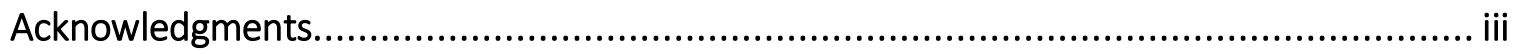

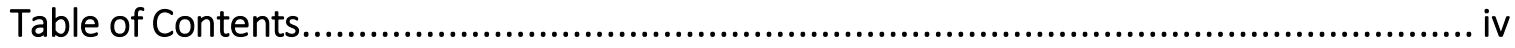

List of Figures ...................................................................................... vi

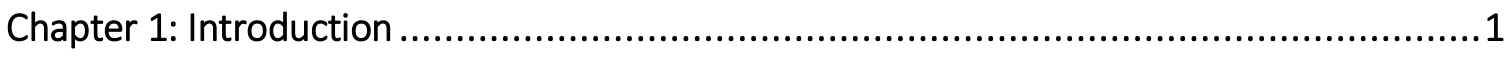

Chapter 2: Literature Review and Theoretical Background .................................. 7

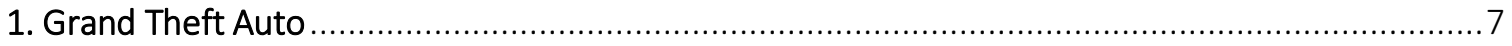

2. Feminism, violence and harm in pornography and video games............................................ 9

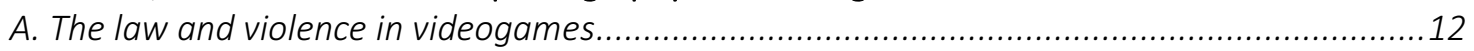

B. Media-effects models of porn and violent videogames ................................................... 17

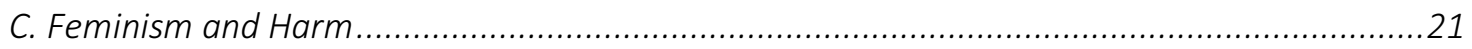

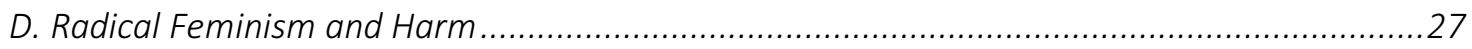

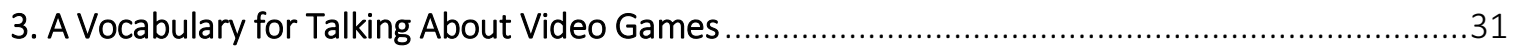

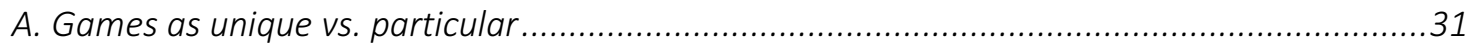

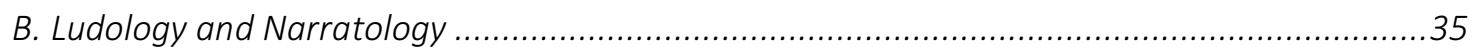

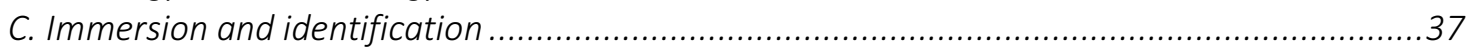

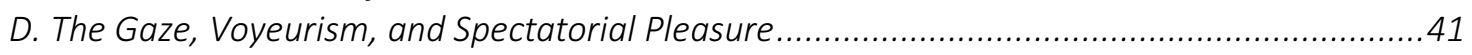

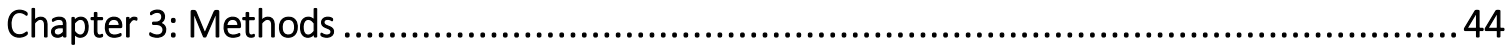

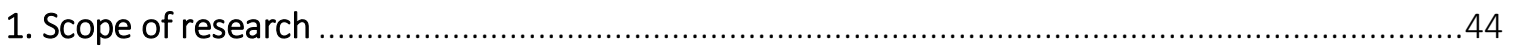

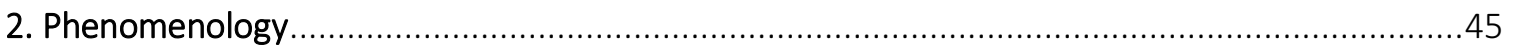

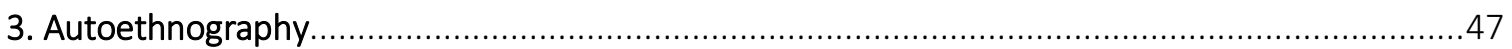

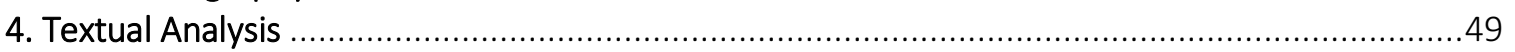

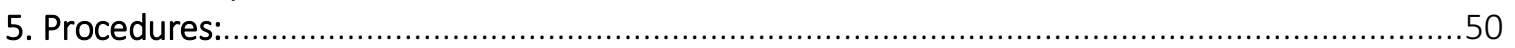

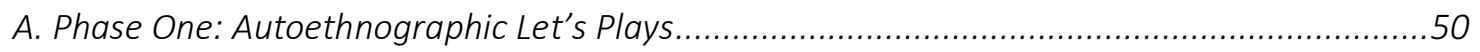

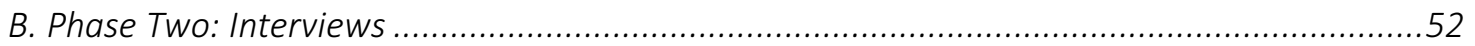

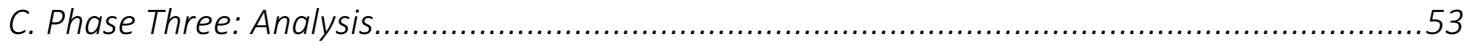

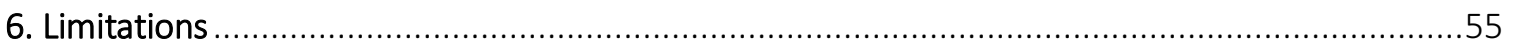

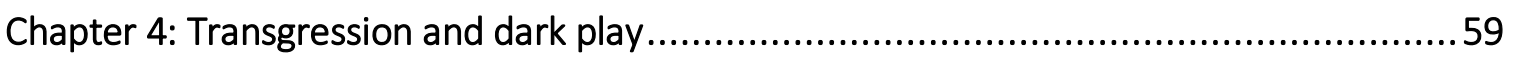

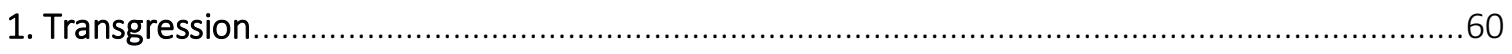

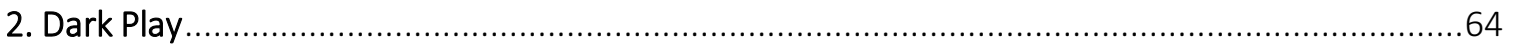

Chapter 5: BDSM, Violence, and Pleasure in the Uncanny Valley ............................ 75

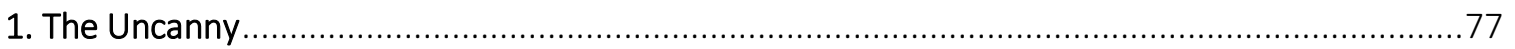

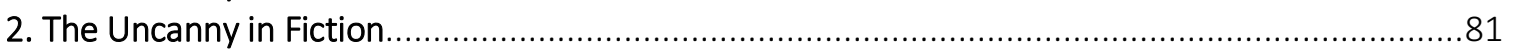

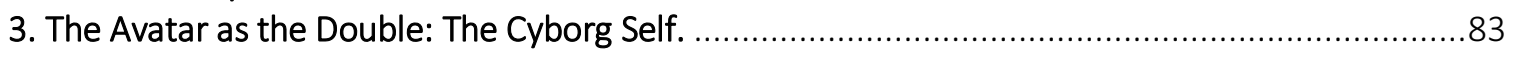

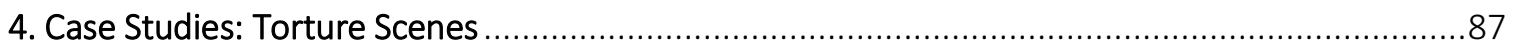

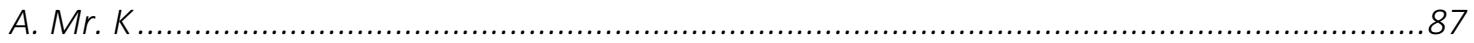

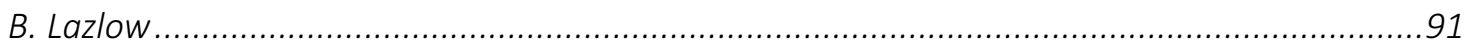

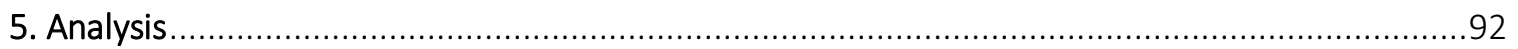

A. Violator/Violated: Dark Play and Identification in Liminal Space .........................................92

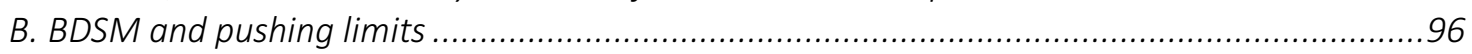




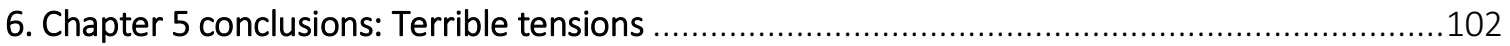

Chapter 6: Hood Safari: Digital Blackface and Race Play ....................................... 104

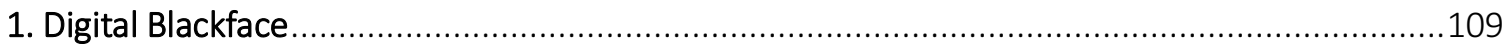

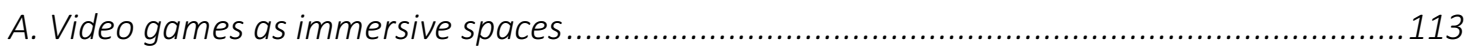

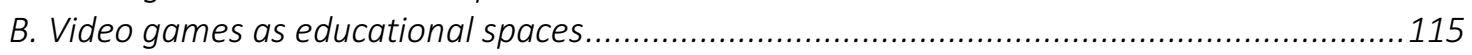

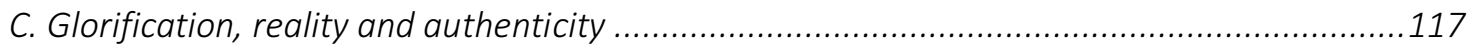

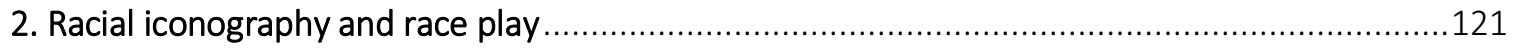

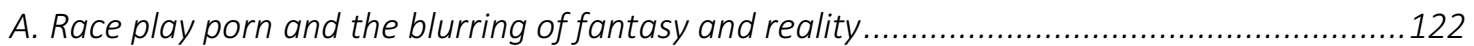

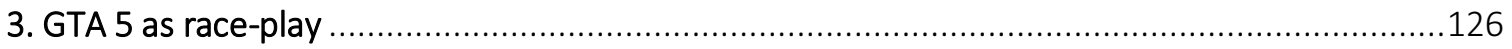

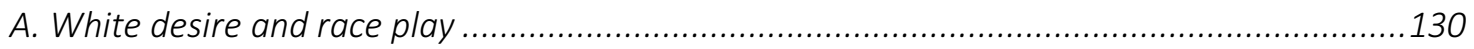

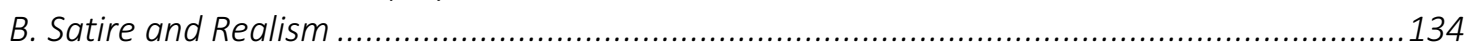

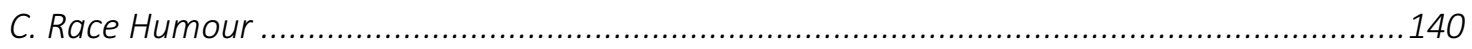

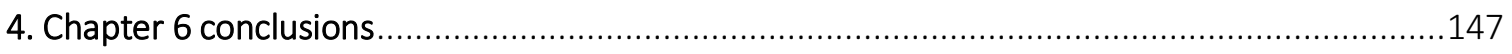

Conclusion: BUSTED! Re-reading GTA 5 for pleasure and anxiety............................ 149

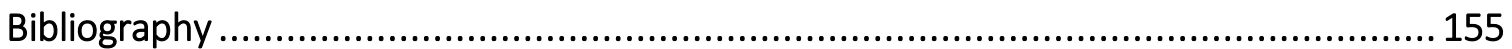

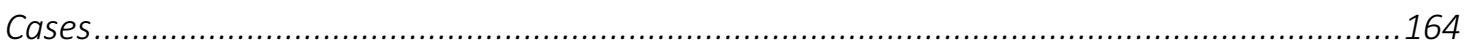

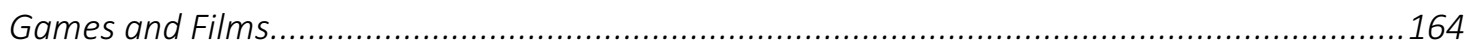




\section{List of Figures}

Figure 1: A screenshot from the original GTA, released in 1997. The photo depicts the "Busted!" message still used to indicate failure (death or capture by police). The apprehended player avatar stands next to several run-down pedestrians who are depicted as bloody splats on the sidewalk.

Figure 2: Let's Play videos show gameplay, a nested capture of the player, and allow for running commentary.

Figure 3: Figure illustrating the uncanny valley from translation of Masahiro's article. Original Caption reads: "The graph depicts the uncanny valley, the proposed relation between the human likeness of an entity, and the perceiver's affinity for it [Translator's note: Bunraku is a traditional Japanese form of musical puppet theater dating to the $17^{\text {th }}$ century. The Puppets range in size but are typically a meter in height, dressed in elaborate costumes, and controlled by three puppeteers obscured only by their black robes.] 


\section{Chapter 1: Introduction}

This project arose out of frustration with popular feminist analysis of violence in video games, which often mirrored essentializing radical feminist critiques of pornography. Watching Anita Sarkeesian's popular media criticism series Tropes vs. Women in Video Games was a key moment in my realization that while I had developed a relatively nuanced literacy about pornography, the same was not true of my understanding of violence in video games. This appeared to hold true more broadly with the public discourse about video games, which often seemed to replicate the tone, and concerns of the Sex Wars. As Albury suggests, both conservative and feminist opposition to pornography is often framed in moral terms, leading to analytic dead ends where the questions being asked are "either/or questions [that] demand yes/no answers". ${ }^{2}$ This leads to 'paranoid' close readings of texts where the reader is able to "triumphantly expose" messages and meanings in a text without considering how these interpretations might be different for different audience members. ${ }^{3}$ These readings claim to expose and reveal the truth about a text, while simultaneously denying their own subjectivity; drawing on Sedgewick, Albury calls this an "over-investment in the process of criticism". ${ }^{4}$

For example, Sarkeesian depends heavily on "showing" her viewers the true or authorial meaning of a text. She tends to brush aside the experience of the player, focusing instead on interpreting the game as a text that can be understood independent

\footnotetext{
2 Albury, "Reading Porn Reparatively," 648.

3 Sedgewick, Touching Feeling: Affect, Pedagogy, Performativity; Albury, "Reading Porn Reparatively," 648.

${ }^{4}$ Albury, "Reading Porn Reparatively," 648; Sedgewick, Touching Feeling: Affect, Pedagogy, Performativity.
} 
of the play experience. By ignoring contextualized experience, she attempts to erase the polysemic spectrum of meaning making, arguing that certain images or codes inherently signify violence and harm. This relies on a "one-to-one correspondence" between the signifier (for example, a female non-playable character (NPC) in a brothel) and its meaning (in this case, a woman's body being 'bought' and 'used'). ${ }^{5}$

While this project's focus has shifted slightly from violence to transgression in games, the central concern remains the same: how can we apply lessons learned during the "sex wars" about the analysis of pornography to the feminist analysis of new digital media and content that some may find distasteful, harmful, or abject? Therefore, in many ways I am riffing directly off of Nash's central question in her work on subverting the conventional analysis of black pornography and performers: is it possible to conduct a feminist project on violent and transgressive video games and to develop theoretical and political stances that eschew knee-jerk condemnation of images and narratives that, if taken literally, usually signify oppression or depravity? ${ }^{6}$ Further, are there readings of that allow us to recuperate these texts by using a kink-informed lens?

Reading for kinky pleasures can be a productive way of engaging with texts that feature power and violence, and is especially pertinent in reading video games which, as I will argue, have an inherently kinky structure. ${ }^{7}$ I am particularly interested in how this research question can help to untangle assumptions made about the impact of media on

\footnotetext{
${ }^{5}$ Lacombe, Blue Politics Pornography and the Law in the Age of Feminism, 39; Sarkeesian, Women as Background Decoration (Part 1).

${ }^{6}$ Nash, The Black Body in Ecstasy, 1.

7 Khan, "Fetishizing Music as Rape Culture."
} 
the user, and the way this is mobilized and understood in the legal understanding of transgression and media.

Based on autoethnographic and phenomenological analysis of Grand Theft Auto 5 (GTA 5), I argue that there is no one interpretation of transgressive moments in games. Contrary to analyses that rely on an essentializing reading of meaning, or a cause-effect relationship between violent media and real-life violence, I believe that meaning in video games is informed by a host of personal, political, social and cultural factors. As such, I show that games that are characterized as gratuitously violent are often significant to gamers in multitudes of ways. We can better understand these meanings by talking with them about their relationship to the game and to gameplay, and by understanding the cultural connection between transgression and taboo, and pleasure and anxiety.

Autoethnography and phenomenological research methods allow me to accomplish this because they foreground the embodied, situated player in an effort to understand how the game is perceived. ${ }^{8}$ I don't mean to ignore the use of prevalent violent, or sexist and racist tropes in games. Rather, I want to focus on player engagement with transgression as a multi-sensory experience that often extends beyond gameplay itself.

The project began with three framing questions: Are experiences of playing transgression/violence meaningful to game players? How does playing transgression/violence make players feel? What narratives of meaning do they construct regarding these experiences and how do these relate to their own personal experiences and lives? I believe that the following chapters help to elucidate the importance of

\footnotetext{
8 Keogh, A Play of Bodies, 13.
} 
subjective experience and play in answering each of these and provide the groundwork for further research on players' affective experience of transgression.

In Chapter 2 I briefly introduce GTA 5, before presenting a literature review focused on central debates in games studies and how they inform my research. The central argument of this chapter is that games are not unique, but rather have a set of particularities that makes them compelling vectors for experiencing transgression. I begin by outlining key debates and points of contention within game studies in order to build a vocabulary for my discussion of GTA 5. I then move on to discussing how radical feminism, feminist media studies, and media effects research has made sense of violence and transgression and how these debates have been taken up in the legal sphere. Finally, I discuss the importance of visual pleasure, the gaze and voyeurism before moving on to a brief introduction of the game itself.

In Chapter 3, I introduce my methodology and its theoretical underpinnings. This project takes a play-centric lens in attempting to understand how players experience GTA 5. While I use in-depth phenomenological interviews with male players, the backbone of the project is an autoethnography. Given the intimate nature of transgressive play, and the challenges of engaging in the sort of deep reflexivity needed for a successful phenomenological approach, autoethnography allowed me to develop my analysis in a way that was impossible through interviews alone. Of course, this makes this project highly subjective and reflective of my personal experience. I think that this subjective approach has value in relation to the goal of the research. By emphasising my subject position, I am able to complicate radical feminist analyses which imagines that, given that 
GTA 5 and media like it as presumed to be reserved for a male audience, women will either dislike or reject it, or that those women who do find it appealing are suffering from a false consciousness. Given this, I don't aim to produce generalizable results, other than to suggest that player experience of transgression is deeply personal, contingent, and subjective.

The subsequent chapters, which make up the substantive analytic component of my thesis are all centered loosely around the concept of transgression and dark play. As such, I introduce the theoretical framework I will use in Chapter 4. I begin by describing how interviewees and I made sense of playing GTA 5 as a transgressive experience. I then discuss transgression and dark play as theoretical concepts that tie together the next two chapters.

Chapter 5 concerns psychosexual pleasure, male masochism and the uncanny. This chapter is particularly concerned with exploring the dissolution of self that characterizes transgression and eroticism. Using phenomenological and autoethnographical analysis of my own playthrough of GTA 5's infamous torture scene(s), I aim to show how they can be experienced as both bodily violation and an erotic experience, and that this produces a state of liminality between violator and violated. I connect this to Freud's concept of the uncanny: the unconscious desires that must remain unacknowledged to support psychic organization, but that the structure of fictions, including video games, allow us to access.

Chapter 6 concerns race play and cross-racial performance. I examine the argument that playing black avatars is a sort of modern digital blackface, which upholds hegemonic 
racist ideology. Using my autoethnographic experience of race in GTA 5 as a departure point, I use Jennifer Nash's concept of racial iconography to re-read GTA 5's depiction of blackness through a kink-informed lens as a form of race-play. This sort of play is often characterized by both pleasure (even if these pleasures are problematic) and anxiety, and is dependent on an acknowledgement of racial dynamics, rather than their erasure. While critical race scholars are concerned about the ways that the presumed white player can play with race and temporarily perform otherness, I argue that there is analytic value in centering the pleasurable and erotic nature of race-play and race pleasure in games, even if (especially if!) these pleasures might be considered uncomfortable or unseemly. ${ }^{9}$ In this chapter I also discuss the role of race humour and satire in the game, arguing that rather than seeing the game as a direct simulation of reality, player balances in-game ethics and "real world" ethics in a form of dark play that augments the erotic and transgressive appeal of the humour.

\footnotetext{
${ }^{9}$ Leonard, "'Live in Your World, Play in Ours': Race, Video Games, and Consuming the Other," 8.
} 


\section{Chapter 2: Literature Review and Theoretical Background}

\section{Grand Theft Auto}

Before playing GTA 5 for this thesis, my engagement with the series was minimal. I did however play one of the first iterations of the game, before Rockstar transformed it into a juggernaut. This was Grand Theft Auto, a 2D game released in 1997 by DMA Designs. Viewed top-down, my best friend and I would inexpertly guide our pixelated avatar into nearby cars and run down pixelated pedestrians, turning them into pixelated jam-smears on the sidewalk. Though we were unaware of any controversy surrounding the game (and there was- even at its inception the game was criticized for hyper-violence), I do remember that there was a sort of transgressive thrill associated with starting up the boxy gray computer in the basement to play.

Overstating the importance of the GTA series in the zeitgeist of the gaming community is difficult. Describing the impact of a later title, GTA 3, Taylor writes that the game is both medium-defining and represents a profound shift in games studies. ${ }^{10}$ The subsequent games have shared this cultural importance. GTA 5 was developed by Rockstar North and distributed by Rockstar Games. It was released in September 2013 on the PlayStation 3 and Xbox 360, though during the course of my research I played a version released for $\mathrm{PC}$ in April 15. While it is the fifth numbered release, it is the fifteenth release in the series. GTA 5 was also the first title to have an online multi-player component, GTA Online, that continues to be updated with new content on a regular

\footnotetext{
10 Taylor, "From Stompin' Mushrooms to Bustin' Heads: Grand Theft Auto III as Paradigm Shift," 115; "GTA 5 Has SoldIn 85 Million Copies."
} 
basis. The game has sold 85 million copies since its release, approximately 10 million of which were in 2017, three years after its release, making it the best-selling game ever in the USA. ${ }^{11}$ This is to say nothing about the GTA games' contested status and role in changing regulation, censorship debates, and legal battles.

GTA 5 takes place in a fictionalized Los Angeles, California. Dubbed Los Santos, the city first appeared in a preceding title Grand Theft Auto: San Andreas. Within this updated and expanded gameworld, the narrative centers around three main characters, who the player can switch between at will, even within the same scene. Middle-aged Michael is deeply bored with the upper-class, poolside life he has retired to after "going straight". Franklin, a young black man, falls in with Michael as a way of moving up from low-level street crime to more glamourous and profitable white-collar crime. Finally, Trevor, dishevelled, disgusting, and dangerous, is Michael's old confederate, who now spends his days building a drug empire in the desert.

The main arc of the game's criminal exploits is a series of heists (although there is no shortage of supporting petty crime, car theft, assassinations). But narrative interest comes in part from the interweaving lives and relationships between the three men. Michael and Trevor have a long history, although until recently Trevor believed Michael to be dead. Franklin takes the role of go-between, peacemaker and surrogate son. Each man brings a cast of characters with him: hysterical wives, snivelling cronies, kooky aunts and troublemaking friends. The player also traverses their disparate geographical localities: from the South Side of Los Santos where we meet self-styled gangbangers; to

11 "GTA 5 Has Sold-In 85 Million Copies." 
the rural outskirts of the city populated by biker gangs, meth heads and trailer trash; to the glistening suburbs with households full of prescription-drug addled misery and ennui.

With dozens, if not hundreds, of hours of gameplay, it is nearly impossible to adequately summarize the game and its many complexities. What is more useful for my purposes is to situate the game within the context of the legal battles that centered on efforts to regulate violence in videogames, and to demonstrate how this is connected to debates and discussions in feminist movements and games studies about the risks of digital media and violence. The following section aims to provide a vocabulary for analyzing this game that brings together legal interventions, games studies, film studies and BDSM studies. Given that the GTA series has been a central figure in debates about the risks of violent video games as a medium, I pay particular attention to central theoretical debates about the nature of video games. I also provide a brief outline of feminist concern about the potential harms of transgression in digital media, and how this has been taken up by the courts in the United States in particular, where there had been considerable legal action taken against the developers of violent video games.

\section{Feminism, violence and harm in pornography and video games}

In 2005, Democratic Senators Hilary Clinton and Joe Lieberman tabled The Family Entertainment Protection Act, designed to impose heavy fines on video game vendors who sold games rated Mature (M) or Adult Only $(\mathrm{AO})$ to people under 17, among other regulations. In a press conference about the proposed legislation, Clinton describes the 
targeted games as violent and sexually explicit, with "deeply disturbing content". ${ }^{12}$ While the Senator conceded that games can be entertaining and educational, her concern rested with violent and pornographic games that she described as "stealing the innocence" of children by having them "commit gruesome acts [...] using top of the line graphics in stunningly realistic detail" ${ }^{13}$ The bill is not, Clinton assures us, a government censorship regime, but rather an attempt to regulate a potentially harmful product in the same way that we address other controlled products such as alcohol, tobacco and pornography. ${ }^{14}$ Her assumption that games, like porn, are somehow different and more potently dangerous than other media is clear. The tenor of this statement is remarkably similar to the pro-censorship, anti-pornography political advocacy that was underpinned by radical feminist understandings of media effects and harm.

Conversely, UNESCO's Mahatma Gandhi Institute of Education for Peace (UNESCO-MGIEP) have commissioned Paul Darvasi from the Royal St. George's College in Toronto to do studies on the potential of "serious" games that focus on "positive relationships rather than conflict" to engender empathy in players. ${ }^{15}$ Serious games are generally defined as video games designed for a purpose "higher" than pure entertainment. The term also describes games that are produced as teaching or learning tools for industries such as education. UNESCO-MGIEP director Anantha Duraiappah saw the advantages of serious games, asserting that "video games have an edge over

\footnotetext{
12 Tassi, "Can We Forgive Hillary Clinton For Her Past War On Video Games?"

13 FutureMajority, Hillary Clinton Hates On Video Games.

${ }^{14}$ FutureMajority.

15 "Are Video Games the Key to World Peace?"
} 
traditional classroom teaching" ${ }^{16}$ These games are often positioned as the anti-Grand Theft Auto, "more than mindless clicking", and potentially important enough to "save the world" ${ }^{17}$ But as I will discuss in Chapter 6, the idea that games are uniquely good teachers also feeds into essentializing readings of violence in games. As with pornography, games are understood as especially powerful. This places emphasis on the medium rather than the viewer/player and their engagement and experience. Davarsi does acknowledge that serious games won't automatically make people more empathetic, in the same way that violent games don't automatically turn people into gun-toting killers. ${ }^{18}$ However, both his narrative and Clinton's reinforce a constructed division between "good" and "bad" media, and invest in the belief that the media changes the way we think about and interact with the world, and this must have either pro-social or anti-social effects.

This division has a history. The deep schism within feminism triggered by differing perspectives on porn also coalesced around two opposing and often simplistic understandings of how media, violence, and eroticism come together. On one hand, porn was bad, degrading the rights and equality of all women because it normalized violence, socialized the conditions necessary for continued oppression, taught inequality, and exploited women and children. Alternatively, it was good because it subverted heteronormativity and monogamy, showed pleasure for pleasure's sake and expanded

\footnotetext{
16 "Are Video Games the Key to World Peace?"

17 Shapiro, "How Video Games Can Save The World"; McGonigal, Gaming Can Make a Better World; McGonigal and OverDrive, Reality Is Broken.

18 "Are Video Games the Key to World Peace?"
} 
sexual horizons to include queer possibilities. ${ }^{19}$ As I have attempted to show in the examples above, this diametric rhetoric continues to frame how we talk about new media. This binary is a gateway for state regulation of media considered to be obscene or as presenting too great a risk of harm. In the case of pornography, radical feminists had success in shifting the discourse, even if they were unsuccessful in having the legal decision makers completely adopt their anti-pornography views. They none-the-less opened the door for a host of legal instruments that justified controlling sexual expression on the basis of protecting society from risk. ${ }^{20}$ Unfortunately these laws have disproportionately impacted LGBTQ people and BDSM practitioners, highlighting the underlying morality of the legal interventions. ${ }^{21}$

In the following section, I aim to provide an overview of the culmination of the legal effort to regulate violent video games in the United States. Though ultimately unsuccessful, it is illustrative of how radical feminist rhetoric and strategy developed during the sex wars were applied in the freshly contested arena of video games. I then provide a brief analysis of the interdependence of media-effects research and radical feminist understandings of harm, in order to show how these arguments are mobilized not only in the court room, but also in public debates.

\section{A. The law and violence in videogames}

\footnotetext{
${ }^{19}$ Lacombe, Blue Politics Pornography and the Law in the Age of Feminism, 31; Dymock, "But Femsub Is Broken Too! On the Normalisation of BDSM and the Problem of Pleasure."

20 Lacombe, Blue Politics Pornography and the Law in the Age of Feminism, 37.

${ }^{21}$ Cossman, "Cossman, Brenda. 'Disciplining the Unruly: Sexual Outlaws, Little Sisters and the Legacy of Butler.' U. Brit. Colum. L. Rev. 36 (2003): 77. Harvard."
} 
Until the Supreme Court of the United States decided Brown v. Entertainment Merchants Association in 2011, there had been persistent efforts in the United States to legislate violent content in videogames. Many of these efforts involved connecting violence in media to the legal definition of obscenity (which always necessitates a sexual element). Therefore, while on its face Brown is about the First Amendment, not obscenity, the approach taken by anti-violence, pro-censorship advocates has meant that the decision rests entirely on the history of obscenity decisions. ${ }^{22}$

In the United States, the government's ability to restrict expression normally protected under the First Amendment is subject to strict scrutiny, making such impositions nearly impossible. ${ }^{23}$ However, material deemed obscene is seen to "stand outside freedom of speech" and is not subject to the same degree of scrutiny. ${ }^{24}$ The conditions for the classification of obscene material were set out in Miller v. California. In this decision, the SCOTUS set out a three-part test to use when determining whether material should be considered obscene. In such an instance, the courts should ask:

(a) Whether the average person, applying contemporary community standards, would find that the work, taken as a whole, appeals to the prurient interest; (b) whether the work depicts or describes, in a patently offensive way, sexual conduct specifically defined by the applicable state law; and (c) whether the work, taken as a whole, lacks serious literary, artistic, political, or scientific value. ${ }^{25}$

Those familiar with Canada's obscenity laws will be familiar with this archetype.

The Supreme Court of Canada (SCC) outlined a similar three-part test in $R v$. Butler. ${ }^{26}$

\footnotetext{
22 Post, "Post, David G. 'Sex, Lies, and Videogames: Brown v. Entertainment Merchants Association.' Cato Sup. Ct. Rev. (2010): 27.," 27.

23 Post, 29.

24 Post, 27.

25 Miller v California, 413 U.S. 15 (1973)

${ }^{26}$ R. v. Butler, [1992] 1 S.C.R. 452
} 
However, unlike the community imagined by the SCC, SCOTUS envisioned multiple communities existing simultaneously. ${ }^{27}$ This arouse from Ginsberg v. New York, a case centered around the owner of a luncheonette who had sold "girlie magazines" to a 16year-old boy. To analyze this fact scenario, the court employed the concept of "variable obscenity", to address material that is not obscene, but is nonetheless not suitable for young people. ${ }^{28}$ The decision rendered such material obscene as it relates to minors. In other words, material that is protected as free speech when accessed by adults may not have the same constitutional protections if it is distributed to children, or children are the intended audience. ${ }^{29}$ This has resulted in an understanding of obscenity which can fluctuate depending on the intended audience; it became permissible for the state to restrict minor's access to certain materials, such as commonplace softcore porn, because it is obscene as to minors. ${ }^{30}$

The statute in question in Brown v Entertainment Merchants uses Ginsberg in an effort to circumvent strict scrutiny and argue that violent videogames can be considered obscene as to minors, and subject to variable obscenity. The act aims to cover games in which:

... the range of options available to a player includes killing, maiming, dismembering, or sexually assaulting an image of a human being, if those acts are depicted" in a manner that "[a] reasonable person, considering the game as a whole, would find appeals to a deviant or morbid interest of minors," that is "patently offensive to prevailing standards in the community as to what is

\footnotetext{
27 Post, "Post, David G. 'Sex, Lies, and Videogames: Brown v. Entertainment Merchants Association.' Cato Sup. Ct. Rev. (2010): 27.," 32.

28 Post, 33, 34.

${ }^{29}$ Ginsberg v. New York, 390 U.S. 629 (1968)

30 Post, "Post, David G. 'Sex, Lies, and Videogames: Brown v. Entertainment Merchants Association.' Cato Sup. Ct. Rev. (2010): 27.," 35.
} 
suitable for minors," and that "causes the game, as a whole, to lack serious literary, artistic, political, or scientific value for minors." 31

In effect, the legislation aimed to piggyback restrictions on depictions of violence with restrictions focused on the sexual interests of minors and create a new category of expression exempted from First Amendment protection.

In the SCOTUS decision, the majority were unconvinced by this strategy, arguing that Ginsberg "is not a shield from heightened First Amendment scrutiny any time a legislature deems speech harmful to minors", and reinforced previous decisions which stated that new categories of unprotected speech cannot be added simply because they are deemed "too harmful to be tolerated". ${ }^{32}$ In the majority decision, the Court doublesdown on the importance of the sexual nature of obscenity. They reject the act's attempt to equate regulation addressing violence to obscenity regulations and write that "our cases have been clear that the obscenity exception to the First Amendment does not cover whatever a legislature finds shocking, but only depictions of "sexual conduct"". 33 The Court effectively quashed all of the legal paths that would have made it possible to link non-sexual violence with obscenity, and to therefore restrict children and youth's access to it. However, I believe there is still value in exploring how the efforts to connect the harm of non-sexual violence with obscenity persist in the public discourse, and especially in feminist anti-violent videogame advocacy. An understanding of the legal path can undergird an understanding of the ways pro-censorship arguments that were

\footnotetext{
31 Brown v. Entertainment Merchants Association, 564 U.S. 786 (2011) quoted in Post, 36. 32 Post, 40.

33 Brown v. Entertainment Merchants Association, 564 U.S. 786 (2011) quoted in Post, 40.
} 
effective in the regulation of pornography have been remobilized in the debate about violence and videogames.

Examining how the pro-censorship argument was constructed shows us several things. First, by rejecting the expansion in the definition of the obscene, the SCOTUS has effectively re-entrenched sex exceptionalism. ${ }^{34}$ By recognizing young people's first amendment rights in connection to violent material, while simultaneously upholding censorship that prevents teenagers from accessing sexually explicit material, the decision reaffirms the status of sex as exceptionally risky. The apparent paradox is not lost on the court itself; in his dissent, Justice Breyer writes:

... what sense does it make to forbid selling to a 13-year-old boy a magazine with an image of a nude woman, while protecting a sale to that 13-year-old of an interactive video game in which he actively, but virtually, binds and gags the woman, then tortures and kills her? What kind of First Amendment would permit the government to protect children by restricting sales of that extremely violent video game only when the woman - bound, gagged, tortured, and killed -is also topless? ${ }^{35}$

Second, the effort to show how violence might appeal "to the deviant or morbid interests" of young people, allows us to see how this conceptualization of harm is linked to transgression, and therefore to eroticism. There appears to be an acknowledgement of the inherent appeal of violence within the statute, and the fear here seems to be that video games have the potential to allow us to access these deviant and morbid desires in a pleasurable sense and in a way that goes against cultural standards, or established taboos.

\footnotetext{
34 Khan, "Hot For Kink, Bothered by The Law: BDSM and the Right to Autonomy."

${ }^{35}$ Brown v. Entertainment Merchants Association, 564 U.S. 786 (2011)
} 
This is akin to Helen Hester's repurposing of the concept of prurience to describe non-sexual pornography. While the courts have rejected this connection, the similarities between violent transgression and sexuality are practically taken for granted by antiviolence advocates, whose concern points towards and seems to confirm the pleasurable potential of transgression and violence. Hester's work is concerned with a broader conceptualization of porn which includes representations of disgust, images of violated bodies, and autobiographical accounts of trauma which have only a spurious link to adult entertainment (or what we would conventionally call porn). ${ }^{36}$ Her book, Beyond Explicit is an attempt to think through the displacement of sex in the contemporary understanding of the pornographic and the "explosion" of the pornographic as a cultural concept. ${ }^{37}$ Considering this, we might argue that the efforts to censor violence in video games using the formula of obscenity law is an implicit assertion of the affective similarities between violence and sexuality. Understanding this connection through the framework of transgression and eroticism seems productive, and I will elaborate on it in Chapter 4.

However, in the following sections I want to describe some more tangible similarities between approaches to pornography and to violent video games. Advocacy for regulation of both products relies heavily on media-effects research, which in turn impacts radical feminist understandings of the harms these media may generate.

\section{B. Media-effects models of porn and violent videogames}

\footnotetext{
36 Hester, Beyond Explicit, 13.

37 Hester, 14.
} 
The psychological literature in media effects theory is quite vast, and it is beyond the scope of my thesis to provide a detailed summary. Instead, I will provide a brief overview of the major theories that contribute to the debate, including the General Aggression Model (GAM), conceived by Craig A Anderson, as a way of acknowledging that this is the dominant framework of harm with which the law, mainstream media, and concerned parents engage with video game controversies. ${ }^{38}$ There are a number of models that aim to explain the relationship between video games and real-life violence or aggression. The GAM is one of the best known and it is representative of the others, most of which rely on social modeling theory. ${ }^{39}$ GAM proposes that aggressive behaviours are caused by "the learning, activation, and application of aggression-related knowledge structures stored in memory". ${ }^{40}$ Drawing on the concept of social scripts, where each violent-media exposure acts as one more "learning trial", the model proposes that knowledge structures that support and reinforce violent or aggressive behaviors are rehearsed and cemented in gameplay, ultimately leading to an increase in aggressive personality. ${ }^{41}$ Researchers use this model to show that playing violent games will increase a person's aggression in the short and long term because games "teach" the player aggression, by "priming" their brains for aggressive responses to social

\footnotetext{
${ }^{38}$ Anderson and Bushman, "Effects of Violent Video Games on Aggressive Behavior, Aggressive Cognition, Aggressive Affect, Physiological Arousal, and Prosocial Behavior," 355.

39 Ferguson, "Evidence for Publication Bias in Video Game Violence Effects Literature."

${ }^{40}$ Anderson and Bushman, "Effects of Violent Video Games on Aggressive Behavior, Aggressive Cognition, Aggressive Affect, Physiological Arousal, and Prosocial Behavior." 355

41 Ibid. 355; Matthew S. Eastin, "Video Game Violence and the Female Game Player: Self- and Opponent Gender Effects on Presence and Aggressive Thoughts," Human Communication Research 32, no. 3 (July 2006): 351-72, doi:10.1111/j.1468-2958.2006.00279.x. 351
} 
situations. ${ }^{42}$ As this process is repeated and players engage more and more with violence, researchers suggest that they will be more likely to interpret everyday situations as malicious, more likely to make impulsive rather than thoughtful decisions, and ultimately more likely to believe that aggression is an appropriate response. ${ }^{43}$

Other behavioural psychologists focus on the process of desensitization to violence. These researchers argue that video games have become "more graphic, realistic and violent over time". ${ }^{44}$ Desensitization theory proposes that exposure to violent video games reduces the negative responses to real life violence, including reducing empathetic response for the pain and suffering of others, and reducing aggressive inhibitions and aversion to violence. ${ }^{45}$ Players may also experience increased desire for violence, combined with desensitization, causing them to escalate their behaviours.

Researchers generally argue that the more graphic the media, the greater the desire for aggression or violence. ${ }^{46}$ Kevin Runions, for example, asserts that using either porn or violent games may be an expression of "problematic sensation seeking", linked to appetitive types of aggression (as opposed to situational manifestations as explained above). ${ }^{47}$ This psychological research is intended to scientifically describe cause and effect relationships, and neatly delineate effects and correlations. ${ }^{48}$

\footnotetext{
42 Anderson and Bushman, "Effects of Violent Video Games on Aggressive Behavior, Aggressive Cognition, Aggressive Affect, Physiological Arousal, and Prosocial Behavior." 355

${ }^{43}$ Adachi and Willoughby, "The Effect of Violent Video Games on Aggression." 56

44 Engelhardt et al., "This Is Your Brain on Violent Video Games," 1033.

45 Engelhardt et al., "This Is Your Brain on Violent Video Games."

${ }^{46}$ Allen, D'Alessio, and Brezgel, "A Meta-Analysis Summarizing the Effects of Pornography II Aggression After Exposure."

${ }^{47}$ Runions, "Toward a Conceptual Model of Motive and Self-Control in Cyber-Aggression."

${ }^{48}$ Barker, "Psychology and Pornography," 122.
} 
While researchers who argue that video games result in real life violence tend to capture the imagination of the media, there is an opposing group of behavioural psychologists who reject this cause and effect connection. Criticisms within the discipline are largely focused on experimental design. These criticisms include that the studies fail to match games being used in the experiments on other variables such as competitiveness, difficulty and pace of action and that because these variables are unaccounted for they may be responsible for increases in aggression or arousal. Researchers also argue that participants don't play games for long enough to become competent, making it possible that any increase in aggression is caused by the frustration of attempting to master complex controls and visual-spatial navigation. The test used to measure aggressiveness is also disparaged because it has never been correlated with trait aggression, domestic violence, or violent criminal acts. ${ }^{49}$ Even simple things like definitions of violence or aggression may not be consistent across studies. ${ }^{50}$ Combined with the lack of evidence from natural settings, whether such findings are generalizable is highly contested. ${ }^{51}$

The views of the judiciary tend to align with critics of the psychological studies professing to show evidence of a link between playing violent games and aggressive or violent behavior. Justice Scalia, writing for the majority in Brown v. Entertainment Merchants Association, concurred with lower courts that had looked at the psychological

\footnotetext{
${ }^{49}$ Ferguson, "Evidence for Publication Bias in Video Game Violence Effects Literature"; Ferguson and Kilburn, "The Public Health Risks of Media Violence"; Adachi and Willoughby, "The Effect of Violent Video Games on Aggression"; Ferguson and Rueda, "The Hitman Study."

50 Barker, "Psychology and Pornography," 121.

51 Barker, 121.
} 
evidence. Specifically addressing the research done by Anderson, he writes that "These studies have been rejected by every court to consider them, and with good reason: they do not prove that violent video games cause minors to act aggressively $[\ldots . .]^{\prime \prime}{ }^{52}$ Like many critics of Anderson and his ilk, Scalia argues that the evidence is correlational, not causational, and that the studies have "significant, admitted flaws in methodology". 53

Nonetheless, in his dissent, Justice Breyer provides two lengthy appendices regarding whether or not playing violent video games poses psychological risk. Through introducing these, he aims to suggest that there is "substantial (though controverted) evidence supporting [... the conclusion] that violent video games can cause children psychological harm", especially when compared to "more passive media" (books, movies, etc.). ${ }^{54}$ Justice Breyer therefore keeps the psychological research in play and provides fuel for the fire of any social scientist or advocate who sees games and interactivity as particularly insidious. Interestingly, the central role of psychology in public discourse about violent video games has persisted, even as majority decision in Brown appears to quash its utility as the backbone of a pro-censorship argument.

\section{Feminism and Harm}

The Sex Wars were a period of intense debate between feminists of various factions and other political groups about pornography, sadomasochism, and sexuality more broadly. The peak of these debates was during the 1970s to the 1990s, although there was no

\footnotetext{
52 Brown v. Entertainment Merchants Association, 564 U.S. 786 (2011)

53 Brown v. Entertainment Merchants Association, 564 U.S.

54 Brown v. Entertainment Merchants Association, 564 U.S. 786 (2011)
} 
clear resolution, and as I will argue, the debates of the Sex Wars has shifted and been taken up to address pornographic media including violent or transgressive video games. ${ }^{55}$

During this period, both large schisms and smaller rifts were created as a result of the pornography debates. While the finer points of these distinctions are beyond the scope of this project, I will broadly outline the three major (and often internally diverse) feminist positions, summarized in the table below. For a full discussion of the Sex Wars, see Lacombe (1998) and Khan (2014). ${ }^{56}$

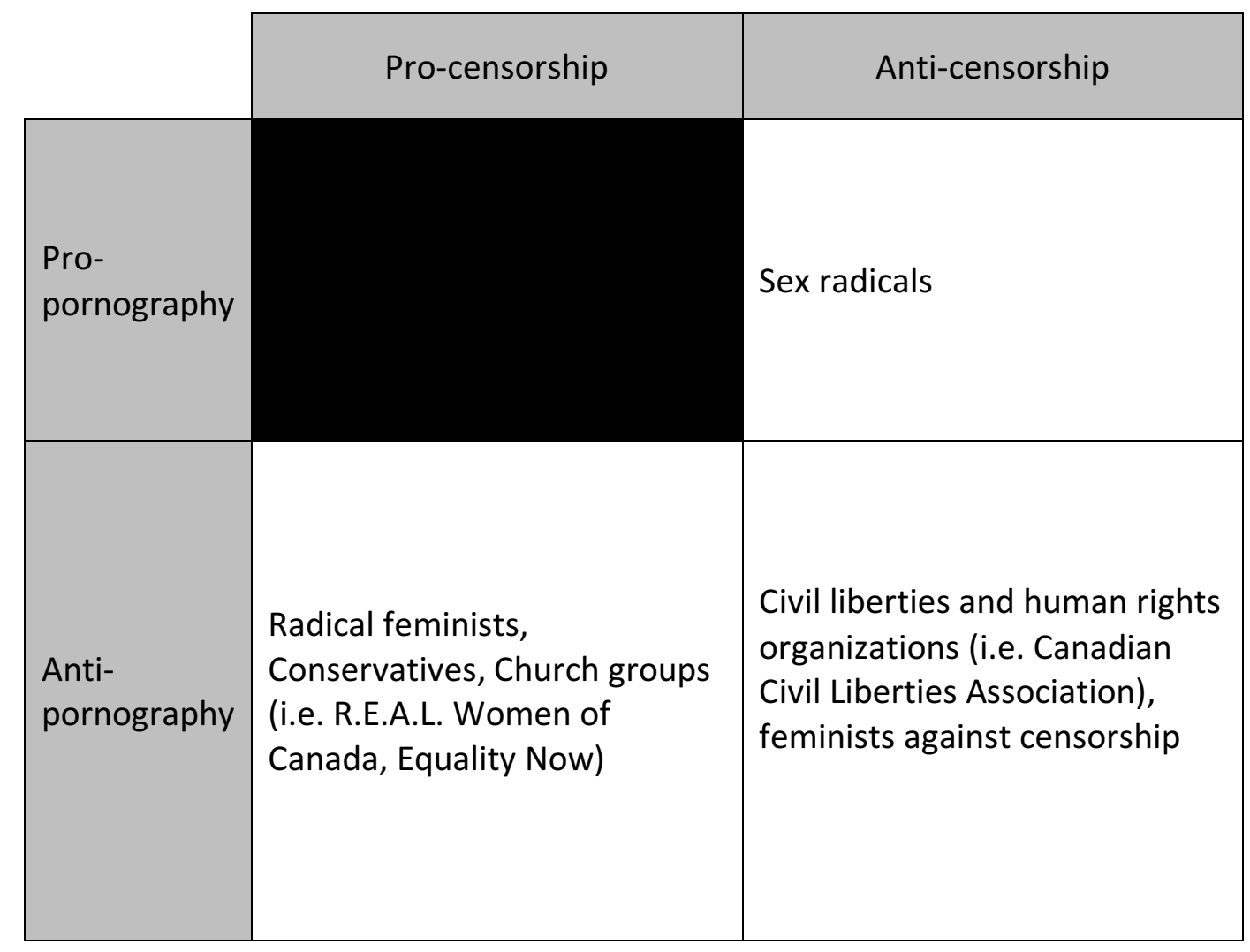

55 Khan, Vicarious Kinks, 54.

56 Khan, Vicarious Kinks; Lacombe, Blue Politics Pornography and the Law in the Age of Feminism. 
During the Sex Wars, a focus on harm brought together radical feminist and conservative organizations in a "precarious alliance of anti-pornography, pro-censorship" belief. ${ }^{57}$ For these groups, not only porn but any explicit sexual material (including erotica) harms society and the individual and should therefore be regulated and censored. ${ }^{58} \mathrm{~A}$ modern correlate to this within the violent video game debate exists in the efforts by the radical feminist organization Equality Now to regulate the sale of games featuring rape in Japan, or the efforts by conservative lawmakers discussed above to censor and regulate violent video games. ${ }^{59}$ While these two groups would ordinarily have little in common, they are united as unlikely bedfellows through their belief that "pornographic" texts are inherently harmful, and that this can be supported with science.

On the other hand, civil liberties and human rights organizations tended to agree with the anti-pornography stance taken by radical feminists that pornography "objectified, degraded and exploited" women and children, but disagreed that pornography should be censored, arguing that science did not conclusively prove that they were harmful. ${ }^{60}$ While they recognized that "pornography, like any other text, influences the reader" they were unwilling to push for censorship, arguing that "its effects are not harmful enough to warrant the use of the criminal law" ${ }^{61}$

Linked to this position is that of the feminists against censorship. While these groups were often dedicated to anti-pornography, they disagreed with the civil

\footnotetext{
57 Lacombe, Blue Politics Pornography and the Law in the Age of Feminism, 47.

58 Lacombe, 49.

59 Equality Now, "Japan: Rape Simulator Games and the Normalization of Sexual Violence"; Galbraith, "RapeLay and the Return of the Sex Wars in Japan."

60 Lacombe, Blue Politics Pornography and the Law in the Age of Feminism, 54.

61 Lacombe, 55.
} 
libertarians' approach to anti-censorship. Instead, they critiqued the latter's dependence on freedom of expression, arguing that it failed to take into account the "structures that oppressed and silenced women and minorities" and the unequal way censorship would impact these groups. ${ }^{62}$ Similarly, while they often agreed that pornography was problematic and that is was, at its core, "woman hating", they also disagreed with the radical feminist position that "pornography reveals men's true sexuality- its latent sadism". ${ }^{63}$ Instead, they refocus on the social construction of sexuality, as well as its "cultural and historical specificities". ${ }^{64}$ Feminists against censorship rejected essentializing understandings of textual meaning, arguing that the production of meaning is complicated and "arises from the structural context in which images or works find themselves". 65

Feminists against censorship understood "pornography as a way of seeing, rather than as the truth of sex". ${ }^{66}$ We can see the continuation of such an approach with the Tropes vs Women in Video Games series, produced by Anita Sarkeesian, which aims to teach viewers this way of seeing. ${ }^{67}$ Like anti-pornography, anti-censorship feminists, Sarkeesian rejects the idea that men are inherently violent, and instead seeks to read video games, like pornography, for "specific devices that make it a genre" ${ }^{68}$ Her work seeks to understand the depiction of women in video games using the language of

\footnotetext{
62 Lacombe, 56.

63 Lacombe, Blue Politics Pornography and the Law in the Age of Feminism.

64 Lacombe, 57, 59.

65 Lacombe, 57.

66 Lacombe, Blue Politics Pornography and the Law in the Age of Feminism, 58.

67 Sarkeesian has gained a substantial audience through her video series Tropes vs. Women in Video Games online video series, which raised 160,000 USD in funding via Kickstarter.

68 Lacombe, Blue Politics Pornography and the Law in the Age of Feminism.
} 
pornography. Given the intellectual history of anti-pornography, anti-censorship feminists, this is not surprising. As Valverde, explains, these feminists understood pornography in a way that sought to identify and authoritatively read sexism in many forms of media:

Rather than try to isolate porn as a particular object, it might be more useful to speak about "the pornographic" as an aspect of many apparently harmless films, books, and magazines. When we stop worrying about defining porn narrowly enough so that only the worst stuff comes under legal scrutiny and begin to define it broadly enough to encompass those aspects of mass culture that glamourize the subjection of women, then we will have learned something not just about male lust but also about women's own fantasies and desires. ${ }^{69}$

Sarkeesian's analysis is a natural extension of this approach, which often sought to demonstrate that pornographic devices appeared outside of sexually explicit material, and that other cultural forms often exploited pornographic themes and codes. ${ }^{70}$ Like feminists against censorship, Sarkeesian doesn't see censorship as the solution to sexism in games, she is more inclined to advocate for positive imagery and women-made games.

However, as Lacombe points out, while feminists against censorship often try to contextualize images in order to distinguish themselves from radical feminist approaches, they often foreground their own interpretations in such a way that they "over determines all other contexts". ${ }^{71}$ Lacombe uses the example of Valverde's reading of a woman performing oral sex as being about subordination (as opposed to a man performing cunnilingus, which is about pleasure). The context actually seems to matter

\footnotetext{
69 Valverde, Sex, Power, and Pleasure, 123.

70 Lacombe, Blue Politics Pornography and the Law in the Age of Feminism, 58; Valverde, Sex, Power, and Pleasure.

${ }^{71}$ Lacombe, Blue Politics Pornography and the Law in the Age of Feminism.
} 
little here. As Lacombe points out, Valverde's reading of this generic fellatio scene seems to suggest that its meaning is fixed in time and space, in a "social system of female subordination and male domination", and that this system determines the meaning of the image regardless of other social systems or individual contexts of the viewer. ${ }^{72}$ Similarly, while rejecting the idea that there is a "one-to-one cause and effect" between image and action, Sarkeesian often takes certain signifiers (the existence of in-game sex workers, for example) to be indicative of the inescapable context of sexism in gaming. ${ }^{73}$ As with Valderde, sexism is the prime context, "so powerful and encompassing that it determines pornography and our reaction to it" ${ }^{74}$

Lacombe also distinguishes a third group that she calls sex radicals. These feminists, though diverse, were generally pro-pornography and anti-censorship. Embracing pluralism and rejecting normative conceptualization of sexuality and feminist distortions of pleasure into false consciousness, sex radicals recognize the audience' (and especially women's) agency and interpretative power. As Lacombe explains: "the recognition that women respond positively to images created for men's pleasure made it imperative for sex radicals to examine the elements other than sexism, at work in pornography and to investigate how women appropriate pornographic images". ${ }^{75}$

\footnotetext{
72 Lacombe, 61.

73 Sarkeesian, Women as Background Decoration (Part 1).

${ }^{74}$ Lacombe, Blue Politics Pornography and the Law in the Age of Feminism, 63.

75 Lacombe, 66.
} 


\section{Radical Feminism and Harm}

During the Sex Wars, radical feminists were quick to rely on scientific evidence as soon as it seemed to corroborate their claims. They drew on the work of social psychologists like Donnerstein and Malamuth, who purported to show that porn presented a 'substantial risk' to society. ${ }^{76}$ Experimental psychology continued to inform the feminist position through reports like the US Meese Report on pornography, released in 1986. For both feminists and psychologists, the realism of porn, and now games, is often seen as a conduit for violence, that primes and trains viewers/players for aggressive responses within the real world. ${ }^{77}$ In both cases, social science, and particularly psychological research provided a powerful but contentious backdrop to legal debates. ${ }^{78}$ In their argument about the power of politicians, advocacy groups, and health science research in censorship crusades, Day and Hall draw on the concept of the Werther effect. Coined by David Phillips, and in reference to the belief that young men in Europe were killing themselves to emulate the protagonist in The Sorrows of Young Werther after its publication in 1774 , the term describes the phenomenon of "copycat" behaviour resulting from fiction or news coverage. ${ }^{79}$ The authors argue that throughout

\footnotetext{
76 Lacombe, 34.

77 Anderson and Bushman, "Effects of Violent Video Games on Aggressive Behavior, Aggressive Cognition, Aggressive Affect, Physiological Arousal, and Prosocial Behavior."

${ }^{78}$ Byrd, "It's All Fun and Games Until Someone Gets Hurt: The Effectiveness of Proposed Video-Game Legislation on Reducing Violence in Children"; Phillips, "Virtual Violence or Virtual Apprenticeship: Justification for the Recognition of a Violent Video Game Exception to the Scope of First Amendment Rights of Minors." Ind. L. Rev. 36 (2003): 1385."; Hall, Day, and Hall, "A Plea for Caution."

79 Day and Hall, "Déjà Vu: From Comic Books to Video Games: Legislative Reliance on’Soft Science'to Protect Against Uncertain Societal Harm Linked to Violence v. The First Amendment," 421.
} 
history, the social sciences have supported legal efforts to prohibit or regulate anything perceived to degrade social morality or cause harm. ${ }^{80}$ The anti-violent video game rhetoric mirrors that of anti-pornography feminists, particularly in the way both attempt to mobilize the protectionist inclinations of the courts using scientifically supported conceptualizations of harm, is yet another example of this.

In Canada, feminist activists mobilized social science in their favour by linking pornography with three kinds of harm. First, they argued that there was a direct harm to the women involved in making pornography. Second, they argue that there was an indirect harm at the level of consumption: watching pornography would increase violence against women. ${ }^{81}$ Third, there is a broad social harm. Pornography harms men and women by forcing them into rigid gender roles: trapping women in passivity and limiting men's full range of emotional possibility. ${ }^{82}$ In fleshing out these ideas, feminists relied heavily on scientific evidence, in part because it was helpful in counteracting libertarians' demands for clear-cut proof for the harmful effects of porn. ${ }^{83}$ As in current discussions about video games and harm, feminist advocates brandished studies purporting to show harm with far more certainty than even the study's writers. ${ }^{84}$ In this context, "ambiguities and uncertainties" are ignored and reframed as evidence that can be mobilized and construed into a call for action. ${ }^{85}$

\footnotetext{
80 Day and Hall, 422.

81 Lacombe, Blue Politics Pornography and the Law in the Age of Feminism, 31.

82 Lacombe, 32.

83 Lacombe, 31, 34.

${ }^{84}$ Lacombe, 36.

${ }^{85}$ Lacombe, 36-37.
} 
In discourse about video games, harm is often understood in much the same way, (with the exception of the first kind of direct harm- it is currently difficult to argue that direct harm is occurring, because most characters within the game are wholly digital, although this may change as more actors are used in motion-capture). The indirect harm of videogames is said to be the impact it has on the aggressive and violent tendencies of players. Psychological research ardently argues that video games are changing behaviour on an individual level, which results in greater risk of interpersonal violence. ${ }^{86}$ Other researchers have outlined the ways in which video games produce a broader form of societal harm. Their argument supposes that videogames, like pornography, reinforce "sex role stereotypes" or other forms of social stereotyping based on age, race, and gender. ${ }^{87}$ These researchers also share a particular concern about the mixing of violence and sex (what Dill calls eroticized aggression). ${ }^{88}$

Sarkeesian is an excellent example of the way feminist discourse continues to mobilize evidence rooted in psychology and media effects research. Sarkeesian has taken up an important role in game criticism and has faced an enormous and often violent backlash because of it. Her work highlighting violence and harassment in gaming culture is particularly valuable. However, Sarkeesian's analysis of the content of games often relies on a simplistic understanding of harm that harkens back to anti-pornography radical feminism. Her understanding of harm concerns both indirect but interpersonal

\footnotetext{
${ }^{86}$ Anderson and Bushman, "Effects of Violent Video Games on Aggressive Behavior, Aggressive Cognition, Aggressive Affect, Physiological Arousal, and Prosocial Behavior"; Adachi and Willoughby, "The Effect of Violent Video Games on Aggression"; Engelhardt et al., "This Is Your Brain on Violent Video Games."

87 Dill et al., "Violence, Sex, Race and Age in Popular Video Games: A Content Analysis."

88 Dill and Thill, "Video Game Characters and the Socialization of Gender Roles," 859.
} 
harm and broad societal harm. In one video she explains that while video games "won't turn players into raging sexists [...] we don't have a 'monkey see, monkey do' cause and effect relationship with the media we consume", her critique also rests on the idea that violent media, as a form of centralized storytelling, have a "powerful cultivation effect" that shapes cultural attitudes and opinions, reinforces the dominant gender paradigm, and negatively effects feelings towards real-world women. ${ }^{89}$ Cultivation theory does purposefully deviate from media effects research's focus on immediate behavioural or attitudinal changes in those exposed to violent media. Instead, it focuses on the implication of the accumulation of messages, images and values. ${ }^{90}$ However, cultivation theory still has a basic underlying assumption that the messages being transmitted is stable, and that while an individual's race, gender, age or other demographic feature may make a difference in how that message is integrated into their lives, the power of television (for Gerbner) or games (for Sarkeesian) is ultimately inescapable and homogenizing. ${ }^{91}$

Sarkeesian treats interactivity as the key principal issue in understanding harm, arguing that because of their interactivity, players take on a "unique and more detrimental" position as participants in the in-game violence, as compared to the audience of a film or book who are mere voyeurs. ${ }^{92}$ The result is that players "collaborat[e] with developers" in the sexual objectification of, and violence against,

89 "Damsel in Distress (Part 2) Tropes vs Women"; Sarkeesian, Women as Background Decoration (Part 1).

90 Gerbner et al., "Living with Television: The Dynamics of the Cultivation Process," 21.

91 Gerbner et al., 24.

92 Sarkeesian, Women as Background Decoration (Part 1). 
women. ${ }^{93}$ It is interesting how these concerns transition relatively smoothly between porn and videogames. Whereas porn is often thought of as particularly harmful because of its connection to sex and sexuality (in the most basic terms, the images are connected to the real-world act of masturbation), the connection between controller and action makes games particularly dangerous. In both cases, it is the "joystick", or the connection between what is perceived and what is felt, that is the source of both the concern, and the pleasure. I will return to these conceptualizations of harm in Chapter 6 where I discuss race play. In that section, I engage with critical race analyses that argues video games may cause harm by reinforcing racial stereotypes and consider other possible reparative readings

\section{A Vocabulary for Talking About Video Games}

A. Games as unique vs. particular

Among both lay people and some game studies researchers, there is a common assumption that videogames are unique mediums of experience. As game critic Brendan Keogh argues, concepts associated with videogames like "immersion, gameplay, interactivity, [and] re-playability" are used to argue that there is a unique experience associated with this leisure activity. But Keogh cautions that these concepts are also laden with assumptions that undermine attempts to understand the affective potential of videogames. He argues that the tendency to hyper-focus on interactivity and immersion, alongside the presumption that other media forms lack interactivity, divorces games

\footnotetext{
93 Sarkeesian.
} 
analysis from the long history of media criticism. To counterbalance this tendency, Keogh offers a mantra: "videogames aren't special, and they aren't unique". ${ }^{94}$ Instead, he suggests we should think of video games as particular, which he believes to be a more cautious and less celebratory assertion. ${ }^{95}$

When we isolate a form or medium as special (or especially dangerous) there is a tendency to decontextualize them. Steve Jones draws attention to this phenomenon in his discussion of 'extreme' porn. He argues that overstating the transgressiveness of extreme porn paradoxically tends to diminish its meaning. Similarly, the hyperbolic focus on interactive violence in games isolates games from a media landscape in which violence and transgression is ubiquitous and erases nuance between degrees of extremity, homogenizing the material. ${ }^{96}$ Research on violent games often names titles like Grand Theft Auto, Doom, and Hitman in the same breath. Violence is certainly a core feature in these games, but their particularities in mechanics, narrative, character development, satire, music, graphics, engine, code, and hundreds of other vectors of meaning are obfuscated when they are labelled as simply 'exceptionally violent'. The result is that little effort is made to understand how players relate to these texts, and what these games say about our relationship to violence, death and transgression. ${ }^{97}$ Focusing on games' uniqueness rather than their particularity as a media form, feeds into Gamer Culture. I use -capital G- Gamer as opposed to gamer/player here because Gamer Culture is defined in part by its desire to tightly police its boundaries, to

\footnotetext{
${ }^{94}$ Keogh, "Videogames Aren't Special. Videogames Aren't Unique."

95 Keogh.

96 Jones, "'Extreme' Porn?," 302.

97 Jones, 302.
} 
assert its entitlement as the "guardian of games", and to provide authoritative interpretations regarding what is good and what is not. As Keogh argues, Gamers come to see themselves as powerful and more active than a "supposedly passive cinema audience tube-fed a movie".$^{98}$ If researchers treats games as exceptional, we contribute to propping up Gamers' (often tangibly harmful) desire to maintain their exclusive and special status. Given how analysis of video games, Gamer Culture, and the experiences of gamers/players intersect, placing games in their context as the latest in an incredibly long line of media advancements and art is critical.

The idea that games are unique is also aimed at setting them apart from other forms of media, such as films and books. As Keogh argues, videogames are different from other forms of media and art, but they are "structurally related" to each of these as well. While the differences between these forms of media is often emphasized in an effort to distinguish games and to defend the importance of engaging with them as art and subjects of scholarly interest, the similarities between them are equally important. Below, I will discuss two concepts often used to argue that games stand apart: the difference between ludology and narratology (or, the fact that videogames are games), and the role of immersion. While these are important debates that contextualize my arguments, I ultimately agree with Keogh that to consider videogames strictly as a "formal subset of "games"” doesn't allow us to understand the complex entanglements between body, experience, and game..$^{99}$

\footnotetext{
98 Keogh, "Videogames Aren't Special. Videogames Aren't Unique."

99 Keogh, A Play of Bodies, 10.
} 
Keogh both emphasizes the importance of the question "what sets videogames apart" and simultaneously decenters it in his own analysis. For him, videogames are above all an embodied phenomenon, and understanding the way players "use videogames with their bodies" is of preeminent importance in games studies. ${ }^{100}$ While we could argue that consumption of books and films also involve some degree of embodiment, videogames "always rely on" and incorporate the embodied player. ${ }^{101}$

Like film, videogames require that the player engage with "a screen and the space depicted "beyond" it" ${ }^{102}$ But they also go further: drawing on Sudnow, Keogh describes videogames as "microworlds: small worlds that we don't inhabit in any straightforward manner but that we engage with from a distance, our actions reflected back to us, translated by lights and colours and sound". ${ }^{103}$ Through gameplay, the self "seems to flicker between bodies and worlds", in what he calls a play of bodies, a concept I will return to in Chapter 5 when I discuss the cyborg self. ${ }^{104}$ In other words, while videogames require an audience that, like film audiences, is able to "look and listen", they are also required to physically "touch and move", which Keogh argues is an embodied and situated experience. Player and game are therefore meaningfully co-constituted in a "complex entanglement" between the body and the virtual world. ${ }^{105}$

\footnotetext{
100 Keogh, 4.

101 Keogh, 5.

102 Keogh, 9.

103 Keogh, 3.

104 Keogh, 3, 13.

105 Keogh, 10.
} 


\section{B. Ludology and Narratology}

Perhaps the most fraught and longstanding issue is that of ludology and narratology. At its most distilled, the debate centers on whether the analysis of games should focus primarily on narrative elements (often considered to be holdover strategies from other disciplines such as film studies) or ludology, which distinguishes itself primarily based on its focus on the rule systems and mechanics of a game. Frasca has stated that his use of the term ludology is meant to evoke a "yet non-existent discipline that would focus on the study of games in general and videogames in particular". ${ }^{106}$ Aarseth's use of the term links it to the understanding of games as ergodic, meaning it requires "nontrivial effort" in "traversing the text" ${ }^{107}$ This distinction is neatly summed up as a dissimilarity between a reader and a player:

[...T] he [videogame] user is basically involved as a player (doing ergodic work on the materiality of the text), not as a reader (interpreting on a semantic level). This may sound obvious (games are games), but it is an important theoretical premise if we are to avoid studying computer games as if they were just another narrative genre. $^{108}$

For an outsider, including myself, the vitriol contained within this debate can be confusing. It's perhaps most easily explained as growing pains in a newly emerging field of study attempting to account for the issue of play, and to distinguish itself from practically primordial arenas like literature studies. For example, Klevjar argues that

\footnotetext{
106 Frasca, "Ludologists Love Stories, Too: Notes from a Debate That Never Took Place."

107 Aarseth, Cybertext, 1.

108 Klevjar, "In Defense of Cutscenes," 192.
} 
because games studies is a newly developing field, "it is important not to leave it open to affirmative appropriation by established disciplines and theories"; and in the first edition of the journal Games Studies, editor-in-chief Espen Aarseth urges caution against the "colonizing attempts" of literature and film studies. ${ }^{109}$

I'm presenting this debate here, not because it is of central importance to my own study of games, but because it seems to cast a persistent shadow over games studies. And also, because it has informed the way we talk about violence in games as both a mechanic and a core part of narrative meaning. However, this schism seems to make less and less sense as scholars move towards increasingly interdisciplinary modes of study. For me, as someone attempting to work at the intersection of a handful of fields, the desire to carve out games as unique and games studies as an island seems limiting at best. At worst, it has resonated with a set of Gamers who claim dominion over games themselves. The claim that games studies is a "domain that belongs only to those who are studying games as abstract rule systems" is uncomfortably similar to that of the subset of Gamers who claim the exclusive right to interpret, understand and eng age meaningfully with games. ${ }^{110}$ Ultimately, ludologists are "debating a phantom of their own creation". Recognizing this, Janet Murray calls for game studies to be reframed as "not a zero-sum team contest, but a multi-dimensional, open-ended puzzle that we all are engaged in cooperatively solving." ${ }^{111}$ I will return to this in a roundabout way in Chapter 4 on transgression and dark play in order to illustrate how both the narrative and the

\footnotetext{
109 Klevjar, 192.

110 Murray, "The Last Word on Ludology v Narratology in Game Studies." In . 2005."

111 Murray.
} 
mechanics of a game are factors which a player must mediate and balance in order to experience playful tension.

\section{Immersion and identification}

Given that scholars have been trying to tease out the meaning and importance of games' interactivity for the past decade, it is somewhat cliché to state that immersion is an ambiguous and vague term. However, its frequent use by game critics and players to describe their gameplay experiences highlights both its importance to gamers and the importance of clarifying its meaning. While some scholars have attempted to objectively measure immersion, my use of the concept throughout this thesis will follow the research of scholars who are focused on the experiences and knowledge of the player, as well as the cultural and social factors that impact their play. ${ }^{112}$ Because of this, immersion is conceptualized as a subjective experience that cannot be quantitatively analyzed.

Brown and Cairns conducted a grounded study focused on understanding what players understood immersion to be in the context of video games. They found that players describe a range of experiences as immersive, and that these range in depth of engagement. ${ }^{113}$ These levels of engagement are 1) engagement, 2) engrossment and 3) total immersion. ${ }^{114}$ As the play experience becomes more immersive players must overcome more barriers to immersion, including include having access (the ability to understand and use the game's controls) or empathy (the ability to empathize with the

\footnotetext{
112 Jennett et al., "Measuring and Defining the Experience of Immersion in Games"; Ermi and Mäyrä, "Fundamental Components of the Gameplay Experience: Analysing Immersion," 9.

113 Brown and Cairns, "A Grounded Investigation of Game Immersion," 1297.

114 Brown and Cairns, 1297.
} 
character(s), as well as to invest more time, effort and attention. ${ }^{115}$ Brown and Cairns equate total immersion (the most immersive experiences) with presence, inasmuch as it generates feeling of being "cut off from reality, and detachment to such an extent that the game was all that mattered" ${ }^{116}$ However, the experience of total immersion is fleeting. ${ }^{117}$

While Brown and Cairns demonstrate that there are different degrees of immersion, Ermi and Mäyrä show that immersion can be provoked by different factors which may interact with one another. They put forward a heuristic gameplay experience model based on their work with game-playing children. Known as the SCI-model, it outlines three types of immersion: sensory, challenge-based and imaginative. ${ }^{118}$ Sensory immersion is concerned with the audiovisual nature of games. ${ }^{119}$ Challenge-based immersion is rooted in the ludology of the game (it's rules, mechanics, controls), and is at its most potent when there is a fulfilling balance between challenge and player ability. ${ }^{120}$ Finally, imaginative immersion involves the ways in which players may become absorbed into the narrative or world or the game, or begin to identity with the character(s). ${ }^{121}$

While these definitions are designed for utility in games research, the discussion of immersion provided by Janet Murray is enriching from a theoretical perspective. She writes:

\footnotetext{
115 Brown and Cairns, 1298, 1299.

116 Brown and Cairns, 1299.

117 Brown and Cairns, 1299, 1300.

118 Ermi and Mäyrä, "Fundamental Components of the Gameplay Experience: Analysing Immersion," 1.

${ }^{119}$ Ermi and Mäyrä, 7; For more on the role of sound in immersion in video games, see Grimshaw. Grimshaw, "Sound and Immersion in the First-Person Shooter."

120 Ermi and Mäyrä, "Fundamental Components of the Gameplay Experience: Analysing Immersion," 8.

${ }^{121}$ Ermi and Mäyrä, 8.
} 
We seek the same feeling from a psychologically immersive experience that we do from a plunge in the ocean or swimming pool: the sensation of being surrounded by a completely other reality, as different as water is from air, that takes over all of our attention, our whole perceptual apparatus. ${ }^{122}$

Murray understands immersion to be something possible from engagement with most media, including literature and films. However, she is particularly interested in the ways that interactivity and immersion intersect in games. Where a novel can provide the sensation she describes above, immersion in interactive or participatory games require us to "learn how to swim", or to act within the digital environment. ${ }^{123}$ Rather than simply being exposed to immersive experiences, the player participates in creating them. In other words, the player not only suspends disbelief, but also "creates belief" in an active and participatory way. ${ }^{124}$

Murray's understanding of immersion compliment's Cohen's definition of identification. He explains that identification is the mechanism through which audiences experience texts with an insider's perspective, and as if the events were happening to them. ${ }^{125}$ In the process of identification a person "surrenders consciousness of her own identity" and adopts the point-of-view of the character she is identifying with, which leads to a temporary process of "psychological merging" and state of seeing the world through an "alternative social reality" ${ }^{126}$ Audience members may identify with negative

\footnotetext{
122 Murray, Hamlet on the Holodeck, 98.

123 Murray, 99.

124 Murray, 110, 125.

125 Cohen, "Defining Identification," 245.

${ }^{126}$ Cohen, 248, 252.
} 
characters, although Cohen suggests that this is likely to cause "dissonance, guilt, or even fear". 127

Importantly, Murray sees computers (and video games) as liminal objects, "located on the threshold between external realities and our own minds". ${ }^{128}$ Drawing on Winnicott, Murray argues that the power of games, like the power of stories, is derived from the fact that the "real thing is the thing that isn't there". ${ }^{129}$ In other words, games are affectively powerful and immersive because they mask the real with the unreal. ${ }^{130}$ The pleasure is derived from the mix of the real and the unreal. This is something I will return to in my discussion of dark play and its ability to evoke a pleasurable experience of liminality in the following chapters.

However, the appeal of games' liminal space where we experience both the real and the unreal and are both ourselves and outside of ourselves is also anxiety producing. Murray raises several important questions regarding the importance of liminality to our experience of immersion and identification: how can we know that our "imaginary actions" will not have impacts in the real world? And, how can we act on fantasies without being "paralyzed by anxiety" ?131 These questions are taken up by feminist advocates and by the judiciary in a way that grows out of a fixation on the importance of immersion and the risk of slipping between virtual and real worlds.

\footnotetext{
127 Cohen, 252.

128 Murray, Hamlet on the Holodeck, 99.

129 Murray, 100.

130 Murray, 100.

131 Murray, 103.
} 


\section{The Gaze, Voyeurism, and Spectatorial Pleasure}

By virtue of the debates about violence in digital media, one central aspect of this thesis will be unpacking how different audiences understand and interpret GTA 5 differently. I will therefore draw on a multitude of authors who are exploring the heuristics of digital media analysis. One central figure whose ideas have remained important in the feminist analysis of media, is Laura Mulvey. By mobilizing the concept of the male gaze in film studies, Mulvey demonstrated the ways film is informed or shaped by pre-existing social factors, namely sexism and the objectification of women. The mainstream film, she argues, is "hermetically sealed" from the audience, and this sense of separation is what produces the "voyeuristic fantasy". This scopophilic effect, the sexual pleasure derived from looking at another as a source of sexual excitement, reproduces the roles of active/male and passive/female through a form of "sadistic" visual pleasure. ${ }^{132}$

Writing in response, Studlar aims to decentralize the role of the sadistic visual pleasure or the male gaze, as the only source of spectatorial pleasure, and instead emphasizes the masochistic nature of visual pleasure. ${ }^{133}$ Studlar draws on Deleuze in an attempt to demonstrate the shortcomings of the strict Freudian sadism/masochism divide on which Mulvey's argument rests, in the hopes of providing an escape from the interpretive "dead-end" it creates. ${ }^{134}$ Whereas visual sadism is characterized by obscenity, and impersonality, the masochistic aesthetic is characterized by "fantasy,

\footnotetext{
132 Mulvey, "Visual Pleasure and Narrative Cinema," 3, 4.

133 Studlar, "Masochism and the Perverse Pleasures of the Cinema," 267, 269.

134 Studlar, 269.
} 
disavowal (of power), fetishism, and suspense". ${ }^{135}$ This alternative approach allows for a wider range of potential pleasures, and a broader scope of potential psychological influences. ${ }^{136}$ Based on this, she argues that part of the pleasure of film might be connected to the viewer's ability to "assume the position of the child who desires to be controlled within the dynamics of the fantasy". ${ }^{137}$ This fundamentally challenges the idea that male visual pleasure centres only around dominance and control, and can never be rooted in "identification with or submission to the female". ${ }^{138}$

A particularly compelling application of these principles is found in Carol Clover's work on horror movies. Clover's ability to re-read a genre against conventional understandings, and to question theoretical and methodological assumptions that underpin them is an instructive approach for feminist games studies that wants to avoid the pitfalls that Studlar identifies. Clover argues that male viewers are more likely than we might imagine to identify with the pain and humiliation of the (often female) victims in horror movies. She writes that "[slasher films] align their viewers with a female character and give them dose after dose of so-called feminine masochism."139 As Clover suggests, games may well be a way of identifying with masochistic pleasures and hidden fantasies rather than the powerful saviour figure. While this flip in expected identification doesn't necessarily upset hegemonic power relations, it does add an important layer of

\footnotetext{
135 Studlar, 268.

136 Studlar, 269.

137 Studlar, 270.

138 Studlar, 270.

${ }^{139}$ Clover, Men, Women, and Chain Saws.
} 
complexity. Feminist game scholars must be more attentive to her key questions: "what [violence], what [players] and what sort of 'identification' exactly" ?140

This reworking which includes pleasure based on submission has had interesting implications in the analysis of violent media. Popular culture has assumed that the appeal of violent video games is about masculine power fantasies. ${ }^{141}$ While this may certainly be a central experience and appeal for some players, Studlar and Clover tell us that we have to leave other interpretive potentials open. Putting these ideas in play with scholars examining games through a BDSM lens, it seems clear that video games present a particularly rich potential for the sort of loss of control that Studlar addresses.

Following these scholars my analysis here focusses on exploring the unspoken pleasures that exist in the recesses of the debate about GTA 5: those pleasures that are often either unacknowledged or deliberately ignored because they are uncomfortable or problematic. ${ }^{142}$ Of course, this is not an attempt to exculpate problematic media or depictions. I will draw on Studler, Clover, Nash and other scholars to unpack the "dangerous pleasures" of GTA 5 throughout the following chapters. In Chapter 4, I draw on Crane-Seeber's work on "militarized males' masochistic surrender to state authority" to discuss the pleasure that comes with playing GTA 5's infamous torture scene. In Chapter 5, I will turn more directly to Nash, and draw on games scholars such as David Leonard to examine both the injurious aspect of GTA 5's racial depictions, as well as the ecstasy of racial performance.

\footnotetext{
140 Clover, 5.

${ }^{141}$ Sebag-Montefiore, "'Fed up with Fantasies for Male Teenagers'”; Walter, "Let's Be Honest"; Jantzen and Jensen, "Powerplay - Power, Violence and Gender in Video Games."

142 Nash, The Black Body in Ecstasy, 2.
} 


\section{Chapter 3: Methods}

\section{Scope of research}

Because this project will seek to understand how individuals experience violent moments of gameplay, focusing on individual perception of an experience, rather than its external or objective meaning, will be my priority. My hope is that I will add to the growing body of work focused on the importance of gameplay, everyday practice, and player experience in games studies by emphasizing the personal knowledge, perspectives, interpretations and subjectivities of gamers, rather than an objective authoritative interpretation of a game. ${ }^{143} \mathrm{My}$ intent is to push back against the overemphasis on media-effects research, and instead reinforces the importance of play and player. ${ }^{144}$ I believe that this will help us to better understand the complexities and contingencies of the experience of virtual transgression and attend to the "restless negotiation[s]" between player and game. ${ }^{145}$

While much of the continued financial success of GTA 5 has been credited to GTA Online, my research will not address online play, as I am primarily concerned with understanding the experience of solo-play and violence in contexts where the player is engaging introspectively with virtual violence. In online play, there are added ethical considerations because the violence the player experiences may in fact be visited on a proxy of a real-world human.

\footnotetext{
143 Shaw, "Rethinking Game Studies," 349; Taylor, Play between Worlds.

144 Finn, "Political Interface: The Banning of GTA 3 in Australia," 13.

145 Finn, 13.
} 
The methods underpinning this work include phenomenology, autoethnography and textual analysis. Through an in-depth study of player experience and affective response combined with textual readings that challenge taken-for-granted meanings, I aim to show that the meaning of the text is contingent. This methodological and theoretical combination acknowledges the dialogic nature of video games, and rejects an analysis based on an authorial interpretation of a text. Personal memory and history are central aspects of this process of discovery, and the autobiographical nature of both phenomenology and autoethnography necessitate that the researcher is visible, if not centered, in the research ${ }^{146}$.

\section{Phenomenology}

In phenomenological epistemology, rather than seeing a phenomenon as having an inherent and objective meaning, perception and "the experience itself", rather than what might underlie or cause it, is privileged as a key source of knowledge, and as an inexhaustible source of possible meanings, ways of knowing, and experiences. ${ }^{147}$ This requires a "return to the self", and attention to internal workings rather than external interpretations in order to understand and discover phenomenon and experiences. This provides an anti-essentialist interpretive lens when engaging with the text, and challenges positivist assumptions because it does not agree that "natural phenomenon" are objective. Only "truth" comes from what we know of ourselves.

\footnotetext{
146 Moustakas, Phenomenological Research Methods, 59.

147 Moustakas, Phenomenological Research Methods.
} 
While traditional phenomenology has a unilateral concern with the experience, feminist phenomenology recognizes that the experience is itself a site and object of gender ideology and power. ${ }^{148}$ In an effort to recognize this, I will adapt the phenomenological methods of this project to include autoethnography - a method and theoretical approach to which feminists have contributed significantly and that aims to situate experiences within their cultural context. In all cases the self remains central because, as Strivers argues, in feminist research "there is no such thing as removing the observer from the knowledge acquisition process, since to do so would be like trying to see without eyes" ${ }^{149}$

Phenomenology is a particularly apt way of approaching a game like GTA 5 because of the necessity of engaging with Epoche. Epoche is an aspect of the phenomenological method in which the researcher attempts to set aside judgement and the "everyday, ordinary way of perceiving things" ${ }^{150}$ Because the experience is primary, Epoche pushes us towards seeing and "perceiving freshly". ${ }^{151}$ GTA is a game series heavily weighted with preconceived meanings- for example, when I told others I was studying GTA they often asked: "the game where you kill sex workers?". Of course, it is possible to kill sex workers in GTA 5, and the game certainly contains content that can be identified as misogyny (although not necessarily in the forms I had expected). This is why

\footnotetext{
148 Young, Throwing like a Girl and Other Essays in Feminist Philosophy and Social Theory.

149 Strivers, "Stivers, Camilla. 'Reflections on the Role of Personal Narrative in Social Science.' (1993): 408-425.," 410.

150 Moustakas, Phenomenological Research Methods, 33.

151 Moustakas, 33.34.
} 
it feels particularly important to come to the game with the intent of seeing it freshly, to come to it as a "perpetual beginner" as is required in phenomenological analysis. ${ }^{152}$

The challenge of the Epoche, because of the centrality of the self, is to be "transparent with ourselves", and to be "inclined towards receptiveness". ${ }^{153}$ Practically, undertaking the Epoche requires the researcher to dwell with the phenomenon in a process of "unusual, sustained attention, concentration and presence". ${ }^{154}$ Most resources about phenomenological methods don't provide concrete advice on performing this, and I felt that a more guided form of intention is needed during this stage of my research. I found the process of autoethnography to be a helpful way of thinking about Epoche, and relied heavily on this methodology in the early stages of my research.

\section{Autoethnography}

The first phase of my project consisted mainly of autoethnography. As such, it has also precluded any chance that this research be seen as objective or generalizable and has instead foregrounded subjective experience. In autoethnography, as in phenomenology, the author's subjectivity and bodily standpoint is a source of knowledge that rejects objectivity or authorial omnipresence. ${ }^{155}$ The most instructive definition of autoethnography is provided by Carolyn Ellis:

Autoethnography refers to writing about the personal and its relationships to culture. It is an autobiographical genre of writing and research that displays

\footnotetext{
152 Sallis, "The Identities of the Things Themselves" quoted in Moustakas, Phenomenological Research Methods, 86.

153 Moustakas, Phenomenological Research Methods, 86.

154 Moustakas, 87.

155 Denzin, "The Many Faces of Emotionality: Reading Persona," 20 quoted in Spry, "Performing Autoethnography," 711.
} 
multiple layers of consciousness [...] Back and forth autoethnographers gaze: First they look through an ethnographic wide-angle lens, focusing outward on social and cultural aspects of their personal experience; then, they look inward, exposing a vulnerable self that is moved by and may move through, refract, and resist cultural interpretations. As they zoom backward and forward, inward and outward, distinctions between the personal and the cultural become blurred, sometimes beyond distinct recognition. ${ }^{156}$

Autoethnography is an active process, characterized by movement and tension.

While personal narrative is certainly an important aspect of the process,

autoethnography is also an experiment that requires the author to question, unpack, examine and reflect on themselves as a subject. While autoethnography helps to provide thick ethnographic descriptions of experiences, it also requires that we interrogate our own assumptions in a way that helps the researcher become simultaneously an insider and an outsider to their own experiences. ${ }^{157}$

Using autoethnography also provides me with a way to reflect on the challenges of studying games as a woman. While I experience privilege in a number of ways in the broader world, as a woman I remain on the margins of the video game world. Autoethnographic approaches are particularly resonant with women or other marginalized players because they emphasize the woman's role as a valid player, thereby helping to contradict the reification of the "presumed male player". In doing so, autoethnography draws attention to the (relatively, in my case) marginal position of the author while simultaneously acknowledging and validating their presence. ${ }^{158}$ This is particularly important because of the challenge of doing interviews with male players

\footnotetext{
156 Ellis, The Ethnographic I, 38.

157 Richards, "Writing the Othered Self: Autoethnography and the Problem of Objectification in Writing About IIIness and Disability," 1721.

158 Chávez, “Autoethnography, a Chicana's Methodological Research Tool," April 2012, 1547.
} 
about transgression. Both of my male participants acknowledged that gender tends to change the way play happens:

AARON: I think a lot of men feel less comfortable being... letting loose [in their gameplay] in front of women. I've never felt like that, I went to a high school that was two-thirds women, I have a little sister, I've always had more female friends than men, so it's not an issue for me. But it changes that male dynamic, the locker room talk element of [the social experience of gameplay].

CONNOR: Yeah, whenever there's a girl [playing online] it's always like, a thing, whether you comment on it or not, someone always says something. Yeah, it's weird. Not weird... like it doesn't make you feel weird, but the whole situation is like "oh there's a girl here", and it really matters, but no one knows why.

Adding to this difficulty, some of the most important themes in my own play were masochism, psychosexual desire and rape fantasy, among others. Attempting to understand whether male players shared these experiences was a challenge. Not only in terms of developing rapport over a relatively short interview, but because the presence of a woman tends to disrupt the nature of transgressive gameplay in and of itself.

\section{Textual Analysis}

I also engage in hermeneutical textual analysis in combination with my autoethnography. This form of analysis aims to explore possible understandings rather than provide an authoritative reading of the text and emphasizes the situatedness of each interpretation while paying attention to the presuppositions that the researcher brings to the analysis, in much the same way phenomenology and autoethnography do. ${ }^{159}$ The interpreter is understood as having an active role, as opposed to analytical approaches that attempts to take an omnipresent or authoritative view. Further,

\footnotetext{
159 Kinsella, "Hermeneutics and Critical Hermeneutics: Exploring Possibilities within the Art of Interpretation."
} 
hermeneutics is comfortable with ambiguity, which is particularly important to my analysis. $^{160}$

Rather than attempting to objectively analyze GTA 5 or organize the meaning of the text into "tidy categories", the focus of this sort of interpretivist textual analysis is on the interpretative process that leads to the subjective understandings of a text. ${ }^{161} \mathrm{My}$ interviews supported this by helping me to understand how my participants constructed their interpretations of GTA 5. Throughout the text I also draw on online forums where players talk about the game. My analysis of these forums is certainly not quantitative, nor is it necessarily representative. There are, of course, many drawbacks of relying on anonymous posts. However, I feel that these small injections from players often help to illuminate my own interpretations and are thus hermeneutically valid.

\section{Procedures:}

My research unfolded in three phases. First, I conducted an autoethnographic exploration of GTA 5. Second, I conducted in-depth interviews with a small number of participants. Finally, I analyzed the data collected during the autoethnographic and interview phases. Below, I have provided a detailed description of this process.

\section{A. Phase One: Autoethnographic Let's Plays}

Let's Plays are a type of video that documents video game play, with a screen capture of the player nested in the main window which shows the game as the player

\footnotetext{
160 Kinsella.

161 Lacity and Janson, "Understanding Qualitative Data: A Framework of Text Analysis Methods."
} 
would see it (Fig.1). The player often provides continuous commentary as they play.

While Let's Plays are a tool most often used for live-streaming by video game websites or online personalities, Sapach has convincingly demonstrated their usefulness for researchers trying to conduct autoethnography of video games where they prove to have a marked advantage over more traditional ethnographic notes or journals.

Although similar approaches exist in visual ethnography, Let's Plays give a researcher more access to their own experience than they might otherwise have, because they allowed me to feel that I could immerse myself in the game as I usually would, while also being able to have access to a record of this experience which included information (such as facial reactions) that I would not otherwise have had. ${ }^{162}$ The ability to re-watch your own play, and to evaluate and analyze expressions, reactions and movements is invaluable. In many ways, it opens up opportunities for further experience: I experience the violence in the game, but I can also re-experience and re-perceive the outward appearance and impact of that violence on my body (both digital and flesh). ${ }^{163}$ This method also helps to reorient analysis around the players' experience, rather than a purely textual analysis. Further, it supports the phenomenological imperative to examine an experience from many angles, and to distill it down to its essence which requires at least a full recollection of the experience, and ideally the opportunity to re-experience the phenomenon.

\footnotetext{
162 Sapach, "Let's Play with Research Methodologies: Autoethnographic Memory Work and Critical Theory Development Through a 'Let's Play' of TRAUMA"; Pink, Doing Visual Ethnography.

163 Moustakas, Phenomenological Research Methods, 55.
} 
The fact that I am on-camera also introduces complexities that might not be as apparent in written field notes: the player may alter their play or present themselves differently because they know they are being recorded. This may be unconscious, or there may be a deliberate performance for the camera or, on the other hand, a form of self-monitoring or self-censoring. ${ }^{164}$

\section{B. Phase Two: Interviews}

I take after Keogh in borrowing from Interpretative Phenomenological Analysis (IPA) to structure my interviews. ${ }^{165}$ IPA is concerned with understanding an experience on its own terms, and why and how experiences take on significance in people's lives. ${ }^{166}$ After being granted ethics clearance by the Carleton University Research Ethics Board, I began recruiting participants for interviews. Participants were recruited mainly through the snowball method. I initially shared the information about my project with key friends and acquaintances who I knew would know people who had played the game. Potential participants were asked to contact me, and I would then ask them to complete a short series of screening questions to determine their eligibility. I was then able to select participants from a pool of potential interviewees.

Before the interview, selected participants were sent a letter of invitation, and signed an informed consent form. Interviews were scheduled to take between 60-90 minutes and consisted of open-ended conversation about the player's impression of the

\footnotetext{
164 Sapach, "Let's Play with Research Methodologies: Autoethnographic Memory Work and Critical Theory Development Through a 'Let's Play' of TRAUMA."

165 Keogh, "The Positive Discomfort of Spec Ops: The Line."

166 Smith, Flowers, and Larkin, Interpretative Phenomenological Analysis, 1.
} 
game, experiences in the gameworld, and the relationship between these experiences and the 'real world'. In total, seven individuals expressed interest. All were men between 18 and 27, and all but one were white. From this pool, I selected two white men between 26-27 to interview. ${ }^{167}$ IPA calls for small samples of between three and six to allow for indepth engagement, with a focus on relatively homogenous participant pool to facilitate exploring the similarities and differences between each participant. ${ }^{168}$ In this case, I treated my own autoethnography as the first case, and chose my participants because they were in the same age range and the same race as me. Given the themes of masculinity immerged so centrally from my own play, I also felt it was important to explore this axis of difference through the interviews. All participants reported playing over 100 hours of GTA 5. Interviews were done in-person and each was approximately an hour and a half long, I also conducted a half-hour follow up interview with one participant. Interviews were audio recorded and transcribed.

\section{Phase Three: Analysis}

I am basing my method for analyzing the transcripts on Clark Moustakas' modification of the Stevick-Colaizzi-Keen method of analysis of phenomenological data, which he outlines in his book, Phenomenological Research Methods. The steps taken are outlined below:

\footnotetext{
167 While I conducted three interviews, one of the participants was dropped during the analysis phase. This was because the interview occurred over the phone, and I struggled to establish familiarity and rapport. The interview was therefore more superficial than the others.

168 Smith, Flowers, and Larkin, Interpretative Phenomenological Analysis, 48-49.
} 
1. For each participant's transcript, the following steps are taken:

a) Each statement is evaluated with respect to its significance in terms of describing and explaining the experience.

b) All relevant statements are recorded, repetitive and/or overlapping statements are excluded.

c) The relevant statements make up the horizons or meaning-units of the experience. This process of horizontalizing requires that each statement relevant to the topic or question is seen as having equal value (p.118)

d) Horizons are then grouped or clustered into related themes.

e) Horizons, themes and verbatim examples are then synthesized into a description of the texture and structure of the experience. Textural description aims to capture the feelings, emotions and embodiment of the experience for the participant. Structural descriptions, on the other hand, aim to account for the "underlying dynamics of the experience", and the themes and qualities that account for "how" feelings and thoughts are aroused and what conditions bring the experience on.

2. Based on the textual-structural description of all participant's experiences, individual descriptions are brought together to construct a composite "textualstructural description of the meanings and essences of the experience".

3. These descriptions are interwoven throughout a broader analysis that includes my autoethnography. 


\section{Limitations}

As Philaretou and Allen explain, while autoethnography holds considerable potential for researching contentious or delicate subject matters, there are also challenges associated with it. These challenges are often personal, as they explain: "In trying to systemically articulate the psychological, emotional, and sexual variants of one's personal life, an autoethnographer comes face to face with the negative aspects of his or her self-concept that may be hard to acknowledge, understand and accept". ${ }^{169}$ Therefore, autoethnography challenges the stability of the self, as it demands that the researcher externalize and analyze what is normally private, which "unmasks its limitations and weaknesses". ${ }^{170}$ The research is thus limited by both the ability of the researcher to access their own experiences, feelings, and to their success in stepping back from these and applying theoretical analysis to it, but also by the potential that they may self-censor or, in more extreme cases, conceal, information. ${ }^{171}$ As Chávez explains, the fact that we "must rely on our fragmented sense of memory or our limited capacity to understand and reflect upon our own experiences" is an inherent limitation of autoethnography. ${ }^{172}$ Furthermore, as a method that often counter-positioned itself with the academic establishment, autoethnography is occasionally "viewed with suspicion in the academic world, [and seen as] universally self-indulgent". ${ }^{173}$ Resisting this, Sparkes

\footnotetext{
169 Philaretou and Allen, "Researching Sensitive Topics through Autoethnographic Means," 5.

170 Philaretou and Allen, "Researching Sensitive Topics through Autoethnographic Means," 5.

171 Philaretou and Allen, 5.

172 Chávez, "Autoethnography, a Chicana's Methodological Research Tool," April 2012, 345.

173 Smith and Sparkes, "Narrative and Its Potential Contribution to Disability Studies," 25.
} 
argues that "autoethnographies can encourage acts of witnessing, empathy and connection that extend beyond the self of the author" ${ }^{174}$ Therefore, while autoethnography may be more vulnerable to critique than other methods, it is particularly well suited to my project.

Autoethnography is a particularly valuable method when dealing with sensitive topics or experiences such as those discussed throughout this project. However, this comes at the expense of the research being generalizable. ${ }^{175}$ In order to help counterbalance this limitation, I have attempted to triangulate my data through the use of several qualitative methods. I believe that the contributions of each make the findings more robust, albeit still subjective. ${ }^{176}$ That said, generalizability here is not the intent. Instead, I see this "limitation" as an inherent value. Autoethnographic methods help to "delineate the complexity, totality, and unpredictability" of human experience, and can therefore help to destabilize the taken-for-granted assumption often made about our experiences with violent games, thereby contributing to future explorations and research. ${ }^{177}$

Interpretative Phenomenological Analysis (IPA) has its own set of limitations. It has a purposefully small and homogenous sample group, which means that, like autoethnography, the method is better suited to exploring the range of possible experiences and interpretations, rather than delineating a generalizable conclusion about the experience itself. IPA focuses on having participants discuss and describe their

\footnotetext{
174 Smith and Sparkes, 25.

175 Chávez, "Autoethnography, a Chicana's Methodological Research Tool," April 2012, 345.

176 Philaretou and Allen, "Researching Sensitive Topics through Autoethnographic Means," 6.

177 Philaretou and Allen, 7.
} 
experiences in a way that "stresses self-expression, emotion, and subjective accounts" ${ }^{178}$ While this is an important counterbalance to those textual game studies that ignore or minimize the role of player interpretation, as Keogh points out, IPA and phenomenological methods that involve study participants more broadly mean that the researcher is always twice removed from the experience itself. ${ }^{179}$ It can be thought of as a "double hermeneutic experience" as the researcher tries to "make sense of the participant trying to make sense of what is happening to them". ${ }^{180}$

This can cause challenges because discussions may reveal more about player's attitudes and interpretations rather than their experiences. ${ }^{181}$ I found this to be true in my own interviews, where I often felt I was learning more about a player's attitude towards games generally, or how they come to make sense of public discourse, rather than how they experienced specific moments, and the subjective emotions this raised. This was certainly compounded by another challenge that Keogh identifies: significant time had passed since players last played the game. ${ }^{182}$ As with his study participants, the discussions with my interviewees show that the experience of playing the game was memorable to them, although the experiences they remember most vividly may not be representative of their entire play experience, and their responses likely address their "retrospective interpretations" of the experience, rather than the experience itself. ${ }^{183} \mathrm{My}$ way of addressing this has been to use the interviews in a way that supports the

\footnotetext{
178 Keogh, "Videogames Aren't Special. Videogames Aren't Unique.," 4.

179 Keogh, "The Positive Discomfort of Spec Ops: The Line," 4.

180 Smith, Flowers, and Larkin, Interpretative Phenomenological Analysis, 3.

181 Keogh, "The Positive Discomfort of Spec Ops: The Line," 4.

182 Keogh, "The Positive Discomfort of Spec Ops: The Line."

183 Keogh, 4.
} 
autoethnographic pillar of this research. I view the interviews as moment of phenomenological intersubjectivity. By sharing experiences, Husserl believed that there was an "interchange of perceptions, feelings, ideas and judgements", and potential validation, about the nature of the experience and reality. ${ }^{184}$ The interviews therefore provided an opportunity for validation or correction of my own experiences and were helpful in providing a more accurate description of the nature of the play experience.

${ }^{184}$ Cerbone, Understanding Phenomenology. 


\section{Chapter 4: Transgression and dark play}

The human spirit is prey to the most astounding impulses. Man goes constantly in fear of himself. His erotic urges terrify him. The saint turns from the voluptuary in alarm; she does not know that his unacknowledged passions and her own are really one.

- Georges Bataille, Foreword to Eroticism

The theme of transgression emerged early on in my own playthrough of GTA 5 . The first couple days of play were unremarkable in terms of violence, which I commented on in my gaming journal. However, by the second and third day of play it became clear that while GTA 5 was not a violent game in the way some others I had played were (where it's commonplace to hack, slash and shoot your way through the world, dismembering countless opponents along the way), there was something about GTA 5 that gnawed at me in a way that other games didn't always; it often felt more violent than it actually was, and in my gaming notes I wondered if thus was because it tended to pull at more deepseated anxieties than other games.

In this chapter I aim to explore this connection between discomfort, anxiety and pleasure in order to frame the discussion that follows in the final chapters. I draw a parallel between the concepts of transgression and dark play and argue that dark play is essentially playful transgression within the context of a game. The value in making the equation between transgression and dark play is that it highlights the ways in which dark play is linked to eroticism. While research on dark play has certainly focused on sexual play that might be considered controversial, linking the two concepts helps to show that 
most dark play, whether or not it includes explicitly sexual elements, is erotic in the Bataillian sense because it encourages a dissolution of self. These erotic elements of dark play are best understood through a kink-informed analytical lens.

Being a game, there are particularities we have to attend to, namely that while players are able to experiment with transgressive behaviours (such as stealing cars), these acts are condoned within the rules and mechanics of the game. Therefore, while the GTA series seems to derive at least some of its appeal from the fact that it allows players to experience the pleasure and thrill of transgression, it does so in a relatively safe way. While the experience of transgression and dark play is characterized by liminality and a dissolution of self, in the context of a game players can minimize their feelings of anxiety or discomfort and foreground the experiences of pleasure, thrill and exploration that transgression affords them. At the same time, as my autoethnography and interviews show, it's the holding of the real and the virtual in tension with one another that allows dark play to happen and makes it enjoyable. This pushes back against essentialist understandings about the relationship between video games and real-world violence by complicating the relationship between the real and the virtual and by advancing a more nuanced understanding of what draws players to transgressive play.

\section{Transgression}

The player's ability to "do anything", in the Grand Theft Auto series is often touted as one of its biggest draws, as well as one of its most dangerous characteristics. While anything can include competing in triathlons and playing tennis, it's the ability to engage in transgressive play with violence, immorality and taboo that seems to captivate 
the imaginations of both players and detractors. Transgression can be simply defined as norm-breaking, of either major or minor standards. ${ }^{185}$ However, it is often a much more slippery concept than that. Throughout this thesis, I use transgression to refer to play that subverts norms in some way. Talking about transgression necessitates an examination of the norms and taboos that help to delineate the boundaries of social acceptability. Because norms and taboos influence us all, the appeal of transgression is much broader than conventional radical feminist approaches would have us believe. As one participant put it, transgression is a manifestation of "something inside of us" that wants to "do something you wouldn't in everyday life".

Stallybrass and White draw on Babcock's concept of symbolic inversion in their definition of transgression, quoting:

Symbolic inversion may be broadly defined as any act of expressive behaviour which inverts, contradicts, abrogates, or in some fashion presents an alternative to commonly held cultural codes, values and norms [...] ${ }^{186}$

Rather than being either hegemonic or transformative, transgression serves a dual role: to both circumscribe a culture's boundaries and, in doing so, draw into question their apparent objectivity and inevitability. ${ }^{187}$ By its definition, transgression points to and makes visible the codes, values and norms which give it its affective power and meaning. By the same token, those cultural standards make transgression "a structural inevitability". ${ }^{188}$

\footnotetext{
185 Newall, "An Aesthetics of Transgressive Pornography."

186 Babcock and American Anthropological Association, The Reversible World, 14 quoted in Stallybrass and White, The Politics and Poetics of Transgression, 17.

187 Babcock and American Anthropological Association, The Reversible World, 29.

188 Stallybrass and White, The Politics and Poetics of Transgression, 46.
} 
Unlike Bakhtin's formulation of the carnivalesque, transgression is not necessarily sanctioned, nor does it either support or disrupt hegemonic power or social systems. While I argue that the GTA series allows players to engage in inversion and transgression, I do not argue that these are necessarily positive or negative. While players may certainly experience the "the exhilarating sense of freedom which transgression affords", this affective experience does not necessarily indicate transformative or progressive potential. ${ }^{189}$ This is an important distinction from the polarized approaches to pornography that characterized the Sex Wars.

For my purposes, one of the most important aspects of transgression is its connection to eroticism. Bataille believed that eroticism depends fundamentally on the existence of taboos, and the pleasure of breaking them. Without taboo, there can be no eroticism. ${ }^{190}$ He argues that eroticism always involves disassembling "established patterns" that regulate the social order and enforce a condition of discontinuity, isolation or separateness. ${ }^{191}$ Bataille saw transgression and eroticism as a way of inverting this condition, at least temporarily, and of moving towards continuity or the breakdown of the self as an isolated unit.

This move from discontinuity (or separateness, isolation) to continuity is the central character of eroticism, which Bataille argues is aimed at "destroy[ing] the selfcontained character of the participators as they are in their normal lives" ${ }^{192}$ The opening up of the body to continuity provokes uneasy or perhaps uncanny feelings which he

\footnotetext{
189 Stallybrass and White, 27.

190 McCabe, "Introduction to the Penguin Edition," $x$.

191 McCabe, 18.

192 McCabe, 17.
} 
argues we consider to be obscene because they are counter to our sense of selfpossession. This is connected to Freud's understanding of transgression, in which transgression is seen as a particularly rich source of both anguish and ecstasy, and thus of psychic disturbance. ${ }^{193}$

This movement from discontinuity to continuity is hypnotizing, intoxicating and erotic. While scholars, including Delamere and Shaw, argue that the pleasure of violence in video games is about gaining control and power, Bataille's conceptualization of the transgressive involves a loss of power through loss of self. ${ }^{194}$ Rather than gaining mastery, transgression is appealing because of the way it allows us to step to the precipice of "death", or a loss of control. Therefore, eroticism as the product of transgression, is characterized by a fundamental division of self, or a "rending orgasm" in which the subject experiences a "partial dissolution" of their normal discontinuous self. ${ }^{195}$

This is important to my argument because radical feminist understanding of violence often links it to sexuality. ${ }^{196}$ I agree that there is a connection between the violent themes found in video games and eroticism, however, unlike radical feminist conceptualizations, I don't believe that we can draw easy conclusions about the nature of power and control based on this alone. Instead, a kink-centric lens that explores the relationship between violence, transgression and pleasure, and supported by autoethnographic exploration and the experiences of my interviewees, allows us to take a nuanced approach to the analysis of transgressive gameplay.

\footnotetext{
193 Hester, Beyond Explicit, 85.

194 McCabe, "Introduction to the Penguin Edition," xiii.

195 McCabe, 17.

196 MacKinnon, "Sexuality, Pornography, and Method: 'Pleasure under Patriarchy'”; Dworkin, Pornography.
} 
While the authors discussed here provide a theoretical framework for thinking about the nature of transgression and its relationship to cultural norms and taboos, turning to leisure studies helps to apply this theory to the everyday practice of gameplay focused on controversial or taboo play. The concept of dark play is particularly useful for understanding how game players engage in transgressive behaviour.

\section{Dark Play}

The concept of Dark Play has been taken up by leisure and games studies as one way of understanding play that includes controversial themes and play behaviours (such as deception or "griefing"). The concept has its roots in performance studies, and was introduced in Schechner's 1988 work, Playing. ${ }^{197}$ In their anthology about dark play, Linderroth and Mortensen adapt Schechner's definition and expand the concept to encompass any "playful transformation" of dark themes in games. "Dark" refers to game elements that might be considered "problematic, subversive, controversial, deviant or tasteless" ${ }^{198}$ As with symbolic inversion, the authors point to the fact that these themes undergo a transformation or subversion through play: "dark play subverts order, dissolves frames, and breaks its own rules- so much so that the playing itself is in danger of being destroyed" ${ }^{199}$ While its central characteristic is subversion, dark play, like transgression, is not inherently transformative or progressive but rather takes its meaning in relation to cultural norms and standards. In other words, as Brown writes,

\footnotetext{
197 Schechner, "Playing." Schechener's definition of dark play concerns play where some player are unaware that they are partaking in activities that others consider to be playful. In this formulation, dark play is the "subversion of [the] meta-cues that tell us actions and utterances are supposed to be understood as playful", making it deceptive. 198 Linderoth and Mortensen, "Dark Play: The Aesthetics of Controversial Playfulness," 5.

199 Linderoth and Mortensen, 5.
} 
without the "primary moral frame", or the system of normative ethics possessed by players in the real world that outlines acceptable behaviour, "dark play would not necessarily be defined as dark". ${ }^{200}$ Games are particularly interesting in terms of dark play, because much of the behaviour allowed within the game has been condoned by the developer and may be considered acceptable within the game world. However, as I will discuss at length below, when we approach dark play with a kink-informed lens it's possible to see that while players need to feel safe in their transgression, they also want to feel the push and pull of their real-world ethics. For example, BDSM scenes involving race play are a "game" in that they have a secondary ethical frame and set of rules that guide them. However, as my autoethnography showed me, they are often rooted in real racism and stereotype, and this authentic transgression is what gives the scene its erotic power. I will return to this example in depth in Chapter 6.

Linderoth and Mortensen recognize that playfulness is a "state of mind" that is filled with tension and hinges on a "precarious balance" that needs to be both maintained but simultaneously put at risk for play to be interesting. They therefore understand playfulness or the play state as being created by the intersection of several points of tension, meaning that the player is "suspended" in a playful space rather than in an isolated or clearly demarcated "protected space", and able to keep several thoughts in play at the same time. ${ }^{201}$ The player's capacity to maintain playfulness when engaged with controversial issues including "violence, sadism, and racism" depends on their ability

\footnotetext{
200 Brown, "Three Defences for the Fourteen-Inch Barbed Penis: Darkly Playing with Morals, Ethics, and Sexual Violence," 123.

201 Linderoth and Mortensen, "Dark Play: The Aesthetics of Controversial Playfulness," 6,8.
} 
to balance between their real world selves and ethics and the way the game applies pressure to these, which destabilizes their self and sense of morality. This mirrors Bataille's conceptualization of the erotic as the dissolution of self. ${ }^{202}$ In dark play, the player experiences a shift from self-possession to a more liminal space where "what is and what might be" exist simultaneously. As I will discuss in the subsequent chapters, my autoethnography and interviews confirmed the importance of this balancing act.

Dark play is rarely stable. It's meaning shifts and changes as players negotiate the tensions they are faced with. How this unfolds will depend on a wide variety of factors in both the real and virtual worlds. In addition, understanding dark play requires that we understand both the text, and the play experience.

A particularly apt example is found in Schott and Van Vught's study on parents' preconceptions about GTA 4 and how these changed when they played it themselves. The researchers quote one parent, who explains:

[The game] goes against the values I am trying to instill in my children about respecting authority and you don't kill cops and you don't run over prostitutes, you know, there's no respect for life in it, I think, is what I rebel against.... It's a violent game". ${ }^{203}$

While this is a fair textual reading of some of the activities that are possible in the GTA series, it fails to capture the complete affective experience of playing the game. ${ }^{204}$ The act of transgressing taboos or social codes is often experienced as both pleasurable and shameful for the person doing the transgression, whereas simply witnessing the

\footnotetext{
202 Linderoth and Mortensen, 9.

203 Schott and van Vught, "Replacing Preconceived Accounts of Digital Games with Experience of Play: When Parents Went Native in GTA IV," 4.

204 Schott and van Vught, 2.
} 
transgression or talking about the idea of transgression may not have the same impact.

While parents like the one above initially expressed trepidation about the game as a deviant or dangerous piece of media, as they played the game they began to experience dissonance between this anxiety and their pleasure in play:

In one case, a participant was in the process of narrating how objectionable it was that you could run over pedestrians in a game, when he turned a corner in his car, mounted the pavement, and squashed a pedestrian against a wall. At that moment, the participant was unable to contain his laughter, undermining his rational evaluation of the game with his bodily and nonverbal response. ${ }^{205}$

Similar reactions occur countless times throughout the Let's Plays that document my experience. I would hit a pedestrian and with a sickening crunch they would fly up and over the hood of my car. My grimace would morph into a half-smile or laugh as I called out "sorry!" and accelerate my car, leaving them rolling down the road behind me. Most experienced game players are familiar with this dissonance between violent action and reaction and the pleasure that comes with it. While players often find ways of justifying these sorts of actions, taking a dark-play approach allows us to move past that sort of public-facing rationalization and examine the experience and its associated affects themselves.

The dissonance that exists between rational thought and social norms (violence is bad) and the experience of game play (violence is funny, fun, pleasurable) presents a challenge to essentializing readings. Dark play is affectively powerful experience precisely because it cannot always be rectified or justified, despite players' tendency to excuse it in this way. While players may seek to justify their pleasure (for example, by citing the social

${ }^{205}$ Schott and van Vught, 5. 
commentary of the game) they do not seek to overcome discomfort. Rather, the affective power of the game comes from the ability of players to hold their pleasure and anxiety in tension with one another.

Aaron described the exploration of the game as "exhilarating". For him, exploration involves both looking for new and different things (new cars to steal, for example), but also "seeking out the shocking aspects" (including going on a side mission with a friend to find sex workers). Violent play was an important part of this exploration:

AARON: But the violence aspect, I mean yeah you wouldn't play it if there wasn't the violence... it would just be The Sims! Not that there's anything wrong with The Sims, but yeah that's the aspect that draws you to it: Doing things you would never... robbing a bank, or you know, all that stuff that you could never do in real life.

The connection between the fact you can explore things you would never do in real life and the thrill of violence is an important one. The player acknowledges the social norms preventing the behaviour, but also recognizes that he takes pleasure in transgressing these boundaries, albeit in a relatively safe way. Talking about why he enjoyed playing the character Trevor, Aaron compared him to the character Rorschach from the Watchmen. Both are characters that he gravitated towards and found compelling. His explanation of why these characters are appealing is centered on the idea that transgression is enjoyable:

AARON: I think it's because it's different, its exotic in some ways, but also because there's something inside of us that wants to break those norms and not be held back. It's part of the fun of the game, isn't it? It's things you wouldn't do in real life.

Similarly, Delamere and Shaw found that players enjoyed a gaming-specific thrill from "enacting socially anomic behaviours without concern for any real-world 
consequences". ${ }^{206}$ The quote from one of their study participants is strikingly similar to the quote from my participant above:

I think it, for me anyways, I'm sure a lot of gamers would agree, is it's compelling to be able to play something that you know you wouldn't be able to do in real life, and, you know, get away with it or see what happens, you know. It is fun to have like all the cops chasing you, with none of the real-life consequence, [long contemplative pause] Perhaps the curiosity of, you know, no sane person would do a lot of those things, but here in this virtual world, we can do it without consequence. So let's try and see what happens and you know, a lot of people can justify it because you know, it's no harm done. (participant J.J.) 207

Importantly, these players don't express a desire to explore these same transgressions in real life. Indeed, as we see above, players explain that they find these transgressions pleasurable specifically because of the lack of consequences. This is also connected to immersion and realism; the transgressive thrill of the game was linked to the game's ability to "be as realistic as possible without being realistic". Aaron explains:

AARON: You know it's not real, you know they're just numbers on a screen, so it lets you- "hey, wouldn't it be crazy if I drove a car on the sidewalk, wouldn't it be crazy if I stole an airplane" - right? So, there is that element of catharsis and exoticism, stuff you wouldn't do in real life. You just sort of have fun with it in a world where none of it matters".

Aaron is using the term catharsis to describe the affective experience of safe transgressive play. He explains:

AARON: [Catharsis] for me is more about escape and a break from reality [...] it's like taking a vacation from consequences [...] in the short term you're taking a vacation from what things really matter and engaging in almost the ultimate parody of life.

\footnotetext{
206 Delamere and Shaw, "Playing with Violence," 15.

207 Delamere and Shaw, 15.
} 
While behaviour that is considered transgressive in the real world (stealing cars, dealing drugs, murdering pedestrians) is often condoned and even rewarded by the game, it is clear that for at least some players the ethics and morality of the real world still informs their transgressive play. Some ludological approaches to dark play, including exploration of dark sexual play, depend on the argument that dark play is acceptable or justifiable because it can be rationalized under the ethical framework of the in-game world. ${ }^{208}$ However, this analysis erases the importance of the taboo for transgressive play.

While players certainly identify that the game provides them with an escape from reality, that does not mean that reality does not inform their play experience. As Bataille tells us, to eliminate the taboo is to eliminate the transgression itself, as well as any associated pleasure. While the norms in-game may be more permissive, or even encourage "dark" behaviour, players rarely cordon their real-world moral framework, experiences and feelings off from their virtual play. In Brown's formulation, the taboo itself is erased and even made normative through the evocation of the game rules, making transgression impossible. In this case, the player's sense of self remains intact, there is no dissolution of self.

Conversely, my understanding of dark play requires an acknowledgment that players experience a push and pull between various in-game and real-world moral frameworks. ${ }^{209}$ Whereas Brown draws on Sicart to argue that dark play represents a shift

\footnotetext{
208 Brown, "Three Defences for the Fourteen-Inch Barbed Penis: Darkly Playing with Morals, Ethics, and Sexual Violence."

209 Brown, 122.
} 
in ontology, and a creation of a new subjectivity that aims to be faithful to the ontology of the game (its rules and game world)s, I am arguing that no such shift occurs, or at least not perfectly. ${ }^{210}$ Instead, players experience a fracturing of ontology and self. This, in some ways, represents what Bataille might characterize as a move from discontinuity to continuity. The player experiences a temporary destruction of the self as they inhabit a shared "meta-awareness" between themselves and their avatar. This shared space is characterized by both pain, anxiety and pleasure.

This helps to circle back to Mortensen's argument that players engage with the rules, the narrative, and the moral norms of the real-world in their interpretive efforts, rather than simply abandoning them for a new in-game ontological framework. While players may accept the limitations of the game world and technical game-play, the fiction of the game also informs this process, as does their experience of the real-world. Mortensen argues that the fiction "offers a second set of rules" and that the player plays both within the "rules of the game and the rules of the fiction", which in turn are balanced against their real-world identities and values. ${ }^{211}$ It is not simply the creation of an in-game moral framework that results in the pleasure of transgression, but rather the tension between the real world and game world and their respective ethical frameworks that provides players with the duel affects of pleasure and pain in transgression. ${ }^{212}$

In her study of players of Star Wars: The Old Republic, Mortensen argues that while players do display a tremendous amount of reflection about the ethical

\footnotetext{
210 Sicart, The Ethics of Computer Games quoted in Brown, "Three Defences for the Fourteen-Inch Barbed Penis: Darkly Playing with Morals, Ethics, and Sexual Violence," 123.

211 Mortensen, "Keeping the Balance: Morals at the Dark Side," 163.

212 Mortensen, "Keeping the Balance: Morals at the Dark Side."
} 
implications of their actions, their subsequent actions are not only based on guilt, but also on pleasure. The pleasure here is not derived from a desire to actually conduct immoral or unethical actions or from a "substitute reality", but rather from the opportunity the game provides to reflect on ethical complexity. ${ }^{213}$ Players may therefore rely in part on a set of meta-level morals, playing along with or adopting the moral framework of the game rather than that of the real world, although they continue to put those into conversation with their own "real" ethical framework. Mortensen uses the example of an interviewee who, playing the role of a Sith in the Star Wars universe, acted according to that character's morals which, despite the reprehensible actions, results in a pleasurable meta-awareness characterized by: "the pain of moral ambiguity, the pleasure of acting correctly in-game, and the pleasure of being able to play the role he has chosen in the game well, despite his own ambivalence". ${ }^{214}$ However, these do stand alone, but are held in tension with reality, and the primary moral framework. When this balance is disrupted, the results are not pleasurable. For example, Aaron talked about finding yourself engaged in a shoot-out on an in-game campus as one moment that "points to realism" and triggers anxiety and guilt responses. He explains:

AARON: People are running and screaming, and you make connections to those audio clips you've heard of horrible things that have happened and that triggers those responses of "ooo that just got a bit too real" or "I'm not as comfortable with this as a couple minutes ago" ... because it makes that connection, jumped over that boundary.

\footnotetext{
213 Mortensen, 159.

214 Mortensen, 162.
} 
Moments like this may result in feelings of guilt. For Aaron these feelings were provoked by contemplating whether this division between reality and fantasy is more tenuous than he would like to imagine:

AARON: I find this game, more than a lot of other games, produces feelings of guilt. Or like, there are times when you're thinking about [...] 'is the fact I'm doing this, even fake doing this... does that make me a terrible person'

Importantly, Mortensen argues that it is not immersion or mistaking the game for reality that results in the player's pleasure. Instead the game's ability to destabilize or alter reality is what allows players to experience and explore things that would be impossible in the real world in relative safety. They are nonetheless still able to achieve the affective thrill and experience of liminality, or ethical push and pull, that results from their transgressions. ${ }^{215}$

Mortensen argues that players of games with ethical or morally problematic worlds are often unable to accept this world at face value. On the contrary, she argues that an important part of their enjoyment is the fact that dark play, like transgression, make the social norms and convention of the real world visible, "displaying the weaknesses in what we accept without question", and actually reducing the suspension of disbelief. ${ }^{216}$

At this stage, it's worth acknowledging the differences in the experiences of my interview participants related to transgression. While Aaron's experiences were similar to my own, Connor's experience was different. He seemed to feel the affective potential of

\footnotetext{
215 Mortensen, 167.

216 Mortensen, 168.
} 
transgression far less than us. Aaron expressed ambivalence towards the game's violence, explaining that it was a central appeal of the game, but also that he needed to "justify" liking it to himself, and that he understood that it might be stigmatized or considered distasteful by those around him. He also experienced the pleasure of transgression more acutely than Connor did. Connor tended to accept the violence of the game as simply part of the series' genetic makeup, and described it as zany, predictable, and so ridiculous as to be meaningless, although he did acknowledge that his family or friends might not share this view. He also appeared to derive relatively less pleasure from the transgression and violent play. Instead, his gameplay tended to focus on game elements like resource management and capitalist accumulation, with a focus on accomplishing the "hardest" tasks and collecting status items in the quickest ways possible. He was also keen to participate in online multi-player play, which neither Aaron or I did. The individual play, often focused on narrative and individual exploration was therefore a means to an end for him, which may account for some of his disinterest. However, Mortensen argues that "playing better than others" or playing in order to do well is also a "ethically sound action". ${ }^{217}$ As such, it might be possible to read Connor's approach to the game as being related to the discussion about the role of transgression in GTA, albeit in a round-about way.

217 Mortensen, 159. 


\section{Chapter 5: BDSM, Violence, and Pleasure in the Uncanny Valley}

This chapter is centered on the experience of the uncanny and the fracturing of the self that occurs through dark play. As discussed in the preceding chapter, transgression leads to an erotic experience of continuity, characterized by a dissolution of the self. The sensations we experience as a result of this are often characterized as uncanny. I experienced GTA 5 as a deeply uncanny game because much of the play seemed to point towards deeper psychosexual themes, and transgressive sexual undertones. In this chapter, I will focus on one of my play experiences in particular: GTA 5's infamous torture scene.

In defense of games' violent content, developers and players often claim that games are "just games", unrelated to and thus without power in the real world. However, almost as often, they make appeals to reality, claiming that their environments, characters and violence all reflect reality, and are therefore unremarkable. From a technical standpoint, increased realism is a goal bordering on fetishism.

Using the concept of the uncanny, focused on player experience and the full range of textual interpretations, this chapter will argue that it's more likely that both of these positions are true and that games occupy a liminal position somewhere between the real and the unreal. As Lloyd-Smith points out, texts evade the constraints of the real with impunity; like magic and literature, games "escape the determinants of reality while pretending subjugation to these laws". ${ }^{218}$

218 Lloyd-Smith, Uncanny American Fiction, 10. 
Despite the fact that the uncanny valley in video games is seen as a technical obstacle to overcome, video game play allows players to more easily experience the uncanny in the literary sense, through the doubling and fracturing of the self, and by allowing players to playfully experience repressed desires or anxieties. This means that games will always be uncanny in a Freudian or Bataillian sense. In making this argument, I will connect the concept of the uncanny as a technical consideration to psychoanalytic theorization of the uncanny in literature and explore how games, like print fiction, can provoke the uncanny, paying attention to the particularities of gameplay in this process. I will then explore how uncanniness might augment pleasure and anxieties. Finally, I will explore these concepts through two examples of uncanny scenes in GTA 5 and argue that they are rich with both pleasure and anxiety, both of which are augmented by the concomitant blend of recognition and revulsion that the uncanny provokes.

Based on my authoethography, I argue that in these scenes, players' consciousness seems to hang between that of the victim and the victimizer, a characteristic of successful dark play. Furthermore, maintaining the tensions that characterize dark play in a way that facilitates play requires effort by both the player and the developers. As such, I argue that it is best to understand dark play through a kink lens, wherein the developer is the dominant partner who creates and experience for the submissive player. The submissive player experiences this control and relinquishing of power as cathartic and empowering, but there is a risk that the play is "destroyed" if the delicate balance between tensions is not maintained. 


\section{The Uncanny}

The roboticist Masahiro Mori noticed that in "climbing towards the goal of making robots appear human", our affinity for them increases until there is a dip in positive emotional response to entities that are close to, but not convincingly, human. He named this drop the uncanny valley. ${ }^{219}$ Artificial beings in the valley evoke a range of affective responses, including disgust, revulsion, eeriness, and discomfort. Mori's paper, originally published in the 1970s in a Japanese journal, has garnered attention in the gaming world, where it is of particular interest as a way of understanding the challenges and implications of making video games more realistic, immersive and affective. As such, the uncanny valley has often been framed as a technical problem to be overcome.

In order to increase emotional attachment and immersion for players, designers view aesthetic realism as highly desirable, and they expect that at some point the industry will be able to create digital humans that are so realistic and lifelike that they ascend out of the valley, and take their place alongside living, breathing humans. ${ }^{220}$

The industry and its players understand uncanniness to be the product of a failure in realism, or in programing the minutia of life convincingly, which results in a feeling that something "isn't quite right". For example, researchers have suggested that the feeling of uncanniness results from a lack of forehead and eye movements, which make characters resemble real-world psychopaths whose feigned emotional reactions may lack convincing

\footnotetext{
219 Mori, "The Uncanny Valley [From the Field]."

220 Tinwell, Abdel, and Charlton, "Perception of Psychopathy and the Uncanny Valley in Virtual Characters," 1618.
} 
eye movements. ${ }^{221}$ Game designers have therefore focused on the importance of recreating the eye's incredibly complex and subtle involuntary movements in order to help a player's brain recognize a digital character as human. Game design has certainly made tremendous strides in this direction. The popularization of motion capture, in particular, has begun to close the gulf between digital characters and the humans who provide the model for their movement, expressions and vocalizations

This understanding of the uncanny is similar to Jentsch's, a psychoanalyst who saw the uncanny as something novel, unfamiliar or intellectually uncertain. Jentsch may well have argued that avatars, like the dolls and automata he wrote about, are uncanny because we are uncertain of whether or not they are human. ${ }^{222}$ If we subscribe to this definition of the uncanny, we could overcome the problem of uncanny characters by creating a digital human that was sufficiently realistic or familiar as to convince a rational human that what they were looking at was indeed alive. While it is certainly important to consider the technical aspects of the uncanny, and to advance the digital arts in this way, an understanding of the uncanny that hinges on the unfamiliar is rather simplistic.

At the end of his paper, Mori provides a very brief discussion of why we might experience the uncanny in the way we do. He suggests that the feelings of disgust and eeriness we experience when faced with an uncanny being are linked to our fear of death and our drive for self-preservation, wondering if it is therefore essential to humanness. ${ }^{223}$

\footnotetext{
221 Tinwell, Abdel, and Charlton, "Perception of Psychopathy and the Uncanny Valley in Virtual Characters." 222 Lloyd-Smith, Uncanny American Fiction, 1.

223 Mori, "The Uncanny Valley [From the Field]," 100.
} 
In Mori's assessment "healthy" humans, normally located at the top of the second peak, slip down into the uncanny valley through death. Therefore, corpses and other beings within the valley are reminders of death that provoke eerie or frightening feelings in living. This conceptualization of the uncanny is less about the unfamiliar-after all, we are able to rationalize and are familiar with corpses- and more about the uncanny as connected to fear, death and the dread of "slipping down" from life. ${ }^{224}$ Therefore, I would argue that Mori himself was thinking beyond the technical in his original paper and (perhaps inadvertently) links his definition of the uncanny more to Freud and Bataille than to Jentsch.

An understanding of the uncanny based on familiarity and continuity resembles that of Sigmund Freud, who wrote his essay Unheimlich in part to push back against Jentsch's formulation of the uncanny. Writing against Jentsch's definition of the uncanny as something novel, unfamiliar or intellectually uncertain, Freud argues that the uncanny is in fact a "class of frightening which leads back to what is known of old and long familiar" or a "sinister revelation" of something that is ordinarily concealed from daily human experience and consciousness in the interest of "psychological health and ego stability". ${ }^{225}$ For Freud, uncanny moments are caused by even the briefest recurrence of fears or frights which we have repressed or surmounted. ${ }^{226}$ Unsurprisingly, Freud focuses on infantile fears which should be suppressed, namely the fear of castration. ${ }^{227}$ However,

\footnotetext{
224 Tinwell, Abdel, and Charlton, "Perception of Psychopathy and the Uncanny Valley in Virtual Characters."

225 Freud, "The 'Uncanny,'” 220; Tinwell, Abdel, and Charlton, "Perception of Psychopathy and the Uncanny Valley in Virtual Characters," 1618.

226 Freud, "The 'Uncanny,," 240.

227 Mori's focus on the uncanniness of disembodied hands belies his alignment with Freud in this respect, for the latter the disembodied hand is representative of the fear of castration.
} 
Freud also discusses a second type of uncanniness, instances where "primitive beliefs that have been surmounted seem once more confirmed", such as our "primitive" fear of death which tends to bubble to the surface with even the slightest provocation. ${ }^{228}$ In such moments, the distinction between imagination and reality is effaced and the psychic reality becomes over-accentuated in comparison to our material reality. ${ }^{229}$ The result is a sensation of uncanniness.

The centrality of the fear of death to the uncanny is reminiscent of Bataille's conceptualization of transgression, and the role death plays in eroticism. For Bataille, uncanny feelings are provoked by the move from discontinuity to continuity. Bataille describes discontinuity as a "deep gulf" between beings ("if you die, it is not my death, we are discontinuous beings"). ${ }^{230}$ However, despite this separation, Bataille argues that we can experience the "hypnotizing" draw of the gulf, or death, together. ${ }^{231}$ For discontinuous beings, death (or the experience of the gulf) "means continuity of being", which is an erotic experience. ${ }^{232}$ It is therefore potentially useful to bring Mori's conceptualization of the uncanny valley into alignment with Bataille's understanding of the hypnotic but dangerous gulf that provokes an erotic shift from discontinuity to continuity. If we understand the experience of the uncanny to be an experience where suppressed desires and fears, and especially death, come to the surface, we can also understand the uncanny as moments of eroticism.

\footnotetext{
228 Freud, "The 'Uncanny,'” 242, 248.

229 Lloyd-Smith, Uncanny American Fiction.

230 Bataille, Dalwood, and McCabe, Eroticism, 12.

231 Bataille, Dalwood, and McCabe, 13.

232 Bataille, Dalwood, and McCabe, 13.
} 


\section{The Uncanny in Fiction}

Lloyd-Smith cautions us against getting caught up in Freud's tendency to make assumptions about human development that lead to theoretical dead-ends. ${ }^{233}$ Instead, he uses his book, Uncanny American Fiction, to think about the psychological structures involved in the uncanny. In his argument that all reading of fiction fulfills Freud's requirement for the production of the uncanny, Lloyd-Smith focuses our attention on several "familiar classes" of frightening that are mobilized in uncanny fiction; most importantly for my purposes: the double and death, and the fear of what cannot be spoken. In the remainder of this chapter, I want to demonstrate how the same argument can be made for video games and gameplay. Going further, I will argue that games, due to the particularities of the medium, are especially potent vehicles for the uncanny.

Lloyd-Smith argues that fiction has a peculiarly strong hold over us and is able to move us deeply through the process of identification. He does not mean identification in the sense of a strong affinity for one character, but rather that the fictional world becomes disproportionately and irrationally important to us. We might understand this best in the context of games as being analogous to immersion, or the sense of being fully immersed in the gameworld. He ascribes this to the fact that fiction "happens to us" in a way that is analogous to the "primitive belief" in magic. He argues that as the text simultaneously reveals and conceals what would ordinarily be repressed through the "renegotiation of relations between the self and the world"; the repressed is thereby

\footnotetext{
233 Lloyd-Smith, Uncanny American Fiction, xi.
} 
revived and bubbles up in a way that confirms our "primitive beliefs". ${ }^{234}$ This creates a rich potential for the uncanny in which coincidence or perceived connections between the game world and the 'inner realm of the self (in its unconscious conflicts, fears, dreads)' trigger a reaction in the self, independent of rational justification. ${ }^{235}$

The fact that texts both reveal and conceal, and are both real and unreal is due, at least in part, to the indeterminacy of the relationship between language and object, signifier and signified. ${ }^{236}$ As with texts, everything that is present within the game simultaneously points towards what is absent, and the careful construction of the gameworld by the developers simultaneously creates a "secret geography of the unspoken". ${ }^{237}$ The concept of uncanniness therefore pushes back against media effects research that claims to be able to authoritatively decode what is signified in video game violence and the effects this will have on the real world.

Gameplay augments this dynamic significantly. As an inherently dialogical medium that depends on the player, the act of playing video games is itself meaningful. While different users may approach a game differently, all behaviours allowed and disallowed within a game carry meaning, although this meaning may not align with the authorial intent for the narrative. ${ }^{31}$ Game developer Johnathan Blow refers to this "coagulation of intentional and unintentional meanings" as dynamical meaning, although it is more commonly referred to as ludo-narrative dissonance, in reference to the fact that the narrative or fiction of the game may compete with or controvert the meaning

\footnotetext{
234 Lloyd-Smith, 10.

235 Lloyd-Smith, 159.

236 Lloyd-Smith, 12.

237 Lloyd-Smith, 12.
} 
produced through play, mechanics and rules. ${ }^{32}$ Essentially, interactivity and user input "sabotages" precise delivery of a coherent or sequential narrative. ${ }^{45}$ This dissonance between user and system created when playing games is way of forming a dialogical narrative in which the player can create a cacophony of identities, or "fractured selves". Ludo-narrative dissonance, often framed as a source of considerable angst for game developers in that it can cause failure of communication is also able to create a plurality of possible iterations and meanings. ${ }^{238}$ Lloyd-Smith points out that in uncanny fiction "neither writer nor reader are fully in control, but both encounter the world with hopes and fears as to the outcome...". ${ }^{239}$ Such fictions are spaces charged with meaning between both the reader and author, and between the text and reality. ${ }^{240}$

However, not all destabilizations of meaning result in the production of the uncanny. The production of the uncanny depends "at least in part on an underlying sense of seriousness and even threat". ${ }^{241}$ Therefore, violence holds a particularly important role in terms of the creation of the uncanny in games. It's status as both real/ unreal, serious/ playful, and the way in which players experience it as both something they do and something that happens to them makes it particularly affecting.

\section{The Avatar as the Double: The Cyborg Self.}

\footnotetext{
238 Blow, "Fundamental Conflicts in Contemporary Game Design"; Bogost, How to Talk about Videogames; Lloyd-Smith, Uncanny American Fiction, 12.

239 Lloyd-Smith, Uncanny American Fiction, 11.

240 Lloyd-Smith, 12.

241 Lloyd-Smith, 155.
} 
As with transgression, Lloyd-Smith argues that the uncanny in fiction depends on the "derangement" of both cultural and writerly conventions, but that the abnormal cannot exist without the norm. ${ }^{242}$ One such convention, the act of writing (and playing) "doubles nature, thought, and us". ${ }^{243}$ As an "incarnation" or "embodiment" of one's self, the avatar is a form of doubling, and the relationship between the player and the avatar is perhaps the most important particularity of games when it comes to the creation of the uncanny.

Freud is particularly concerned with the idea of the doubling of the self, or what he describes as the "doubling, dividing and interchanging of the self". ${ }^{244}$ He argued that in the primary narcissism stage of psychic development the double (i.e. our own image in a mirror) begins as insurance against the destruction of the ego: an 'energetic denial of the power of death'. ${ }^{245}$ However, once we have surmounted this impulse the double becomes more sinister: an uncanny "harbinger of death" or a reminder of the base fear that gives power to death. ${ }^{246}$ However, while doubling reminds us of death, it also seems to bring to life something otherwise inanimate, like a text or a gameworld. ${ }^{247}$

As Freud explains, the double is a reminder of a time where the self is not sharply demarcated from the external world and other people. ${ }^{248}$ Therefore, the avatar serves as a double to both ward off death, but also as a reminder of time where the self was continuous. The experience of the self as unfixed, located in a liminal space between our

\footnotetext{
242 Lloyd-Smith, ix.

243 Lloyd-Smith, ix.

244 Freud, "The 'Uncanny,"' 235.

245 Freud, 225.

246 Lloyd-Smith, Uncanny American Fiction, 9.

247 Lloyd-Smith, ix.

248 Freud, "The 'Uncanny,"” 236.
} 
body and our avatar is a source of uncanniness, but it is not related to the ability of an avatar to approximate human-ness accurately, but rather to an assisted return to an infantile desire to remake or extend ourselves which is simultaneously counterbalanced with the understanding of our own inability to do so. Therefore, while the double provokes thoughts of death, it also points towards the potential for continuity (either a time where the self was not demarcated from others, or the experience of death), and this makes engagement with the double a transgressive and erotic experience.

Drawing on Poulet, Lloyd-Smith writes that while the reader consumes the writing, the writing also appropriates the reader. ${ }^{249}$ Poulet describes an "alien self" or double that comes to exist between the reader and their experience of the fiction. While this self is separate from the reader is it also constituted from the reader, or from the reader's possible selves. In other words, the double and the self are co-constituted, and contingent inasmuch as they are separate and distinct from one another. ${ }^{250}$ In video games like Grand Theft Auto the player acts "as a host" to the fiction's "virus" and this facilitates the interchanging of the self with the digital extension of the self. ${ }^{251}$

Whereas Poulet describes this unfixed, liminal self as "alien", I would argue that games act as a cyborg assistive device, allowing us to "[play] the self, and to be an escape artist from the self at the same time" ${ }^{252}$ In much the same way that Lloyd-Smith argues that writing is the production of the uncanny, the game itself forces this escape by setting

\footnotetext{
249 Lloyd-Smith, Uncanny American Fiction, 13.

250 Poulet, "Phenomenology of Reading," 56 quoted in Lloyd-Smith, Uncanny American Fiction.

251 Lloyd-Smith, Uncanny American Fiction, 13.

252 Gannon, "The (Im)Possibilities of Writing the Self-Writing," 484; Derrida and Ewald, "A Certain 'Madness' Must Watch over Thinking."
} 
boundaries, limits and rules. The cyborg self- part digital construct, part real, a blended consciousness shared in the in-betweenness- requires us to engage with the idea that "the self does not exist". ${ }^{253}$ This both artificially limits the expression of the self, and allows us to think about the self differently by dislocating the player's subjectivity from their actions, and allowing players to both be and destabilize themselves simultaneously. ${ }^{254}$ Machine and human must "dance" as the cyborg self, circling the truth but preventing "fixation or conceptualization". ${ }^{255}$ This dance fractures identity and standpoint. ${ }^{256}$ The cyborg self is therefore a possible self, neither truly real nor complete fiction. In this liminal state, any congruence between our real-world fears and anxieties and our experiences within imagined world are made particularly powerful and uncanny. Keogh calls this a "play of bodies", and importantly he emphasizes that this is not only an audiovisual phenomenon. Rather, he argues that this "flickering" between states is corporeally perceived. In bringing the body itself back into the dance between machine and human, Keogh highlights the potential for not only intellectual but embodied pleasure and eroticism through play. ${ }^{257}$

In the last two sections I have attempted to disrupt the idea that the uncanny can be overcome in game design and development through technical innovation. While these advances may certainly reduce the feeling of strangeness that results from doll-like digital humans, the uncanny is inherent to games (and all fictions) as a medium, and therefore

\footnotetext{
253 Fox Harrell and Zhu, "Agency Play: Dimensions of Agency for Interactive Narrative Design," 2.

254 Gannon, "The (Im)Possibilities of Writing the Self-Writing," 489.

255 Gannon, 477; Zita, Body Talk, 204; Cixous, Three Steps on the Ladder of Writing, 6.

256 Fox Harrell and Zhu, "Agency Play: Dimensions of Agency for Interactive Narrative Design"; Haraway, "A Cyborg Manifesto: Science, Technology, and Socialist-Feminism in the Late Twentieth Century."

257 Keogh, A Play of Bodies, 13.
} 
impervious to intervention based on code and design. As Lloyd-Smith explains, the uncanny itself does not exist except as an effect that we can observe best through sidelong looks and out of the corner of our eyes. The uncanny is a "writing effect": the act of writing is itself uncanny. ${ }^{258}$ Similarly, in games, the narrative, rules, as well as the possible dissonance between the two, augment the potential for the uncanny. ${ }^{259}$ Understanding the importance of the uncanny in games helps to step away from essentializing descriptions of the role of violence in video games. Taking this further, the narrative and mechanical potential of the uncanny is often mobilized by game developers in a way that is best understood through a kink-informed lens. In the next section I provide examples from GTA 5 based on my autoethnographic Let's Plays to illustrate how these derangements in form and convention alter the relationship between developer and player and produce an array of possible meaning. By evoking repressed desires, the game provokes deep feelings of pleasure and anxiety.

\section{Case Studies: Torture Scenes}

\section{A. Mr. K}

The first scene I want to discuss is the infamous depiction of torture in GTA 5. In this scene, the playable characters, Trevor, Michael and Franklin, are being coerced by the FIB (the in-game version of the FBI) to do their dirty-work. They are sent to the IAA (in-game version of the $\mathrm{CIA}$ ) to recover a person of interest, Mr. K. In the course of this mission, the player rappels down the side of a building and we see $\mathrm{Mr}$. $\mathrm{K}$ being

\footnotetext{
258 Lloyd-Smith, Uncanny American Fiction, ix.

259 Lloyd-Smith, ix.
} 
interrogated by members of the IAA. A woman, stern-looking and wearing a suit, yells questions at him. As Mr. K insists that he is unable to give her the information that she wants, the woman threatens to rape Mr. K with the flashlight she is holding. She then orders two agents to restrain Mr. K, as she asks "You think you're impenetrable? Let's see how you feel when this [she brandishes the flashlight] is eighteen inches deep". As the player regains control after this cinematic cut-scene and is given instructions to smash through the window behind the agents, we hear the woman say "get the lube. Actually, fuck the lube, let's dry dock him".

This violent scene with explicit sexual overtones is hardly atypical. In playing the game I was shocked at the number of rape threats against men. While this scene is somewhat peculiar in that the individual making the threat is a woman, within the GTA 5 world women take two forms: hyper-hysterical women (or men) who are feminized and depicted as lacking rationality, and cold, stern and severe women who are, essentially, stand-ins for men. In this case, the woman is in charge of the situation, is wearing a suit, and is aggressively wielding a large, black phallus, her apparent sex seems secondary to her masculinization. Given the persistent threat of rape against men by other male-coded individuals throughout the game, I think it's fair to read this situation similarly. Clover makes a similar argument about the nature of the gendered body in horror movies. She argues that male masochism and identification with the female victim relies on an assumption of a "one-sex body", where, as we've seen, maleness or femaleness is determined by the social gendering of acts it performs, undergoes or undertakes. ${ }^{260}$

260 Clover, Men, Women, and Chain Saws, 159. 
Therefore, the IAA agent becomes male through her wielding of a dangerous phallus, whereas Mr. K. becomes female through the potential penetrability of his anus. Conversely, as we will discuss below, Michael regains his masculinity through his willingness to "penetrate" Lazlow. ${ }^{261}$

This implied corporeal spectacle of bodily violation is both sexual in nature, but also designed to be, for a presumed male audience, incompatible with heterosexual psychic organization. The fear of rape here is a fear of transgression where the sexualized human body and images of the wounded, suffering and violated human body are brought together. While the player smashes through the glass, interrupting the potential violence, this brief cut-scene primes the viewer for a mission that takes place shortly afterward.

The mission "By the Book" begins as Trevor and Michael descend a set of stairs, summoned by the FIB agents, and find Mr. K duct-taped to a chair in the cellar. Trevor and Michael have been called in to torture Mr. K (Trevor's role) and to assassinate a target based on the information that he will give them (Michael's role). The player switches between these two roles several times throughout the mission. Despite any ambivalence on the part of the player, Trevor does not hesitate at being asked to conduct the torture, and indeed seems quite happy about it.

The player is made to select a torture device from the table. There are electrical clamps, a jerry-can of water, a huge wrench, and a set of pliers. Rather than move to a cut-scene once the weapon is chosen, the game insists on keeping the players engaged.

\footnotetext{
261 Of course, it could also be read as doubly-degrading to be threatened in this way by a woman. This reading would also require us to rethink the following scene in a way that centers masochism.
} 
For example, if a player chooses to use the electrical clamps, the game requires that the player press two separate buttons to apply the clamps, and another button to spark the clamps. The player then needs to both maintain the pressure on the buttons while monitoring Mr. K's vital signs using an on-screen monitor. As the clamps are applied to Mr. K's chest, he writhes and contorts, and screams while his eyes roll back in his head. During this, the player is required to continually participate by holding the buttons down to continue the flow of electricity.

During the Trevor torture scene, the camera is 'perched' on Trevor's shoulder, giving us a clear view of Mr. K's face and torso. Without seeing Trevor's face, we see him lean his weight into the clamps, and witness Mr. K's back arch and spasm as the electricity passes through him. Each form of torture provides us with a similar view. Trevor can water-board Mr. K: he flips the chair Mr. K is confined to onto its back, leaving the man on his back, legs in the air. The camera follows his face down towards the floor, fixating on the flow of water onto his covered face, as he twists and squirms in an attempt to escape. Throughout this torture, we hear the beeping of the EKG machine: the sound of Mr. K's heart.

The rape threats that precede this scene would certainly be enough to contextualize it as a scene more about rape than torture. However, several pieces of dialogue that are interspersed in the torture serve to underscore this connection. At several points during my play-through Trevor said "oh! I think he's getting a stiffy!" as Mr. K "broke" through torture. This is a clear and deliberate connection between the violated body and the sexualized body, which results in a feeling of eeriness or discomfort due in 
part to the disjuncture between what is being signified (sex or eroticism) and signifiers (torture).

\section{B. Lazlow}

Lazlow is a recurring radio personality character in the GTA series, voiced by American talk show host Jeffrey Crawford "Lazlow" Jones. During the course of GTA 5's narrative Lazlow makes several advances on the playable character Michael's daughter, Tracey. In an earlier interaction, the player uses Michael and Trevor to chase Lazlow down after Tracey appears on his TV show Fame or Shame, where she performs an erotic dance for the camera. Upon catching Lazlow, Trevor demands that he remove his pants. Given Trevor's predilection for threatening sexual violence there is a momentary anxiety about whether or not Trevor intends to sexually assault Lazlow. Instead, after recording a video of Lazlow "dancing sexy", they let him go. While less "dangerous" than the threats made against $\mathrm{Mr}$. $\mathrm{K}$ by the IAA agent, this sort of humiliation still points towards a threat of emasculation through rape.

The next time Michael encounters Lazlow is once again in a context where he is propositioning Tracey, this time for oral sex. Michael attacks Lazlow, using a piercing gun to pierce the DJ's face three times. As Michael pierces Lazlow's eyebrow the submissive man exclaims "ow, are you popping a fucking tent over here? You fucking psycho", suggesting that Lazlow has either perceived or observed that Michael is aroused.

Michael then wrestles Lazlow down and tattoos a penis on his chest or back. Finally, Michael brandishes scissors, and cuts off Lazlow's ponytail. As Michael rips the shirt off of the radio host, he screams "Poppa bear, what's daddy number two going to 
think about this?". This seems to be a reference to Trevor, who Lazlow understood to be Tracey's "second dad" during the initial encounter on the set of Fame or Shame. But the ambiguity of this statement and the manner in which it brings a sexual overtone to the scene enhances the uncanniness of the scene. If the player chooses to tattoo Lazlow's back, the camera is once again perched on the player's shoulder, allowing us to see Lazlow's back as he attempts to escape Michael, who says "don't squirm, just relax". Despite Michael being clothed, this angle certainly conjures images of penetration. Making this connection clearer, as Michael leaves with his children he explains that he knows how to use a tattoo gun because of his time in prison where he learned to "ink his name on his cellie's ass", which we can read as a clear connection between the act of tattooing and violent prison rape.

Yet again, the way the scene is set up along with the implicit sexual nature of the dialogue and interaction create an incongruity of meaning, and a blending of violence and sex. While the scene is largely played for laughs, the incongruity between the actions and what they symbolize result in a profound sense of uncanniness. Despite its violence, the dialogue in the game invites us to interpret this encounter as a sexual one.

\section{Analysis}

\section{A. Violator/Violated: Dark Play and Identification in Liminal Space}

The position of the camera in both scenes, as well as the way it focuses on the reactions and emotions of the victimized men encourages the player to identify with Mr. $\mathrm{K}$ and Lazlow rather than the playable character. This argument is analogous to Carol Clover's thesis that horror films are far more victim-identified than conventional 
understandings of the camera's "mastering, voyeuristic gaze" would have us believe. ${ }^{262}$ As with Clover's argument that rape-revenge horror movies are predicated on the assumption that all viewers, whether male or female, will identify with the raped woman and "feel" her violation, I argue that the player is encouraged to identify and to "feel" the violation of Mr. K. and Lazlow, although they are simultaneously kept tethered to the playable character though the game mechanics. ${ }^{263}$

By making Mr. K's pain so visible, and his internal processes external (especially through the audio feedback we get from the EKG machine), players experience his affect more strongly than Trevor's. Indeed, the auditory signals are especially potent, and I found that these signals of his wellbeing started to blend with my own self-awareness (i.e. his heartbeat began to make me more aware of my own anxiety responses, turning my attention to my own heartbeat). This experience of identification is not about a strong connection to either Mr. K or Trevor, rather it is the sense that the text is happening to the player. In other words, it is a product of the uncanniness of fiction.

What I want to focus on in my analysis is the way in which these scenes are both examples of the uncanny because of the way they leave the player with "the sense we have of turbulent psychological themes just below the rationalistic surface". ${ }^{264}$ It is easy to dismiss these scenes as the glorification of masculine violence, but this explanation doesn't adequately address the feeling of profound uncanniness that both scenes left me with. Lloyd-Smith argues that the ambiguity and uncertainty of these scenes is uncanny in

262 Clover, Men, Women, and Chain Saws, 9.

263 Clover, 159.

264 Lloyd-Smith, Uncanny American Fiction, 30. 
that what appears to be on the surface (in this case, male violence) conceals something below that "remains tantalizingly unspecified". 265

While both of these scenes can be read as examples of fairly extreme violence and fantasies of male power, my autoethnography demonstrates that it is just as possible to understand them as scenes which hint at the uncanny possibility of masochistic pleasure through imagined sexual violation. This dark play is powerful precisely because it allows players to explore "problematic, subversive, controversial, deviant or tasteless" themes and to consider the ethics of their actions. ${ }^{266}$ As I discussed in Chapter 4, dark place forces players to keep multiple tensions active. It is this ability to maintain balance between forces that makes the play pleasurable but also anxiety inducing. It also means that the player shares a meta-awareness between them and their avatar, which I have argued is akin to the movement from discontinuity to continuity, and therefore experienced as erotic.

Following Clover, and based on my autoethnographic observations, I am asserting that the "real work" of this scene is psychosexual and uncanny in that it "turns on the deep excitement generated by the vicarious living-through of the prurient desire for violation, humiliation and sadistic redemption" which must otherwise be "carefully concealed" or repressed. ${ }^{267}$ The game simultaneously causes the player to identify with Mr. K's violation (as I will discuss below), while also actively participating in this violation through their double. The double here becomes the "harbinger of death" through sexual

\footnotetext{
265 Lloyd-Smith, 27.

266 Linderoth and Mortensen, "Dark Play: The Aesthetics of Controversial Playfulness," 5.

267 Clover, Men, Women, and Chain Saws.
} 
violation, threatening to reveal the player's repressed identification with violation, but simultaneously serving as an escape from this identification. In other words, the double both protects the coherent self, while simultaneously "exploding its limits" as the player relishes the "ecstatic suffering" that occurs in the liminal space between player and avatar. This between-ness or "irreducible doubleness" leaves the player in an anxietyprovoking state between truth and non-truth. ${ }^{268}$ As my autoethnographic reflections reveal, I experienced an uncanny fracturing or dissolution of self in these moments, during which my fears and anxieties regarding sexual violation and violence hung somewhere between my consciousness as violator/ rapist and consciousness as violated/raped. This disjuncture "between words and things" produces a space of ambiguity ripe with uncanniness, but also transgression and eroticism. ${ }^{269}$

Lloyd-Smith tells us that not all postmodern destabilizations result in the uncanny, and that the uncanny relies at least in part on an underlying sense of seriousness or threat. ${ }^{270}$ This danger certainly seems to exist in both the scenes discussed, and while it may be premature, I would argue that the relative dearth of discussion among game players about the psychosexual nature of the game reflects an acknowledgment of this potential danger. We might understand this (somewhat uncanny) silence among players using Clover's argument that the "silencing" of horror viewers is due to the "devastating" realization that "one's fellow viewers (or players) are potential rapists" when the player relates to the experience of being raped..$^{271}$

\footnotetext{
268 Bass, "The Double Game: An Introduction."

269 Lloyd-Smith, Uncanny American Fiction, 29.

270 Lloyd-Smith, 155.

${ }^{271}$ Clover, Men, Women, and Chain Saws.
} 


\section{B. BDSM and pushing limits}

For dark play to be pleasurable, the player must be able to maintain a balance between tensions, which includes a balance between fantasy and reality. While dark play "subverts order, dissolves frames, and breaks its own rules", it's also delicate, and the play itself is always in danger of collapsing. ${ }^{272}$ These scenes in particular is a good example of this dynamic, as many players seem to have found that they could not maintain this balance, and that the anxiety the scene provoked overtook any pleasure. This scene, because of the delicate balance that must be maintained between fantasy and reality, is best understood through a kink-centric lens, in order to illuminate the relationship between the developer and the player and the role they both have in maintaining this balance.

There are many online forums and articles discussing By the Book, and I was surprised to find that many are focused on players' negative feelings about the game. My summary of these forums is certainly not quantitative, but the tone of the discussion does seem to be markedly different than discussions of other violent scenes. As we might expect from dark play, the most popular discussion threads about the game are- more than most other online discussions- focused on the ethical and moral implications of this sort of play. Players often point out that the torture scene is no more violent than the game as a whole, while others argue that torture is a more serious offense or that the scene reflects real life more than other parts of the game. However, many players

\footnotetext{
272 Linderoth and Mortensen, "Dark Play: The Aesthetics of Controversial Playfulness," 5.
} 
express that the events of By the Book made them more uncomfortable than anything else in the game, and that they experienced it as truly disquieting rather than as fun. This comment on an article discussing the scene is characteristic of the opinions of these players:

... Playing a game should be fun. GTA was nothing but fun, until this mission, which is a brutal harsh simulation of a reality I don't want to be part of. Not in real life, not simulated. I like movies like 'the Godfather' and 'Scarface', but I don't like pseudo-snuff horror movies with an IMDB-rating of $10 \%$. This is the same thing.... $(\mathrm{ET} 10)^{273}$

ET10's comments are instructive because they point to the breakdown in play that is a risk in dark play. They explain that the scene was a "brutal harsh simulation of reality", indicating that there was a failure for this player in the tension between real and unreal that is necessary to make the scene playful. While they explain that the game had been "nothing but fun" up until this point, the game had certainly had moments of extreme violence preceding this scene. For this player, the issue seemed to be that the scene was not only realistic (which is fun), but nearly a simulation of reality, which pushes the balance too far towards anxiety. This came through in my interview with Aaron, who spoke about in terms of a "social contract" between player and developer. Speaking about what moments provoke anxiety and guilt, he explains:

AARON: ... the strongest guilt and fear emotions are triggered when those sort of auditory profiles [talking about screams and running heard in videos of school shootings] line up in a way and break the boundary.

For him, auditory stimulation was most likely to break the boundary between real and unreal, a division that he explains is critical to the experience of playing a game:

\footnotetext{
273 Amanda Wallace, "Is GTA V's Graphic Torture Scene a Step Too Far."
} 
AARON: ... It's almost like a contract with the game. I want this realistic enough to be fun, but not realistic enough that it becomes real and scary, and real violence.

Importantly, like a contract or a BDSM scene, there is acknowledgement of the play on

both sides. The player and the developer are both active participants in the play, who

each have responsibilities to the other. Aaron succinctly explains this relationship:

AARON: ... I think you sort of sign that contract in your mind when you start playing [...] when you play the game you are saying "I temporarily suspend my conception of what is real for the sake of immersion in this game". It's the willing suspension of disbelief, same thing as when we read books, or watch movies. So, there's an expectation that things will be realistic, but not too realistic, that the game designers will respect some boundaries in that way...

RESEARCHER: So, this idea of a contract, you are suspending disbelief, you are engaging in immersion, and then on the other side you're expecting back this respect of boundaries?

AARON: Yeah, that they won't lead you somewhere [pause] too far from where you've gone before.

Most players expect and desire the developer to push them outside of their comfort zone. Indeed, as I outlined in Chapter 4, doing things they wouldn't do in ordinary life is part of the pleasure of GTA 5. While this developer-imposed constraint is limiting, it may also be pleasurable. Mattie Brice argues that kink is a good framework for reasserting the importance of context in play because it allows us to reconsider the relationship between player and designers, and the meaning of play given that relationship. ${ }^{274}$ For their part, Navarro-Remesal and García Catalán use the concept of "directed freedom" to describe the ways in which player agency and power is limited by a

274 Brice, "Play and Be Real About It: What Games Could Learn From Kink," 78. 
set of rules and constraints that come about because of the authorship of the developer. ${ }^{275}$ While Murray compares the developer's role in this to choreography, we might just as easily imagine the developer as the domme in a BDSM scene. ${ }^{276}$ Drawing from a Hegelian analysis of Master/Slave, Navarro-Remesal and García Catalán argue that the expressive potential of games "comes from the tension between the designers and the players" and between what the designer puts on the table, and how the player reacts and interacts with this. ${ }^{277}$ There is therefore a "voluntary renunciation" of freedom by the player who "consents" to the play experience. This is necessary to create the consensual imbalance of power that allows both a BDSM scene and a game to exist. ${ }^{278}$ As in Studlar's analysis of film audiences, the player takes the role of the masochistic child, who is controlled "within the dynamics of the fantasy". ${ }^{279}$ In both cases, this challenges the focus placed on pleasure through dominance and control rather than submission and masochism. ${ }^{280}$ In other words, it presents an avenue for interrogating the assumption that all violence and transgression in video games is about male power fantasies and never about the relationship between visual pleasure and masochism. ${ }^{281}$

While players expect the developer to precisely manage the experience so that they are not pushed beyond pleasure, the relinquishment of control is also necessary to make this scene pleasurable. Hints of the importance of the dominant/submissive

\footnotetext{
275 Navarro-Remesal and García-Catalán, "Let's Play Master and Servant: BDSM and Directed Freedom in Game Design," 120.

276 Murray, Hamlet on the Holodeck, 153.

277 Navarro-Remesal and García-Catalán, "Let's Play Master and Servant: BDSM and Directed Freedom in Game Design," 122.

278 Navarro-Remesal and García-Catalán, 128.

279 Studlar, "Masochism and the Perverse Pleasures of the Cinema," 270.

280 Studlar, "Masochism and the Perverse Pleasures of the Cinema."

281 Studlar, 269.
} 
relationship between developer and game arise in the online forums, such as this Steam forum where one player asked how to skip the scene and other players respond by questioning the original poster's ability to "get it":

oh my god [Mr. K] doesn't even [fuck]ing die just play the mission or quit the game... its waterboarding, electrifying and pulling teeth. you're not harvesting organs. grow a pair or don't play adult games [... if the original poster] cannot handle the game, he does not need to play it (The Grotesque Abomination) ${ }^{282}$

also its a video game, suck it up. torture has been around for ever and theres nothing that $u$ will be willing to do about it. nothing more than colors and polygons here (Me,Myself,and_I) 283

Players may resent the constraints put on them by the developers, but simultaneously derive pleasure from these constraints. Through their enthusiastic submission, players produce an acting subject who becomes "strong enough" (or in the case of player, good enough or able to "play it right") to "bear the pain and enjoy the pleasures of surrender" to the developer and game. ${ }^{284}$ In making this argument, Crane-Seeber draws on Belkin's analysis of being "man enough" to "take" anal sex, which kinky submissives experience as a mastering of weakness. ${ }^{285}$ Similarly, gamers wear their disaffection as badges of honour, emphasizing their ability to play not only this, but more violent games. This gives players a sense of satisfaction in that they are able to demonstrate their status as players who "get it" because of their ability to understand and engage with the "discipline, protocol, and obedience" that is required to be a good player. ${ }^{286}$

\footnotetext{
282 "How to Skip the Torture Scene?"

283 "How to Skip the Torture Scene?"

${ }^{284}$ Crane-Seeber, "Sexy Warriors," 48.

285 Crane-Seeber, 49.

286 Crane-Seeber, 48.
} 
While the player is often placed into the position of the dominant in the game (logistically this makes the most sense, as gameplay generally requires the player to act, whereas the act of submitting is much more difficult to work into a set of "rules, goals and rewards"), they simultaneously submit to the dominant, the developer(s), who are ultimately compelling the player to act in a certain way in order to succeed. ${ }^{287}$ Anthropy summarizes this by saying that whereas dominants "receive submission", developers "receive play". ${ }^{288}$

However, in case of the two scenes discussed above, the player holds a role as both dominant (as the active participant enacting torture/ psychosexual rape fantasy) and submissive (both through their masochistic identification with the victimized man, and because of the way that the scene requires them to relinquish control to the game and submit to the play experience). This provokes a fracturing of self and a sense of deep uncanniness.

Clover elucidates how a viewer might simultaneously possess the "assaultive gaze" or the gaze associated with controlling the camera and the more painful and feminine gaze associated with the projected screen (or being shown something). ${ }^{289}$ Describing how these two gazes play off one another in Peeping Tom, a film in which a murderous cameraman uses his camera to record and show his victims their own deaths, Hardy explains how this can facilitate a fracturing of the self:

[the film's] structure allows us to watch the image of a film-maker filmically penetrating the object of his desire while he, and we, watch her looking at her

\footnotetext{
287 Navarro-Remesal and García-Catalán, “Let's Play Master and Servant: BDSM and Directed Freedom in Game Design," 131.

288 Brice, "Play and Be Real About It: What Games Could Learn From Kink," 79.

289 Clover, Men, Women, and Chain Saws, 212.
} 
own image, unsettlingly splitting the audience's identification process as we- and he- are both in the position of the aggressor and the victim, at both ends of the sado-masochistic spectrum. ${ }^{290}$

Like Peeping Tom, the torture scenes in GTA 5 don't necessarily do anything unique, rather they draw attention to the symbiotic relationship between the sadism of the filmmaker and the masochism of the spectator by collapsing these two roles and allowing players to occupy both positions simultaneously. ${ }^{291}$

\section{Chapter 5 conclusions: Terrible tensions}

As Crane-Seeber writes of his exploration of the relationship between soldiers and the state, taking kink-centered analysis seriously means "extending feminist and queer analysis to dynamics of pleasure and play that are not remotely politically correct" ${ }^{292} \mathrm{He}$ encourages us to explore the ways people participate in and derive pleasure from their own subjugation, and to explore how play and kink offer the possibility of transforming or re-appropriating traumatic or troubling realities (without falling into the trap of seeing all BDSM as emancipatory). For many, the idea that these torture scenes can be read as not simply male power fantasies, but rape fantasies may be more troubling than simply focusing on physical violence. However, I think that complicating the analysis to look at how players experience both anxiety and pleasure in dark play is useful in disrupting both essentialist textual readings, and simplistic cause-and-effect understandings of how media violence impacts the real world. In this chapter I have attempted to show how the

\footnotetext{
290 Milne, Willemen, and Hardy, The Encyclopedia of Horror Movies, 135 quoted in Clover, Men, Women, and Chain Saws, 178

291 Clover, Men, Women, and Chain Saws, 179.

292 Crane-Seeber, "Sexy Warriors," 51.
} 
nature of videogames makes them an uncanny medium able to help players maintain a series of tensions that gives violence multiple, contingent meanings. The relationship between the player and the developer only augments this. The player experiences power in many overlapping ways in these scenes, and the tension between them and the developer, best understood using a kink-informed lens, helps to maintain the tensions necessary for dark play to succeed. 


\section{Chapter 6: Hood Safari: Digital Blackface and Race Play}

In minstrelsy, a layer of blackness applied to a white face released it from law. Just as entertainers, through or by association with blackface, could render permissible topics that otherwise would have been taboo, so American writers were able to employ an imagined Africanist persona to articulate and imaginatively act out the forbidden in American culture.

- Toni Morrison, Playing in the Dark

The topic of race and racism has been an important theme in at least two of the Grand Theft Auto series; GTA: San Andreas and GTA 5 both include young black men as protagonists, and both make use of tropes about life in urban ghettos, including gang violence, and drug dealing. However, the importance of race in the games seems to have been largely been sublimated by concern about gender and misogyny, outside of a relatively small subset of critical race theorists who have engaged deeply with the series and its depiction of racialized people. ${ }^{293}$

Critical race theorists studying the GTA series including, most prolifically, David Leonard, argue that games scholars tend to take a "colourblind" approach that has excluded racial analysis and denied games' role in maintaining the hegemonic and white supremacist order. ${ }^{294}$ Those scholars who center race in their analysis argue that ghettocentric games create lawless spaces that center stereotypical understandings of

\footnotetext{
293 Leonard, “Not a Hater, Just Keepin' It Real," 83.

294 Leonard, "Young, Black (\& Brown) and Don't Give a Fuck," 248.
} 
blackness and uphold white supremacist and racist ideology. In turn, they materially contribute to state surveillance, control and violence aimed at black communities by reinforcing the idea that black people are dangerous and in need of control. ${ }^{295}$

The dominant tension in the discourse about race tends to be between theorists who see GTA its representations of race as always materially harmful, and those players and developers who see it as "just a game," implying there is no wider social significance to the game. Like radical feminist readings of pornography, critical race scholars center the issue of harm, and especially societal harm, in their analyses. This often essentializes the way these texts are experienced through play and advances simplistic cause-andeffect models for understanding how players interact with games. In finding nuance between these two polarized positions, I will rely on Nash's concept of racial iconography, reading for "ecstasy rather than injury", and suspending normalized readings of GTA in favour of readings which recognize the possibility uncomfortable pleasures within "white-dominated representational economy[ies]". ${ }^{296}$

Nash's work is fundamentally a "critical interrogation of black feminism's approach to representation which treats visual culture, unless produced by black women, as presumptively problematic". ${ }^{297}$ Nash's core focus is on racialized pornography, which she argues is often understood to be especially problematic as it "makes explicit the exploitation" of black female bodies within visual culture more broadly. ${ }^{298}$ Her interest is

\footnotetext{
295 Leonard, "Not a Hater, Just Keepin' It Real," 87; Everett and Watkins, "The Power of Play: The Portrayal and Performance of Race in Video Games," 155.

296 Nash, The Black Body in Ecstasy, 2.

297 Nash, 2.

298 Nash, 2.
} 
in reading pornography in a divergent way, such that she can "avoid a condemnation of the racism imagined to underpin racialized pornography" ${ }^{299}$ Looking at pornography specifically allows Nash to explore how "racial formations" are both constructed and challenged by the performances of pornographic actors and actresses. ${ }^{300}$ This leads her to a critical hermeneutical approach that she calls racial iconography. ${ }^{301}$ This approach shifts away from paranoid reading, or what Nash calls "a preoccupation with injury", and instead "suspends normative readings" to focus on the possibilities of unexpected or subordinate pleasures within phallocentric and white dominated visual economies. ${ }^{302}$ Nash terms these pleasures "ecstasy", which she aligns with jouissance, in an effort to "capture forms of racial-sexual pleasure that have heretofore been unnamed (and some that have been too taboo to name), including blissful performances of hyperbolic racialization and uncomfortable enjoyment in embodied racialization". ${ }^{303}$

Nash approaches pornography as a "rich repository of information about collective fantasy and racial fictions", which she distinguishes from other approaches that tend to either bracket off race entirely or treated racialized pornography as especially dangerous. ${ }^{304}$ As I have argued, this same dichotomous treatment of race has existed in games studies. Nash's approach therefore provides a compelling way forward, by "critiquing the hegemony of particular analytical frameworks, while remaining attentive to the messy relationship between looking and power". ${ }^{305}$ Whereas Nash argues that

\footnotetext{
299 Nash, 2.

300 Nash, 5.

301 Nash, 2.

302 Nash, 2.

303 Nash, 3.

304 Nash, 21

305 Nash, 24.
} 
black porn performers experience pleasure in representations that might otherwise be considered to be minstrelsy; I will argue that a distinct but related set of possibilities are alive in the racialized world of GTA 5. Like Nash, I do not image "pleasure as existing outside of inequality, violence, or pain" or that pleasure "circulates outside of the system of domination which constrain us" ${ }^{306}$ In the same way that kink-centric frameworks have drawn attention to the complicated and contingent ways viewers interact with porn, this chapter aims to destabilize simplistic readings of cross-racial performance and racial pleasure without denying the racism that is present in many video games, including GTA 5.

Importantly, whereas Nash is focused on the experiences of black performers and audiences, my project is centered on the experiences of white players, and how they experience both pleasure and anxiety when playing GTA 5. Therefore, it's impossible to transpose Nash's theories seamlessly onto my own research. Rather, by taking my cues from her, I am attempting to approach game whose meaning is often assumed to be clear and stable from a new angle. Given my own experiences of the game as a white woman, and the experiences of my white interviewees, I also want to explore how "hyperbolic and uncomfortable enjoyment" of racialized simulation is experienced by white players. As such, this chapter is centered on the "paradoxes of pleasure" that are so prevalent in both pornography and video games that rely on race-play. ${ }^{307}$

\footnotetext{
306 Nash, 150.

307 Nash, 3.
} 
This chapter is divided into three main sections. In the first I will provide an overview of the work that has been done on digital blackface in videogames. This important work focuses on the harm done by race-play, which I argue tends to reproduce some of the same analytical pitfalls that have plagued radical feminist's arguments about the societal harm pornography causes. Specifically, critical race theorists tend to focus on providing authoritative analysis of textual representations, and don't place enough emphasis on how meaning is often contingent on play and player experience. Of course, this does not mean that their concern about the potentially harmful impacts is unfounded. However, I am suggesting that their understanding of race-play in videogames is incomplete and often overly simplistic because they do not account for the full range of experiences players have, nor for how they mediate their clashing pleasures and anxieties in a way that doesn't inevitably contribute to increased buy-in to harmful racial ideology. In this section, I will also discuss why games are seen as a particularly harmful medium when it comes to depictions of race.

In the second section, I attempt to show how race-play in video games is connected to race-play pornography. Using autoethnographic reflections, I argue that in both cases, the pleasure in the play is dependent on the recognition and acknowledgement of racial tensions, not their erasure. I believe that pornography scholarship focuses on how race-play can help us to reframe the discussion about race in games, and to help escape the uncomplicated divide between the "self-righteous condemnation" of those who think race-play is uncomplicatedly racist, and the 
"unreflexively libertarian" tendencies of those who see is as just a harmless game. ${ }^{308}$ In the third and final section, I discuss the role of satire in GTA 5, focusing particularly on race humour as a form of race-play that may have the effect of making the real racism that underpins it absurd and laughable.

\section{Digital Blackface}

Despite the well documented scarcity of depictions of blackness in video games broadly, sports and crime/urban video games are dominated by black characters and avatars. ${ }^{309}$ Characterized by their representation of stereotypical blackness, these ghettocentric games are believed to reproduce the ideologies of nineteenth century minstrelsy. ${ }^{310}$ Leonard argues that, like minstrelsy, the embodied fantasies of these games tend to use play to affirm white dominance. ${ }^{311}$ He argues that all representations of urban blackness in games tend to perform this reification of white hegemony, by positioning the ghetto as a war zone and normalizing the connection between people of colour and violence. ${ }^{312}$

But not only violent depictions are examples of digital blackface. Drawing on Elijah Anderson, Leonard argues that depictions of "black coolness" are deeply dependent on white supremacist ideology under which black men are understood as savage and animalistic, and that ghettocentric games amount to minstrelsy performance that allows the players to experience pleasure by taking on a imagined "black body, community or

\footnotetext{
308 Kuzmanovic, "Queer Race Play: Kinky Sex and the Trauma of Racism," 73.

309 Leonard, "Young, Black (\& Brown) and Don't Give a Fuck."

310 Leonard, "High Tech Blackface -- Race, Sports Video Games and Becoming the Other," 1.

311 Leonard, 1.

312 Leonard, "Young, Black (\& Brown) and Don't Give a Fuck," 251.
} 
aesthetic". ${ }^{313}$ Writing about NFL Street, he explains that the game's appeal has less to do with its playability or mechanics, and more to do with its commodification of black street culture and aesthetic including "trash-talking, taunting, showboating, tattoos, earrings, violence and aggressive behavior". ${ }^{314}$

In both these cases, white players derive pleasure from the act of playing blackness. Drawing on hooks and comparing playing videogames to sex tours to Southeast Asia or the Caribbean, Leonard argues that ghettocentric videogames bring "'nasty" unconscious longings about contact with the other" to the surface and allow players to sample the other. ${ }^{315}$ Here, Leonard highlights the implied connection between playing blackface and sexual fantasy and pleasure. It's also possible to connect the unconscious longings he speaks of to the uncanny- and those desires that ought to remain below the surface. Understood this way, it's possible to think of blackface as an example of erotic transgression. The transgressive act is the ability of the player to "try on" blackness, or what Leonard calls "virtual cross dressing". ${ }^{316}$ The player uses a virtual world to "transcend [their own] spatial confinement and ... identity". ${ }^{317}$ They are therefore able to transgress their own racial category, which is a pleasurable but anxietyproducing experience. To be clear, this does not absolve it from its problematic and violent context. As I have discussed, the purpose of blackface has always been to reify and shore up racial categories, even as it permits a temporary porousness. Rather, I mean

\footnotetext{
313 Leonard, "High Tech Blackface -- Race, Sports Video Games and Becoming the Other," 1-2.

314 Leonard, 2.

315 Leonard, "'Live in Your World, Play in Ours': Race, Video Games, and Consuming the Other," 5; hooks, "Eating the Other: Desire and Resistance."

316 Leonard, “Not a Hater, Just Keepin’ It Real," 86.

317 Leonard, 86.
} 
to highlight that the pleasure white players/performers experience is dependent on their acknowledgement (and possibly their belief in) the existence of hegemonic racial ideology.

Traditionally, blackface was a public performance, and its sociality was an important part of its power to reinforce racial order and hegemony. In terms of video games, it's relatively easy to make a connection between this and online play where white players may deliberately take on stereotypical roles as a form of performance for other players. This holds true in multi-player or online games where white players participate in and validate one another's performances. ${ }^{318} \mathrm{~A}$ potent example of this is the staged viral video featuring a World of Warcraft avatar, named Leeroy Jenkins. Created and voiced by a white man, the character ruins one of the game's missions ("raids") through his ineptitude, excusing his actions by proclaiming "At least I have chicken". ${ }^{319}$ Higgin compares Jenkins to the "zip coon" character of minstrelsy shows, a character who, despite attempts to assimilate, ultimately screws things up. ${ }^{320}$ Here, a white man takes on a stereotyped persona and play-acts an established trope of blackness for a group largely comprised of other white people. The video's viral success speaks to the power of this type of performance. The racist overtones of the video were largely ignored or dismissed as harmless fun, highlighting how racism is often normalized in these sort of performative play spaces. ${ }^{321}$

\footnotetext{
318 Nishi, Matias, and Montoya, "Exposing the White Avatar," 468.

319 Higgin, "Blackless Fantasy," 5.

320 Higgin, 5.

321 Higgin, 7.
} 
While nineteenth century minstrelsy and online games share an element of public social performance, my research is focused on solo play. While public performance might be a central characteristic for some GTA play (many players, including my participants, play GTA online as well as alone, or play the game offline in groups), Everett and Watkins help to understand how an analysis of traditional minstrelsy performance can be repurposed in a way that addresses games specifically, including solo play. They are interested in how in-game racial performance, not just portrayals or representations of blackness, mirrors the minstrelsy show. ${ }^{322}$ They deemphasize the role of the audience, and instead focus on the fact that playing is performative regardless of whether someone is watching or not. They argue that there is little difference between traditional blackface which was a means for white men to play out their racialized fantasies within a "safe" performance space dedicated to upholding hegemonic racial ideology, and how players engage with ghettocentric games. Both encourage performers/players to enact the hegemonic racial order through "doing" or performing it within the virtual world, and both are pleasurable for the white performers. ${ }^{323}$

While Everett and Watkins show the similarities between minstrelsy and digital blackface, Leonard takes it further, arguing that games are a uniquely powerful medium for racialized messaging and white supremacist propaganda. ${ }^{324}$ This is in line with Nishi et al, who conclude that games are an ideal medium for the dissemination of cultural

\footnotetext{
322 Everett and Watkins, "The Power of Play: The Portrayal and Performance of Race in Video Games," 149.

${ }^{323}$ Everett and Watkins, 149; Leonard, "'Live in Your World, Play in Ours': Race, Video Games, and Consuming the Other," 4.

324 Leonard, "Young, Black (\& Brown) and Don't Give a Fuck," 252; Leonard, "'Live in Your World, Play in Ours': Race, Video Games, and Consuming the Other," 8.
} 
expectations and assumptions about race. ${ }^{325}$ They identify several factors that make games so powerful: first, they are safe mediums. ${ }^{326}$ By safe, they means that the ability to try on the other through a video game, and to experience the pleasure of the "dangerous, unknown, sexual and unlawful", is without consequence or cost. ${ }^{327}$ As discussed in the previous chapters, my participants explicitly acknowledge that experimentation without consequence is a draw of games, although there are still limits to what they are willing to do. Second, games are immersive, which Leonard argues increases the level of pleasure and excitement, because the player goes beyond observation or voyeurism and becomes the other, able to "live through" racialized fantasies and anxieties. ${ }^{328}$ Third, they are particularly potent education spaces, something that scholars argue is connected to their immersion, and apparent authenticity and realism. In the next subsections I will discuss these concerns.

\section{A. Video games as immersive spaces}

Everett and Watkins make a distinction between mediums that "tell", like TV, and mediums that allow the player to "do" in a dynamic and changing world, like videogames. ${ }^{329}$ Drawing on Prensky, they argue that the more immersive a game is, the more likely a player is to absorb contextual racist information consciously or unconsciously. ${ }^{330}$ Critical race scholars argue that these participatory or immersive

\footnotetext{
325 Nishi, Matias, and Montoya, "Exposing the White Avatar," 465-66.

326 Leonard, "'Live in Your World, Play in Ours': Race, Video Games, and Consuming the Other," 3.

327 Leonard, 5.

328 Leonard, 8.

329 Everett and Watkins, "The Power of Play: The Portrayal and Performance of Race in Video Games," 149.

330 Everett and Watkins, 151.
} 
experiences are pleasurable for white video game creators and players, in part because of their connection to white fantasy and ability to affirm white privilege, and because the pleasurable experimentation and fantasy simultaneously fetishizes and demeans black experience is central to the white player's performance. ${ }^{331}$ This, in effect, turns games into "pleasurable tools for teaching racism [and...] social intolerance" with more "power and allure" than non-immersive mediums. ${ }^{332}$ As discussed, this assumption underlies arguments made about serious games as well: in either case players absorb the messages presented (whether negative or positive) without interpretation or alteration, and these messages are more effectively absorbed because of interactivity and immersion.

However, if we understand this sort of play as dark play then this focus on the message and its real-world impacts, without acknowledging balancing forces, is insufficient. Certainly, this sort of race-play is pleasurable and may play a role in upholding white supremacy, but dark play is counterbalanced with anxiety and confliction. Connected less to outright control and power than to "panic, anxiety, terror and pleasure, dark play is characterized by a player's ability to hold often contradictory feelings in balance with one another. Whereas critical race scholars see this sort of "dangerous and forbidden" play as having clear cut outcomes on the real world, if we understand race-play as a form of dark play, this becomes murkier. Critically, scholarship on dark play shows that players operate in a sort of moral and ethical limbo, suspended

\footnotetext{
331 Leonard, "Not a Hater, Just Keepin' It Real," 86; Nishi, Matias, and Montoya, "Exposing the White Avatar," 464.

332 Leonard, "'Live in Your World, Play in Ours': Race, Video Games, and Consuming the Other," 1; Everett and Watkins, "The Power of Play: The Portrayal and Performance of Race in Video Games," 151.
} 
between real and imaginary worlds. This has implications on both immersion and whether or not we believe games are particularly good teaching tools.

\section{B. Video games as educational spaces}

The state's desire to regulate and control youth's access to video games is connected to their understanding of games as a source of anti-social learning. However, this concern is primarily reserved for young white people, and interventions are aimed at protecting white youth from the perceived influences of ghettocentric games and "gangsta" culture. ${ }^{333}$ Critical race theorists have astutely shown how the call for legal intervention regarding violent videogames serves to uphold racist institutional practices. Leonard draws on Jackson to argue that the legal debates surrounding violence in video games and ghettocentrism, are a form of lynching wherein black bodies are construed as dangerous and in need of state regulation. Following Leonard, it is possible to argue that GTA 5 is such an infamous and contentious game because of its ability to immerse suburban white boys into a ghettocentric environment, which means it is thus seen as a dangerous threat to whiteness, and a source of moral decay, or as Leonard puts it, a "dangerous pollutant". ${ }^{334}$ This concern mirrors social and legal concern about other forms of black culture that are thought to be particularly dangerous or corrupting for white youth. For example, this same dynamic has existed around rap and hip hop. ${ }^{335}$ This anxiety does not extend to black youth; white youth are presumed innocent and thus are

\footnotetext{
333 Leonard, "Young, Black (\& Brown) and Don't Give a Fuck," 249.

334 Leonard, 257, 260.

335 Sullivan, "Rap and Race: It's Got a Nice Beat, but What about the Message?," 607.
} 
able to be corrupted, whereas black youth are at best beyond help, and at worst a corrupting influence themselves. ${ }^{336}$

Games are seen as powerfully corrupting forces because they are considered to be formidable pedagogical tools. ${ }^{337}$ Relating immersion to learning, scholars argue that players do not bracket off the real-world's racism or subvert it. Rather, immersive video games are used as "a filter through which to understand" the real world and the role of race. $^{338}$ They tie this argument to work done by games scholars on learning, who argue that games combine telling and doing in a way that is more effective than traditional classroom teaching and learning. ${ }^{339}$ While critical race scholar's efforts to draw attention to racism in video games is certainly commendable, this argument that media creates cultural meaning in a way that is unmediated by individual agency and experience is not particularly helpful, particularly in the case of games where, as discussed, play is so heavily informed by a host of cultural, social and personal factors.

Everett and Watkins do point out that it is not immersion or the game world itself that is solely responsible for learning. Players bring their own "repertoires of cultural knowledge" to the game, and these inform the meaning they create and derive from the virtual environment. ${ }^{340}$ Resonance between the game world and the racial themes and logics that the player brings to the game from the outside world are what the authors argue make games so pedagogically compelling and effective. ${ }^{341}$

\footnotetext{
336 Leonard, "High Tech Blackface -- Race, Sports Video Games and Becoming the Other," 263.

337 Leonard, "Young, Black (\& Brown) and Don't Give a Fuck," 249.

338 Everett and Watkins, "The Power of Play: The Portrayal and Performance of Race in Video Games," 151.

339 Everett and Watkins, 142.

340 Everett and Watkins, 146.

${ }^{341}$ Everett and Watkins, 146.
} 
Connecting race-play to dark play suggests that the resonance between the ethical frame of the real world and the ethical frame of the virtual world is a way of increasing the affective potential of transgression. In other words, when players recognize similarities between the ethics of the virtual world and the real world, transgressive acts may be more affectively powerful and pleasurable. I am unconvinced that this also means that the game is an effective teacher in the way it's seen to be. As I have discussed in chapter 4, dark play means players are unable to suspend disbelief. Instead, their recognition of the un-real realness of the virtual world encourages complex negotiation and mediation between in-game and real-world ethics. This seems to challenge the idea that games teach players a uniformly harmful ideology. On the other hand, transgressive play is only transgressive if the player recognizes the taboo.

Therefore, if a player fails to recognize the transgressive dynamic of race-play, they will not experience the game as dark play. In such instances the game may very well confirm all of the beliefs.

\section{Glorification, reality and authenticity}

Drawing on King and Krzywinska, Everett and Watkins argue that video game history has been characterized by a focus on increased realism and authenticity, which requires not only greater graphics or characters, but the ability to "capture the cultural sensibilities of a particular racial or ethnic group's world experience". ${ }^{342}$ Monson argues that the presence of racist stereotypes in games helps to construct a sense of authenticity, by

\footnotetext{
342 Everett and Watkins, 144.
} 
aligning the virtual world with the dynamics of the "real" (race-based) world. ${ }^{343}$ This

means that games go beyond play an create a tangible simulation where white

supremacist ideology and structures can be reproduced. ${ }^{344}$

The clearest example of how authenticity can be mobilized in the service of racist

ideology is in a discussion of GTA 5 on Stormfront, a white nationalist and neo-Nazi

webpage. While such a discussion is perhaps not representative of most players'

attitudes, it is still telling. Here, one Stormfront forum user chides the others for the ir

excitement about its release:

What is it with all the GTA posts on this [site] lately? In my opinion [white nationalists] should not play this game. It's a THUG game. It's aimed at thugs and thugs wannabee's and the mayority (sic) of people who plays this game are black thugs. Playing this game is worse then (sic) listening to rap music. (lex2012) $)^{345}$

Similar discussions occur throughout a forum discussing the release of GTA 4, a game set in New York City, with a white Eastern European protagonist. Here, Stormfront forum members once again discuss whether or not the new protagonist is "white enough", and discuss whether or not they played GTA 3 despite its "glorification" of blackness:

Although GTA [San Andreas] can be looked at as promoting black culture, part of the games appeal is it's (sic) realism, and San Andreas's main characters are mainly black, gun-toting, drug pushing losers... (shorter) ${ }^{346}$

I didn't think the game [GTA 3] promoted black culture, whatever that is. Just showed the reality. Which is effectively all the black characters were low life scum with no prospects. Maybe that is black culture. (blue ice) ${ }^{347}$

As far as "glorifying" blacks, to me [GTA 3] didn't do anything but further degrade them. In most of their "epic" movies and "music" they have to look at as their

\footnotetext{
343 Monson, "Race-Based Fantasy Realm," 53 quoted in Nishi, Matias, and Montoya, "Exposing the White Avatar," 468.

344 Leonard, "Live in Your World, Play in Ours': Race, Video Games, and Consuming the Other," 3.

345 "GTA V - Stormfront."

346 "Stormfront - GTA IV."

347 "Stormfront - GTA IV."
} 
pride and "culture" are usually about pathetic drug addicts and sex-obsessed, foul mouthed murderers in a slum setting. Most take this in with pride, and actually reject anything above this as "not black enough". [...] I guess playing through the game re-enforced my pre-existing prejudices -- that blacks make their own hell through something corrupt or inferior in their nature. (Ragnarokalypse) $)^{348}$

The Stormfront forum users broadly agreed that the media is oversaturated with glorified black culture. While expressing dismay at this, they simultaneously argue that these depictions ultimately reveal the "truth" or the "reality" about black America: that black communities and black people are a pestilent danger to whiteness. This may lend credence to Leonard's argument that the GTA series is simultaneously able to claim that it is celebrating blackness, while actually reinforcing negative stereotypes about black communities when it is interpreted as showing authentic black experience. If we accept this perspective, the result of claiming realism and authenticity is that the game becomes an "effective [and...] uncontested" teaching devices. ${ }^{349}$ Similarly, hooks explains that fucking (or in this case playing) is a way of "confront[ing] the Other, through which white people both dominate the Other and remake themselves in order to "leave behind white innocence" and "enter the world of experience". ${ }^{350}$ For example, the built environment of the game helps to uphold ideas about certain neighborhoods and communities. The dilapidated buildings, petty crime being conducted in alleyways, trash-filled street, and soundscape which might include gunfire and sirens, designed to give black inner-city neighbourhoods "colour" within GTA 5 reinforce understandings of predominantly-black

\footnotetext{
348 "Stormfront - GTA IV."

349 Everett and Watkins, "The Power of Play: The Portrayal and Performance of Race in Video Games," 145.

350 hooks, "Eating the Other: Desire and Resistance," 368.
} 
neighbourhoods as deviant, different and dangerous, while allowing any criticism to be dismissed on the basis that it is an authentic representation. ${ }^{351}$

However, as I've suggested, these players are not engaged in dark play, which requires the recognition and acknowledgement of the taboo. Neo-Nazi players most likely do not see the race-play in GTA 5 as being transgressive because they collapse reality and fantasy entirely. Further, we might also read this conversation as an example of an unconscious attempt to ward off or disavow their own desire for Black culture. In this reading, there is a deliberate rejection of dark play through a denial of any pleasure in blackness and black culture.

While the experiences of all players should be contextualized within a culture and society imbued with white supremacist values and ideologies, not all players are the sort of active white-supremacists these Stormfront users are. For many of these players, while they undoubtedly harbour prejudice, the depiction of race in GTA is likely recognized as "problematic, subversive, controversial, deviant or tasteless". On the other hand, Stormfront users appear to take these depictions as authentic and realistic, and therefore as not taboo and not transgressive. Dark play requires both an understanding that these views are stereotypical, and an understanding of the power of the racial ideology that underwrites them. Further, understanding this as race-play means that we must complicate the idea that these representations are taken up as authentic depictions of blackness by players, because race-play often limits player's ability to suspend disbelief,

\footnotetext{
${ }^{351}$ Everett and Watkins, "The Power of Play: The Portrayal and Performance of Race in Video Games," 146.
} 
and may increase their ability to think complexly about morally and ethically thorny media.

\section{Racial iconography and race play}

Leonard pushes back against discourses which aim to position the fetishization of urban blackness as progressive or emancipatory. ${ }^{352}$ This is a useful reminder throughout this thesis as it warns us against repeating some of the polarized and possibly hyperbolized rhetoric about pornography that was circulated during the Sex Wars, and highlights the complexity of holding onto an understanding of pleasure, oppression and violence simultaneously. Leonard is interested in study in videogames for moments of what Eric Lott calls "racial insult and racial envy", a seemingly contradictory mélange of contempt and desire. ${ }^{353}$

While Leonard argues that it is important to understand what deriving pleasure from race and the racialized other means, his discussion of the particularities of this pleasure is relatively sparse. This may be because centering pleasure can often be construed as an attempt to dismiss troubling aspects of the game, or as an overemphasis on the positive experiences of some players. However, as I have attempted to show, dark or transgressive play requires us to engage with uncomfortable pleasures and to understand how players hold pleasure and anxiety simultaneously.

Jennifer Nash undertakes such a project in her book on black ecstasy in pornography. Like her, I have no interest in exculpating GTA or any other ghettocentric

\footnotetext{
352 Leonard, "Young, Black (\& Brown) and Don't Give a Fuck," 249.

353 Leonard, "High Tech Blackface -- Race, Sports Video Games and Becoming the Other," 1.
} 
game. Rather, I believe that her interpretative framework, which emphasizes the complexity of pleasure, even when it is uncomfortable or unnerving, is a much-needed angle of approach to games studies. Scholars who study race in pornography, including Nash, are often more able to hold onto these contradictions and uncomfortable tensions. As such, I believe that there is a considerable advantage in relying heavily on pornography studies in an attempt to complicate how we understand race play in video games.

Additionally, by opening the door to porn studies, we also make room for kinkcentric analyses. In much the same way that kink can be used as a framework to understand the complex and often contradictory nature of violence and sexuality, the pleasure of racial performance as seen through the lens of kink and BDSM can help us to understand the complex tension between power, pleasure and anxiety that players navigate. In the following section I will attempt to show how understanding race play porn and racialized kink practice can help us to read GTA 5 and other ghettocentric depictions of blackness in games in a way that accounts for uncomfortable pleasures, anxieties and ambiguities.

\section{A. Race play porn and the blurring of fantasy and reality}

In their exploration of race-play porn, Smith and Luykx address the tension between pornography's potential as a repressive medium, and its ability to be simultaneously freeing. They argue that race-play porn offers opportunities for excitement and titillation that uphold white supremacy while simultaneously providing 
opportunities for marginalized people to disrupt or play with these systems. ${ }^{354}$ The authors examine race-play BDSM scenes that draw on historical realities (i.e. slave auctions, Holocaust scenarios) because these scenes purposefully blend reality and fantasy. This is a tactic games use as well. In games, as in race-play BDSM, the appeal of the scene is in its resemblance to reality in as much as it is in its wanton fantasy. Of course, remembering that participants often want the game to be "as realistic as possible" without being "too real", balance is key.

In race play porn, the black/white pairings and the submission/dominance dynamics don't attempt to erase or obfuscate painful racial and ethnic histories and traumas. Instead, this pain, trauma and authentic affect underpins the erotic charge of the scene and make the fantasy more believable. ${ }^{355}$ The clear acknowledgement and centering of the experience of racism is, as hooks suggests, the only way to make an encounter between white and black people happen in a way that is not based on "denial and fantasy". ${ }^{356}$ Simply put, pleasure is derived from the purposeful and explicit connection to violent realities, but because of this can potentially avoid the violence of intimate contact with the other that ignores or erases racial domination. ${ }^{357}$

While there is a connection between reality and fantasy, this is not to say that participants transpose the dynamics of their race-play into their real lives. Authenticity here is less about action in the real world, and more about capturing authentic affect in the service of a particular erotic encounter. This is similar to the way that GTA uses it's

\footnotetext{
354 Smith and Luykx, "Race Play in BDSM Porn," 433-34.

355 Smith and Luykx, 434.

356 hooks, "Eating the Other: Desire and Resistance," 371.

357 Smith and Luykx, "Race Play in BDSM Porn," 433; hooks, "Eating the Other: Desire and Resistance," 371.
} 
realistic gameworld to create a porous boundary between real and unreal. GTA 5's

affective power requires the player to recognize and be fluent in the racial dynamics and symbology that connect San Andreas to its real-world counterpart. The game expects that players are bringing an understanding of racial dynamics to the scene, without which much of the emotional potential would be lost. The game is not a one-way meaning device- rather, it is attempting to work within players' cultural frames to poke and prod at anxieties and pleasures.

In their analysis of the pornographic film Niggas' Revenge, Smith and Luykx emphasize the importance of blurring reality and fantasy for BDSM. The film centers on the Blake brothers, three black men who are being harassed by their white neo-Nazi neighbours. The Blake brothers capture the white men and retaliate, raping and degrading them. Smith and Luykx argue that the film can be read as being both a piece of performance art about racial revenge and as a documentary about interracial sex. ${ }^{358}$ This tension between performance and documentary, and fantasy and reality, is what makes the film erotically powerful. ${ }^{359}$ However, this balance is tenuous and delicate. As with all dark play, the balance can be upset, and the play is constantly at risk of being undone. The authors describe how one of the black leads, Bobby Blake, embraces his role as the epitome of hypersexual, animalistic blackness so convincingly that he risks "awakening the fears" of white viewers, and diffusing the erotic power of the scene. ${ }^{360}$

\footnotetext{
358 Smith and Luykx, "Race Play in BDSM Porn," 439

359 Smith and Luykx, 439.

360 Smith and Luykx, 442.
} 
My autoethnographic reflections on the game suggest that the racial

performance in GTA is similarly characterized by a set of tensions that must be held in balance in order to maintain its playfulness and transgressive eroticism. These "tantalizing performances" are, as Stuart Hall insists, neither entirely positive or entirely negative. ${ }^{361}$ Writing about queer minstrels, Daniel explains that they are "slippery subjects" that both "ruin and rely on the criteria of the "fake" and the "real"', that are most productive in moments of illegibility, unreliability. ${ }^{362}$ They "exemplary antisubjects", both painful and pleasurable in part because rather than avoiding racism, games like GTA 5 embrace and appropriate it, demonstrating how racial hierarchies can be simultaneously subverted and reinforced (even within the same moment) depending on the player's own experiences (including, of course, their race)..$^{363}$ I found that encountering (or identifying with) this sort of anti-subject provokes a sense of deep discomfort or uncertainty because it interrupts the safety of understanding and induces "embarrassment, doubt, shame, [and] a state of intensity with regard to your position that is the bitter opposite of knowledge". ${ }^{364}$ This balance might be achieved differently for different players. I think it is reasonable to assume that black players may experience the same counter-hegemonic function that Smith and Luykx discuss when playing ghettocentric games, although more research would be required to confirm this. However, given the scope of my research, I will focus on white players and will attempt to elucidate how players manage these tensions in order to keep the play playful. In

\footnotetext{
361 Hall, "What Is This 'Black'in Black Popular Culture?"

362 Daniel, "'Why Be Something That You're Not?," 14, 23.

363 Smith and Luykx, "Race Play in BDSM Porn," 443-44; Daniel, ""Why Be Something That You're Not?," 23.

364 Daniel, "'Why Be Something That You're Not?," 24.
} 
doing so, I want to make a clear connection between the affective experiences of watching race-play porn and the racial performance players engage in when playing GTA 5.

\section{GTA 5 as race-play}

GTA 5's black protagonist is Franklin, a young man who has recently been released from prison and is living with his aunt while he tries to make a legitimate living. When we meet him, he is working as a car repossession contractor. Initially surrounded by a cast of black characters, Franklin meets Michael, and subsequently Trevor, and branches out into white-collar crime and a much whiter social circle. Playing as Franklin is an aesthetically appealing experience. Everything from his dress, the quick banter back and forth with his friends, and the pleasure of getting in his car or motorcycle and turning the radio to one of GTA 5's celebrated hip hop radio stations is an enjoyable embodied experience. That being said, it is not without anxiety. I enjoyed playing as Franklin, but this pleasure was always couched in an underlying discomfort:

Race is on my mind constantly when playing this game. Franklin's walk, dress, language use, etc. all puts his race front and centre to me. For me, I think this is a big reason I don't identity AS him and am even perhaps a bit uncomfortable identifying with him

My interviewee had an analogous relationship with Franklin. He explains that while he does not see himself reflected in any one character (based on age, race and class), he does identify with Franklin's struggle to improve his life:

AARON: ... Franklin, I find his storyline really engaging as well. I think...it's... in terms of social commentary it's one of the best of the game. Because it's that sort of struggle for someone to improve themselves when they've sort of been dealt a bad hand in life... I relate to Franklin because of the adversity he faces, right, so 
it's easier to sympathize with him because he's not like...ah.. like a bored old guy who gets back in the game for money, he's trying to better himself and he can't really find a way to do that.

Franklin is both relatable and fulfills the stereotypical role of the ghetto protagonist.

Whereas Trevor allows players to explore violent and chaotic play, Franklin's character provides white players with a compelling character arc, as well as an experience of crossracial play, provided the player is fluent in the racial tropes the narrative draws on.

Despite being one of the more relatable characters and having a stronger moral compass and more common sense than either Michael or Trevor, Franklin's blackness also seems to be his undoing. In my field notes, I write:

Franklin seems to act like the [trope of the] black man. He really can't do anything right, all his missions [...] end in him over his head and often embroiled in violence (failed kidnappings, failed drug deals). His association with other black men appears to be leading to his ruin.

In these excerpts I think it's possible to see that player experience of race-play is informed by pleasure, and identification, but also by anxiety and recognition of racial tropes and stereotypes. A potent example of how these things come together is how, soon after the game was released, some players began to discuss whether the police in the game were more likely to attack and kill Franklin on the basis of his race. ${ }^{365}$ Rockstar denied this, saying: "This is absolutely false, the in-game police don't treat one lead character any differently from the others". ${ }^{366}$

\footnotetext{
365 The perception that black characters were treated differently went beyond the police. In their coverage of the issue, in an article on the issue Forbes quoted players who believe that the white women in the game are also racist: "White chicks in GTA $V$ are RACIST! they call the cops and run away from Franklin just because he's black [...]". Thier, Dave. "Rockstar: 'GTA 5' Cops Not Racist." Forbes. Accessed March 11, 2018. https://www.forbes.com/sites/davidthier/2013/09/27/rockstar-gta-5-cops-not-racist/. 366 Bernstein, "The Franklin Conspiracy."
} 
Drawing on Foucault, Farley argues that, like sex, race is an "especially dense transfer point for relations of power" ${ }^{\prime 367} \mathrm{He}$ is mostly concerned with the sadomasochistic nature of race in society and argues that "race is the preeminent pleasure of our time". Here, whiteness is not an identity or a colour, but is a way of feeling pleasure about one's body in a way that depends on the positioning of the black body as fetish object. ${ }^{368}$ In this way, whiteness is the "sadistic insistence" on the illusion of race and of blackness as a masochistic complicity [...] these two pleasures, one sadistic and the other masochistic, meet each other in the production of the "Truth" of race". 369 The colourline, he argues, depends on these three dynamics- pleasure, humiliation, and colourblind denial- for its maintenance. ${ }^{370}$

Farley describes the "wake of a black man on [...the] street" as a sensual space in which people react around him in response to his blackness (for example, the common experience of being followed around a store). ${ }^{371}$ This a form of race pleasure because it both results in pleasure on the part of the white bystander who can frame the black body as potentially dangerous, and is a form of humiliation visited on the black body. The example of race-play I describe above shows how players simultaneously embody both of these roles (however tenuously). Players are both able to experience a sort of masochistic humiliation at the idea that Franklin would be unjustly targeted by the police (redoubled by their own outrage at this humiliation which demonstrates their ability to

\footnotetext{
367 Foucault, The History of Sexuality: An Introduction, Volume I., 103 quoted in Farley, "The Black Body as Fetish Object," 467.

368 Farley, "The Black Body as Fetish Object," 458, 461.

369 Farley, 458, 469.

370 Farley, 481.

371 Farley, 489.
} 
"get it") while simultaneously generating pleasure in their own whiteness through their acknowledgement and understanding of this racial dynamic to begin with.

A small group of players eventually set up an experiment in an attempt to test the hypothesis that the police treat Franklin differently. ${ }^{372}$ While hardly scientific, their results do mainly confirm what the developers have said: that other characters experience as much attention from the police as Franklin does. What is important here is not necessarily whether or not this is true, so much as it is what players expect to experience, and that their outrage about this and sincere belief that their avatar was being discriminated against reveals both pleasure and anxiety in their embodied racial performance.

Importantly, racial pleasure can be obtained directly, or textually. Farley argues that the textual pleasure of "talking, reading, writing, or thinking [about blackness]" reproduces blackness in its tendency to "lavishly decorat[e] the black body with statistics, stories and images of violence, narcotics, illiteracy, illegitimacy and disease". ${ }^{373}$ I think it's possible to argue that the experience of white players who believed they were being unfairly targeted by the police is a form of race pleasure where the black body is used as a fetish object onto which harm is amassed and which white players experience as a sort of pleasurable re-creation of whiteness as a category that is "not-blackness". However, here it is important to make a distinction between race pleasure (Farley's term referring

\footnotetext{
372 Hernandez, "Someone Actually Tried Testing Out If GTA V Cops Are 'Racist."”

373 Farley, "The Black Body as Fetish Object," 475.
} 
to the sadomasochistic character of the colourline) and race play (the deliberate employment of race in sexual pleasure).

Kuzmanovic argues that race play often originates in true racism and racist feelings, but that it allows for a controlled, acknowledged and ritualized expression of these feelings that might have positive psychological and social results. ${ }^{374}$ But most importantly, unlike racial pleasure which relies on colourblind denial of these feelings and attitudes, race-play require a mutual and forthcoming acknowledgement of the relationship between "inter-racial desire and racial bias and stereotyping". ${ }^{375}$

GTA 5's affective power depends on the recognition and acknowledgement of its racial logic. Therefore, while it may be an example of race pleasure (which would mirror Leonard's arguments about the game as an instrument of white supremacy), it is perhaps better understood through a kink-informed lens as a form of race play where there is a mutual acknowledgement of both the importance of race and the pleasure taken in race on the part of the player and developer.

\section{A. White desire and race play}

Franklin is coded as sexual in a number of ways. His is fit, muscular and well dressed, especially when compared to the two middle-aged white characters. The first explicitly sexual experience is an encounter at a strip club where the game requires a player, playing as Franklin, to participate in a mini-game style lap dance in which you

\footnotetext{
374 Kuzmanovic, "Queer Race Play: Kinky Sex and the Trauma of Racism," 73.

375 Kuzmanovic, 79.
} 
attempt to fondle the white dancer without the bouncer noticing. Players can also press a button to have Franklin flirt with the dancer. If they are successful enough, the woman will go home and have sex with them. Thereafter, Franklin is (in my play experience) the only character who is depicted leaving strip clubs in the short cut-scenes that play when switching between characters, although all characters are able to visit the clubs. The game provides ample opportunity for any of the three protagonists to engage in this behaviour, although it is telling that the interactive sexual elements are introduced through Franklin: he is both the player's introduction to and reminder of the possibility of sexual titillation in the game.

In this context, a white player (especially in first person) is able to perform a sexualized blackness. They are encouraged to take Franklin's point of view in observing and touching the dancer, and their input and success at playing the game can potentially confirm his sexual prowess. Of course, the player can then individually choose to continue performing race in this way, by visiting strip clubs or sex workers. Future research on sexuality and race play within the game that focuses on which character players use as avatars when having sex with sex workers would be interesting. Given players have the option of using a first-person view in these scenes may be an example of the game being used to explore embodied race-play or cross-race fantasies.

Given that race play requires an acknowledgement of how racial tensions will be employed from both the player and the developer, it's worth coming back to the idea that the developer and player are engaged in a dominant/submissive relationship in the context of the gameplay. As I discussed in Chapter 4, there is a voluntary renunciation of 
freedom on the part of the player, who is asked to consensually react within the confines the dominant designer has given them. There is therefore a consensual imbalance of power in gameplay, as there is in a BDSM scene. ${ }^{376}$ This adds complicated layers to the racial performance of GTA 5. Players are asked to engage in race play and are expected to perform well in the role they are given. When this role is playing as a young black man, playing well may require players to play within the acknowledged racial dynamics of the scene; this sort of play might therefore be experienced as submission to the game's logic and the developer's design, but also as a mastery of the character and a chance to play well.

A number of scholars link race and BDSM in a way that expounds the connection between race play, eroticism, and GTA 5's black protagonist. Drawing on Stoller, Smith and Luykx argue that both BDSM and race relations in the USA share a range of affect that includes anger, cruelty, humiliation, and revenge, and that both are heavily informed by both eroticism and violence. ${ }^{377}$ Therefore, despite the relative scarcity of explicitly sexual content in GTA 5, the existence of a black protagonist, who white players are able to control and live vicariously through, is in many ways marked by a comparable racial history and charged with the same sexual energy as race play porn. This is especially true given the psychosexual nature of the game, as well as the explicit homosexual undertones of the characters' relationships.

\footnotetext{
376 Navarro-Remesal and García-Catalán, "Let's Play Master and Servant: BDSM and Directed Freedom in Game Design," 128.

377 Smith and Luykx, "Race Play in BDSM Porn," 436; Stoller, Observing the Erotic Imagination.
} 
My apprehension about Franklin as a character who was pleasurable to play was largely rooted in my own understanding about the ways in which white women take pleasure in the imagined hypersexuality of black men. I didn't like playing the scenes where Franklin was sexualized, and actually skipped the club scene about half way through. In part, I've never found pixelated ladies all that titillating, but the experience was also unsettling. This isn't to say it wasn't pleasurable, but that for me in those moments the anxiety associated with the racial performance upended the balance that should make dark play enjoyable.

Throughout my autoethnographic analysis, I observe that play moments like this often felt like sex scenes between me and the developers, with Franklin in the middle. Playing a black character in this context felt loaded with cultural meaning. As a white woman who has thought about how my own relationship to black men's sexuality is heavily informed by racism and stereotypes, the scene was uncomfortable and made me feel a certain degree of shame. I don't know that the scene would have been any more pleasurable with any of the other characters but playing with a white character certainly would not have provoked the same sort of fracturing of self, wherein I had to hold my own desire and relationship to interracial sexuality and my cultural and social knowledge in tension with one another. In this moment, any pleasure was to be found not in the sexuality itself per say, but in my ability to recognize the racial ideology at play, to be "in the know", and to hold any pleasure in balance with the anxiety the scene provoked. 


\section{B. Satire and Realism}

In this section, I intend to elaborate on the connection between satire and race humour and demonstrate how satire is related to dark play in that it pushes back against essentializing interpretations and requires players to hold multiple ideas in tension with one another. Humour and satire are crucial elements of the GTA series, and one that is often focused on race. However, if one were to read only the critical race theorists' accounts of the game, it would be possible to come away without an understanding of the overarching importance of satire and humour to the game. This is understandable, much like the refrain "it's just a game", "it's just a joke" is often used to dismiss the problematic aspects of the game. But because satire is a defining feature of San Andreas, it informs how the player experiences all moments within the game, and it is therefore imperative to understand GTA 5 as a text without taking its nature as a satirical piece of work into account.

The tenuous connection between reality and virtual is important to the success of satire. As Mulkay writes: "it is precisely the symbolic separation from the realm of serious action that enables social actors to use humour for serious purposes". ${ }^{378}$ This hints towards the ways humour and satire are connected to transgression and dark play. In dark play, players can occupy a liminal space between real/unreal. This allows them to explore the pleasurable tension between conflicting affects. Humour seems to serve a similar function. It allows players to simultaneously escape the real, while paradoxically engaging with serious issues.

378 Mulkay, On Humor: Its Nature and Its Place in Modern Society, 1. 
Satire is a slippery concept, and many scholars have written about the inherent difficulties in defining it outside of the narrow definition of classic Roman Satire. All seem to agree that it is essentially impossible to define satire using either a set of formal characteristics (as is done with Roman Satire) or only it's "informing purposes" or authorial intent. ${ }^{379}$ There is also broad agreement that defining satire as a bounded literary genre is insufficient, especially given developments in visual and digital media. ${ }^{380}$ Beyond that, defining satire can sometimes feel like an exercise in defining the pornographic: "I'll know it when I see it". This is important in the context of the GTA series because of the central role of humour, and particularly racialized humour in the game.

Phiddian argues that satire is not a strictly literary form, but rather that is it a rhetorical strategy that can be taken up by a wide variety of mediums. The strategy relies on "wittily provoking an emotional and intellectual reaction" on an important issue. ${ }^{381}$ This, of course, does not mean that the audience has to agree on what the purpose of the text is, or reach an "accurate" understanding of it, but rather that it is simply understood as "making a point". ${ }^{382}$ In addition, humour, or at the very least wit, is often considered to be a fundamental component of satire. But a sense of moral seriousness is also necessary, and these two characteristics often seem to work against one another. Condren argues that that if we overemphasize the role of moral seriousness, we eliminate the importance of humour. And vice versa: "to locate [satire] in the non-serious

\footnotetext{
379 Condren, "Satire and Definition," 375.

380 Condren, 376.

381 Phiddian, "Satire and the Limits of Literary Theories," 44.

382 Phiddian, 49.
} 
presupposes humor to be essential, and ethical critique superfluous". ${ }^{383}$ He concludes that satire is often ambiguous, and "transgresses any bifurcation between serious and non-serious, drawing its power from shifting between or combining the two". ${ }^{384}$ This is important given my focus on dark play and transgression. Like in those concepts, satire derives its power through its ability to balance seemingly contradictory affects.

There seems to be little doubt among reviews, gamers, and journalists that GTA is satire in this regard. Celebration of GTA 5's satirical success is apparent in many reviews of the game. For example:

[GTA 5 is] pulverising, nihilistic satire on western society. Reality TV, celebrity magazines, social media, plastic surgery, pop psychology books - all get savaged via the often hilarious commercials on the game's many radio and TV stations. ${ }^{385}$

Rockstar's insatiable appetite for satire and parody permeates every corner of GTA V, and their strike rate shames even the sharpest sectors of more mainstream media." 386

However, drawing on Marshall, Phiddian calls attention to what he sees as the problem of criticism of satire: the idea that the "standards of judgement are indubitable", or that the reader can be sure of either the author's attitude is or what the author expects or desires the attitude of the reader to be ${ }^{387} \mathrm{He}$ explains that attempting to evaluate satire using a universal standard of taste, "moral and hermenutical unity", or an authoritative interpretation is not possible. ${ }^{388}$ Despite this, Phiddian is certain of the importance of the author, because "to call something satirical is to imply a satirist", even

\footnotetext{
383 Condren, "Satire and Definition," 388.

384 Condren, 388.

385 Stuart, "GTA 5 Review."

386 Silver, "GTA 5 Review."

387 Marshall, "Gulliver, Gulliveriana, and the Problem of Swiftian Satire" quoted in Phiddian, "Satire and the Limits of Literary Theories," 48.

388 Phiddian, "Satire and the Limits of Literary Theories," 48.
} 
if we may not be able to authoritatively know what their intent is. This means that satire requires the audience to have a conceptual awareness of the existence of the author and their intention: the audience must understand the work to have a rhetorical purpose (and therefore understand that there is an author with an intent) in order to understand it as satirical at all. ${ }^{389}$

What matters more for my purposes than the praises of the critics, or the reproaches of detractors, is how players experience and understand the satire, rather than whether or not it succeeds in a literary sense (or whether it is particularly dignified). We might best understand this by returning to the idea that players experience directed freedom that arises from a dominance/submission relationship between the developer and the player. As in a BDSM scene, the author remains particularly important in dark or transgressive play (which I would argue includes the satire of GTA) as the orchestrator of an experience which the player is invited to work with. The player may experience the satire and authorial intent here not just as an interesting narrative element, but as a form of constraint or allowance on their play.

One interviewee in particular emphasized the importance of GTA's satire and social commentary: he recognized that his tendency to "always analyze and make connections" was connected to his interest in social and political commentary (and potentially even to his sense of self as a "politically and socially engaged" person). But importantly, the "compelling" and "fantastic" social commentary of the game, was a 
crucial aspect of why the game and the gameworld were absorbing and interesting to him:

AARON: [The constant joking] is also part of the environment that I find really immersive. Like, when you see the political ads, or when you see the billboards [...] or when you hear Rebel Radio, and things like that. It's my favourite because it's just like this parody of the worst of the US talk radio establishment"

This helps to illustrate the important relationship between immersion, realism, and satire in a digital world. Unlike conventional satire, the GTA 5 game world presents social commentary in an immersive way, allowing players to experience it rather than to read or watch it. The connection between humour and realism is an important theme for my interviewees. Here, Aaron explains that the parodic nature of the game creates a bridge between the game world and the real world and makes the game more realistic through its humorously hyperbolic treatment of real world things like radio and billboards:

AARON: I think humour is a way of making the game more accessible and more realistic. It links together [the game world and] the real world. And on one hand, your brain understands [it's] a parody, but on the other it draws you in in a different way because of that, and creates a more complete game world.

Interviewee two also discussed the idea that the game "was not serious" because of its satirical connection to the real world:

CONNOR: GTA is like a mime... like a mirror, but a more direct mirror [than other games]. It's like this because everything looks the same, everything sounds the same, people are speaking English for example, people look like people, the cities in GTA actually resemble a real city [...] And, they try to have a world that looks exactly like the real world, except everything that happens within it is exactly not how the real world works. [...] And even though GTA in terms of appearance looks quite a bit similar, everything in it is so, like, zany, wild and ... unpredictable is the wrong word... I guess predictable would be a better way of putting it because it doesn't matter what happens, it literally doesn't matter what happens in GTA 5. 
Scholars who are attempting to push back against the claims of media-effects research, often assert that the problematic in-game content is divorced from reality. For example, in his analysis of GTA 3 being banned in Australia, Finn argues that the satirical nature of the GTA series serves to undermine the gameworld's realism. He argues that self-referential satire (such as radio ads that mock gaming in GTA 3, or the character of Jimmy in GTA 5) works as anti-realism, that "reminds the player they are dealing with a fictional universe". ${ }^{390}$ Finn is aiming to distance the gameworld from the real world to discredit claims by media-effects researchers that videogames lead to real-world violence. However, for my participants, the characteristics of satire linked San Andreas explicitly to the real world. Here, the satirical depends fundamentally on linkages to the real world, and my participants either recognized the ways in which GTA 5 mirrored the real world, but made it hyperbolic, or the way in which satire made the in-game world feel more real.

For Aaron, satire and social commentary was a way of mediating between the real world and the game world. Satire was linked with immersiveness and world building, which in turn allowed GTA 5 to create an environment that was as realistic as possible without it being so realistic that it threatened the play and the associated pleasure. But satire was also connected to seriousness:

AARON: Those deeper more immersive moments [referring to moments of "really fantastic social commentary"] are what drew me in and made me feel legitimate. You almost have to legitimate liking it to yourself... because you can't admit that you like going around stealing cars and shooting people [...] So it's almost more legitimate to like the social commentary aspects, but I also do actually find it really funny and interesting.

390 Finn, "Political Interface: The Banning of GTA3 in Australia." 
While both the teller and the audience of humour and satire are implicated in the success of a joke, a conventional audience does not participate in the creation of the satire. ${ }^{391}$ The video game medium challenges this. As I have argued throughout the preceding chapters, the role of the player matters in how we interpret and analyze video games, although this role does not supersede or erase the importance of the game's developers. Rather, understanding this relationship through a kink-informed lens where power and control are negotiated, and are a powerful source of pleasure, is key. Goodrich argues that satire plays on the "ambiguities of words and the uncertainties of interpretation" and recreates or re-enacts real life in order to make it more "sensual and humourous". ${ }^{392}$ Both dark play and video games' natural affinity for the uncanny strengthen the potential for this to happen. The dissonance, or liminality, that exists between the ludological and narrative elements of the game, as well as between the player and avatar, make it a profoundly contingent medium.

\section{Race Humour}

Given the tremendous amount of satire focused on race in GTA 5, and the way that satire tends to destabilize meaning by making the lines between real and parody, and humour and serious blurry, it's important to reconsider the depiction of race (and especially race humour) in the game. One simple example is the title of this chapter, taken from one of the game's missions. Hood Safari has you attend a drug-deal that goes sideways, and you

\footnotetext{
391 Lockyer and Pickering, "You Must Be Joking: The Sociological Critique of Humour and Comic Media," 3.

392 Goodrich, "The Importance of Being Earnest," 51.
} 
are required to shoot your way out of Grove Street (the central neighbourhood in the GTA: San Andreas) as waves of gang members arrive. I found the mission's content to be squeamishly problematic and stereotypical, but it's also absurd and amusing. Like many missions with this sort of affective blend, I found this mission to be particularly memorable. The title hints at the mission's awareness that players are looking for "a fix of inner-city realism" in much the same way real-life ghetto tourism provides. ${ }^{393}$ Given the game is targeting a presume white-male audience, the title suggests that the mission is poking fun as much at white fetishization of poverty and violence as it is commenting on actual life in urban ghettos. This sort of satirical self-awareness and race-humour is characteristic of GTA more generally, something that should not be overlooked in an examination of how racism and stereotypes are mobilized in the game.

Writing about the silver-age all-black porn market, Nash writes that "these films' explicitly racialized titles, often absurd plots, and comical narratives allowed the spectator to laugh at the all-black cast while also taking pleasure in the black bodies that populated the pornographic screen". ${ }^{394}$ Race humour is certainly a central part of GTA 5, and it is worth considering the ways in which Nash's argument may apply to the game.

Nash aims to examine the humourous work that race performs in pornography, namely the connection between hyperbolic absurdity and racial eroticism. ${ }^{395}$ She concludes that race-humour can help show how "painful racial fictions are often played with, exaggerated, rendered absurd, deflated, and even rendered exciting and sexy" ${ }^{396}$

393 Turton, “'Ghetto Tourism': New York's Disturbing New Trend."

${ }^{394}$ Nash, The Black Body in Ecstasy, 126.

395 Nash, 127.

396 Nash, 127. 
This is reminiscent of player's experiences of the GTA 5 gameworld and its depiction of race. However, this approach to humour is also a "representational and political tightrope" with as much potential to reinforce negative stereotypes as poke fun at them. ${ }^{397}$ Importantly, humour allows us to name the fantasies about race that uphold racial pleasure through their unspoken erasure. ${ }^{398}$ Because race pleasure is reliant on the erasure of the pleasure/humiliation that characterizes the relationship between white/black, race humor is therefore a threat to the stability of the colourline because it draws attention to this dynamic, exposes it and makes it absurd or laughable. ${ }^{399}$

For example, Nash argues that race humour in the film Black Taboo is present in the ways the film shows the connection between blackness and taboo, or the ways in which "black bodies fail to comply with the demands of the normative" ${ }^{400}$ It does so namely through the juxtaposition of religion and family values with incest. In the film, the actress who plays the family's mother, Veranda, discovers that the man who she thought was her son, and with whom she is having sex, is in fact not her son. She dismisses the absurd misunderstanding, saying "Well, you know what they say: we all look alike!". 401 Drawing on Miller Young, Nash argues that this simultaneously entrenches racial fictions while also serving as a "condemnation of the spectator's viewing pleasures", hinting towards the viewer's desire to buy into racial fictions (in this case, the idea that all black bodies are inseparable and serve the same sexualized function within the film) to

\footnotetext{
397 Nash, 90.

398 Nash, 110, 127.

399 Farley, "The Black Body as Fetish Object"; Nash, The Black Body in Ecstasy.

400 Nash, The Black Body in Ecstasy, 117.

401 Nash, 119.
} 
facilitate their pleasure. ${ }^{402}$ The pleasure of racial humour, she explains is that it "flirts with collectively held racial fictions without ever explicitly engaging questions of race", making race both "ubiquitous and absent". 403

GTA 5 is riddled with dialogue that has the same effect on the player. For example, Franklin's eternally bumbling friend Lamar pushes back against Franklin's hesitance to remain engaged in gang activity, saying: "gangs is positive. They all we got, my n---. That's our heritage". The humour in this statement comes from the player's understanding of the racial stereotype regarding the role of gangs in black neighbourhoods, and the apparently innately violent nature of these communities. Further, the statement draws attention to the what bell hooks calls gangsta culture, the capitalist and patriarchal hustle in which drugs and gangs become a "vibrant but deadly" economy, accepted into black life because of the potential to make a lot of money quickly. ${ }^{404}$ As hooks says, this fantasy of easy money is disseminated through media. Therefore, this line from Lamar serves both as an acknowledgement of this trope and the way GTA has been accused of perpetuating it. My experience with this sort of humour in GTA is that it is both pleasurable in that players feel like they "get" the social commentary, or are in on the joke, but that it is also deeply uncomfortable. These tensions and the enjoyment that results not only from the humour but also the push and pull of pleasure and anxiety itself. Furthermore, these moments gave me the sensation of being watched or observed by the game or the developers, something I think is best

402 Miller-Young, A Taste for Brown Sugar; Nash, The Black Body in Ecstasy.

403 Nash, The Black Body in Ecstasy, 121.

404 hooks, We Real Cool, 19. 
explained as a moment where the dominant/submissive relationship between developer and player is most visible. This feeling illuminates how the developer sets up a scenario for the player to react to, and that the player is also aware of the influence of the developer in these scenes and recognizes them as an actor with agency who is "present" in the game.

Lamar's statement also pushes back against Franklin's own narrative. Franklin is a "ghetto protagonist", a character whose ghetto identity is liminal and temporary, as he transforms into the "bourgeois protagonist" with expensive cars, and a lavish but cold house in the Hollywood hills. ${ }^{405} \mathrm{He}$ is "situationally ghetto", with a "streetwise and hip" appearance and who is able to operate with violence, but only when provoked. ${ }^{406}$ Lamar on the other hand, is Franklin's foil, a ghetto antagonist, characterized by his inability to use the resources available to him to become upwardly mobile. He is portrayed as being uneducated, hypersexual, and criminal, and often as a "common street thug, gangster, pimp, or other criminal" ${ }^{407}$ The interplay between the two characters can be experienced as a sort of negotiation between representations of blackness. While Franklin is arguably a more "positive" depiction than Lamar is, Lamar serves as a reminder that our interest in Franklin is emblematic of the desire of white audiences to buy into racial fictions about redemption. Lamar rejects redemption, claiming that gangs are "our heritage". Like Veranda's comment that "we all look alike", this comment both reproduces racist stereotypes while simultaneously pointing towards

\footnotetext{
405 Taylor and Johnson, "'Class, Meet Race': A Critical Re-Scripting of the Black Body through Ghetto and Bourgeois Characters in American Films," 119.

406 Taylor and Johnson, 120

407 Taylor and Johnson, 119.
} 
the racially-charged pleasure the viewer takes in playing through these stereotypical situations involving gang violence or ghetto protagonists.

In another instance, Lamar confronts Jimmy, Michael's son:

LAMAR: Who you callin a $n---$ ?

JIMMY: No, no. I'm not callin' nobody a n---

LAMAR: Man, what the fuck?

JIMMY: I...I mean... n-word. I....... that's not cool. Man, I don't say that.

For his part, Jimmy is a commentary on the presumed player; chubby, slobbish, and pale, he spends the bulk of his time locked in his room playing video games. If the player, playing as Franklin, chooses to hang out with Jimmy during gameplay, Jimmy will engage in cringe-worthy dialogue ("the homies is riding again!", "yeah boy l'm gangster now", "holla at you, fam"). During one of these hangout sessions, Jimmy will ask Franklin if he's angry because of his life circumstances, and will then proclaim

JIMMY: 'cause I'm angry, you know, I kinda wish I'd been born black. 'cause that'd explain my anger

FRANKLIN: Dog, that don't make any sense at all. Black folks don't always have to be angry.

Later, when Jimmy asks Franklin if he is a member of the Grove Street gang, Franklin explains that he is not:

FRANKLIN: So, no, you ain't living out some suburban gangbanger fantasy shit with me

Importantly, the interactions that lead to these conversations are optional and require the player to make the decision to repeatedly engage with Jimmy. Both of these pieces of dialogue are meant to be humourous, but their comedy depend on the player's ability to recognize not only racial stereotypes, but the pleasure that white players take in playing the game as Franklin. In these moments the player is able to share in and identify with 
Franklin's rebuke of Jimmy's attempts to claim "black coolness" through association, although this simultaneously depends on their acknowledgement of their own similarity to Jimmy.

Tellingly, after Jimmy and Franklin emerge from the bar, Jimmy makes increasingly explicit sexual advances on Franklin. While this seems to be played for homophobic laughs, the humour again depends on the player's acknowledgement of the ways in which black men's sexuality is both coveted and feared by white men. ${ }^{408}$ These scenes therefore use humour in a way that draws into question the player's own desires and their own motivations for playing Franklin and make the player's racial desires and pleasures the real punchline. ${ }^{409}$

While Phiddian cautions us against reading satire in too celebratory a way, denying the importance or power of satire is not an adequate corrective. While it is important not to unquestioningly applaud whatever message comes wrapped in satire, it is also insufficient to "avert our eyes and write such work off as unfunny". ${ }^{410}$ It may be particularly important to pay attention to satirical content that has "unpleasant political and ideological views", arguably including GTA. ${ }^{411}$ Instead, as Phiddian suggests, the solution may be to examine the range of responses that the satire evokes in players and to examine the actual effects of satire- which is often "limited or hit-and-miss, often counterproductive, seldom directly successful, sometimes little more than a consolation

\footnotetext{
408 hooks, We Real Cool, 67.

409 Nash, The Black Body in Ecstasy, 125.

410 Phiddian, "Satire and the Limits of Literary Theories," 54.

411 Phiddian, 54.
} 
for the defeated or merely grumpy". ${ }^{412}$ The question of whether or not the satire in GTA 5 is successful is less important than whether or not players experience it as such. As Phiddian explains, "the satirical is not brute, formal facts about texts", but rather depends on our ability to perceive rhetorical purpose through gameplay. ${ }^{413}$

\section{Chapter 6 conclusions}

In this chapter, I have aimed to show that it is worthwhile to take a second look at the depiction of race in GTA 5, in a way that attempts to avoid knee-jerk or essentializing readings. Certainly, the game presents problematic stereotypes and representations of blackness, that may reinforce racist ideas in certain players. However, some of the work on race in GTA follows closely in the analytic footsteps of radical feminist analysis of pornography. Their unilateral focus on societal harm foregrounds cause-and-effect interpretations of the game, which I argue is overly simplistic.

Instead, I have foregrounded the way dark play helps to destabilize the assumed meanings and introduce nuance to GTA's treatment of race. I have focused on how GTA's race-play is a form of dark play where real racial stereotypes and biases are brought to the table and twisted, subverted, or hyperbolized in a way that more closely resembles kinky race-play than $19^{\text {th }}$ century minstrelsy. Secondly, I have argued that satire and race humour accomplish a similar manoeuvre. While these may still be problematic depictions of race, they do hinge on an acknowledgement of racial ideology and stereotypes. By

\footnotetext{
412 Phiddian, 53.

413 Phiddian, 46.
} 
making these visible, race-play and race humour also have the potential to render them absurd, and possibly less powerful. 


\section{Conclusion: BUSTED! Re-reading GTA 5 for pleasure and anxiety}

This project grew out of an interest in thinking critically about pornography and pornography literacy. I first attended an anti-pornography workshop put on by an agency that worked closely with the grade school system during my time working in sexual health in Nova Scotia. These assaultive presentations often show audiences countless decontextualized images in an effort to shock them into believing what anti-pornography feminists believe: that pornography is harmful and that signifiers (the money shot, a woman being choked) always signify tangible harm being done against real women. Despite occurring far after the height of the Sex Wars, the issue of pornography can still provoke deep divisions, hurt, and anger within feminist movements, something I caught a small glimpse of in the weeks after the workshop.

Years later, watching Sarkeesian's Tropes vs Women in Video Games series, I was struck by how similar the affective experience of watching scene after scene of decontextualized virtual violence was to the anti-pornography presentations. It is also clear that many of the same strong emotions are attached to this issue as to pornography. However, with games as with pornography, I am convinced that these approaches are ineffective for teaching media literacy regardless of one's personal feelings about pornography and the sex acts that were being treated as stand-ins for violence, or video games and the interactive violence they often contain. While the practicalities of media literacy are beyond the scope of this project, I hope that my 
contribution can help challenge essentializing radical feminist approaches to videogames and provoke us to find ways of engaging with transgressive texts with increasing nuance.

The purpose of this project has been to explore how the lessons learned about feminist media analysis in the sex wars and the subsequent debates about violent or transgressive pornography can help us make sense of and develop more nuanced understandings of transgressive video games. Radical feminist approaches to pornography tended to focus on the potential for harm and used media-effects research to support these claims. Ultimately, they were able to mobilize these arguments in service of legal interventions and shifting public discourse. Despite advances in the pornography debates over the past three decades, I have argued that these same issues are playing out in a new arena: anti-violence in video games advocacy. Whereas many young feminists have developed nuanced analyses of pornography, I felt that much of the public discourse about games tended to essentialize these games and rely on old standby arguments that would not have been considered sufficient in a sophisticated discussion of pornography. Rather than rehash these, I have attempted to contribute to the rapidly growing field of research that eschews knee-jerk condemnation of games and gameplay that might, on their face, be considered problematic.

We are living in a world increasingly saturated with videogames, which are likely to become more realistic and immersive as technology and our ability to fully mobilize the medium grow and advance. In this landscape, understanding games (and, more importantly, teaching young people to think and engage critically with them) will require us to depart from radical feminist approaches to authoritative textual readings. It is 
imperative that we are able to talk about and teach games in a way that considers their contingencies, subjectivities, and uncomfortable pleasures.

Using autoethnography, interpretative phenomenological analysis and textual analysis, I have attempted to re-read Grand Theft Auto 5 in a way that acknowledges the complicated and contingent pleasures of transgression. In doing so I have relied on the concept of dark play, and a kink-informed theoretical lens to elucidate the complex and ever-shifting relationship between transgression, pleasure and anxiety. Focussing on transgression- the way players break or subvert norms- and how this is often an erotic, and liminal experience that tends to result in a fracturing of the self has helped me to demonstrate that cause-and-effect analyses of violence in games are insufficient. My intent is not to dismiss the concerns of critics who point out the violent, racist and sexist content of these games. Rather, I aim to show that it is difficult to understand the meaning of dark play without considering the numerous ways players negotiate this meaning and hold seemingly disparate interpretations in tension with one another.

One critical aspect of this has been a kink-centric analysis of the relationship between player and developer. While dark play allows players to subvert or transgress norms, a kink analysis helps to elucidate the role of the developer and their relationship to the player. I have argued that the dominant/submissive relationship between developer and player augments the affective potential of dark play and helps to challenge feminist readings of violent games that argue they are about uncomplicated male power fantasies or sadistic voyeurism. 
In particular, I have explored how GTA 5's torture scenes can be re-read as uncanny instances of psychosexual pleasure. I have argued that the developer takes the role of dominant here and helps to bring to the surface transgressive desires ordinarily hidden from the conscious of the submissive player. The player therefore experiences a fracturing or dissolution of self where they simultaneously experience the role of violator/violated, dominant/submissive. This presents a challenge to radical feminist readings that focus on sadistic voyeurism.

In the final section of my project I have attempted to re-read the racial stereotypes in GTA 5 as examples of race-play. I argue that as a kinky sexual practice, race play does depend on actual racism and stereotypes, but has the potential to subvert or challenge these because it depends on a conscious acknowledgement of their erotic power. This is in contrast to racial pleasure, which Farley argues upholds the colourline, and depends on the erasure of the sadomasochistic nature of racial ideology. Similarly, I've argued that satire and race humour serve an analogous function: they depend on explicit acknowledgement of racial hegemony and by recognizing it, they have the potential to make it absurd. Both race-play and race humour are forms of dark play wherein the player derives pleasure from the way their identity is held in tension between humourous/ serious, authentic/ stereotyped, and anxiety/ pleasure. Of course, this does not forgive GTA or any other game for its racism but instead suggests that understanding the impact of stereotypes and bias in video games requires a more nuanced approach than media effects and radical feminist media analysis offers. 
I hope that this research has made a small contribution towards bringing the fields of pornography studies and games studies closer together. While game developers and scholars have clearly shown the benefit of using a kink lens in games analysis, I believe that an even closer academic kinship would be beneficial. There is inherent value in adapting analytical approaches used for pornography to video games. The connection between the two goes beyond media theory, as Aaron explains:

AARON: People think about [games] sort of like porn... like, "I don't do it, I don't really approve of it, but they're going to do it because it's out there" [...] I've never dated anyone who was an active gamer so I find they tend to be... well you know your boyfriend watches porn, you know your boyfriend plays games, it's sort of a necessary evil [...] There's also that stigma attached to games still I think [...] so I find when you're dating new people it's something, once again like porn in that you're kind of scared to bring it up and have that conversation. I would never talk about video games on the first date, you know what I mean? [laughs]

Studying games and pornographic texts side by side often helps to elucidate the complex nature of our affective responses to transgressive experiences. Importantly, the debates that have occurred over decades about pornography, violence and harm provide an abundance of points of departure for games scholars interested in similar questions. It would be unfortunate to overlook the opportunity to draw these two disciplines into closer conversation with further work and research.

Understanding and taking seriously games as spaces where players can explore non-normative pleasures like rape fantasy and race play is inherently valuable, even when it is uncomfortable. ${ }^{414}$ Ultimately my hope is that my small contribution to games studies will link it more explicitly to important histories like the feminist debates about

414 Khan, "Fetishizing Music as Rape Culture," 28. 
pornography. As Clover argues, the standard critiques of violent and transgressive games needs "not only a critical but a political interrogation". ${ }^{415}$ I believe that doing so can only make the discipline richer, and at best can help avoid the ahistorical recreation of simplistic binaries.

This project has been an effort to explore my own experience of a complex and transgressive text. I believe that there are exciting possibilities in terms of future work on the phenomenology of games, and how games are experienced as multi-sensory texts that are co-constituted between player and machine. While this project has focused on applying the theory and methods of pornography studies to a violent and transgressive game, increasingly games are becoming explicitly pornographic. This combination of the virtual with sex and sexuality will require a robust interdisciplinary study. I believe this project provides a solid foundation from which to begin exploring more challenging and contentious texts.

${ }^{415}$ Clover, Men, Women, and Chain Saws, 19. 


\section{Bibliography}

Aarseth, Espen J. Cybertext: Perspectives on Ergodic Literature. Baltimore, Md: Johns Hopkins University Press, 1997.

Adachi, Paul J.C., and Teena Willoughby. "The Effect of Violent Video Games on Aggression: Is It More than Just the Violence?" Aggression and Violent Behavior 16, no. 1 (January 2011): 55-62. https://doi.org/10.1016/j.avb.2010.12.002.

Albury, Kath. "Reading Porn Reparatively." Sexualities 12, no. 5 (October 2009): 647-53. https://doi.org/10.1177/1363460709340373.

Allen, Mike, Dave D'Alessio, and Keri Brezgel. "A Meta-Analysis Summarizing the Effects of Pornography II Aggression After Exposure." Human Communication Research 22, no. 2 (December 1995): 258-83. https://doi.org/10.1111/j.14682958.1995.tb00368.x.

Amanda Wallace. "Is GTA V's Graphic Torture Scene a Step Too Far," September 20, 2013. https://www.gameskinny.com/ai1r2/is-gta-vs-graphic-torture-scene-a-steptoo-far.

Anderson, C. A., and B. J. Bushman. "Effects of Violent Video Games on Aggressive Behavior, Aggressive Cognition, Aggressive Affect, Physiological Arousal, and Prosocial Behavior: A Meta-Analytic Review of the Scientific Literature."

Psychological Science 12, no. 5 (September 1, 2001): 353-59. https://doi.org/10.1111/1467-9280.00366.

"Are Video Games the Key to World Peace?" CBC News. Accessed April 5, 2017. http://www.cbc.ca/news/entertainment/empathy-video-games-unesco-study1.3985702 .

Babcock, Barbara A., and American Anthropological Association, eds. The Reversible World: Symbolic Inversion in Art and Society: [Papers]. Symbol, Myth, and Ritual Series. Ithaca: Cornell University Press, 1978.

Barker, Meg. "Psychology and Pornography: Some Reflections." Porn Studies 1, no. 1-2 (January 2, 2014): 120-26. https://doi.org/10.1080/23268743.2013.859468.

Bass, Alan. "The Double Game: An Introduction." In Taking Chances: Derrida, Psychoanalysis, and Literature, edited by Joseph $\mathrm{H}$ Smith and William Kerrigan, 66-84. JHU Press, 1988.

Bataille, Georges, Mary Dalwood, and Colin McCabe. Eroticism, 2012.

Bernstein, Joseph. "The Franklin Conspiracy: Why Gamers Decided The Police In 'GTA V' Are Racist." BuzzFeed. Accessed March 11, 2018. https://www.buzzfeed.com/josephbernstein/the-franklin-conspiracy-whygamers-decided-the-police-in-gt.

Blow, Jonathan. "Fundamental Conflicts in Contemporary Game Design." presented at the Montreal International Game Summit., Montreal, Quebec, 2008. http://braidgame.com/news/2008/11/a-new-lecture-about-story-and-game-design/.

Bogost, lan. How to Talk about Videogames. Minneapolis,: University of Minnesota Press, 2015. 
http://search.ebscohost.com/login.aspx?direct=true\&scope=site\&db=nlebk\&db= nlabk\&AN=991812.

Brice, Mattie. "Play and Be Real About It: What Games Could Learn From Kink." In Queer Games Studies, edited by Bonnie Ruberg and Adrienne Shaw. Minneapolis, MN: University of Minnesota Press, 2017.

Brown, Ashley ML. "Three Defences for the Fourteen-Inch Barbed Penis: Darkly Playing with Morals, Ethics, and Sexual Violence." In Dark Side of Game Play., edited by Jonas Linderoth, Torill Elvira Mortensen, and Ashley ML Brown. Hoboken, United States: Taylor and Francis, 2015.

Brown, Emily, and Paul Cairns. "A Grounded Investigation of Game Immersion." CHI'O4 Extended Abstracts on Human Factors in Computing Systems, no. 1297-1300 (2004).

Brown v. Entertainment Merchants Association, 564 U.S. 786 (2011).

Byrd, Patrick R. "It's All Fun and Games Until Someone Gets Hurt: The Effectiveness of Proposed Video-Game Legislation on Reducing Violence in Children." Houston Law Review 22 (2007): 401.

Cerbone, David R. Understanding Phenomenology. Repr. Understanding Movements in Modern Thought. Stocksfield: Acumen, 2010.

Chávez, Minerva S. "Autoethnography, a Chicana's Methodological Research Tool: The Role of Storytelling for Those Who Have No Choice but to Do Critical Race Theory." Equity \& Excellence in Education 45, no. 2 (April 2012): 334-48. https://doi.org/10.1080/10665684.2012.669196.

- - - "Autoethnography, a Chicana's Methodological Research Tool: The Role of Storytelling for Those Who Have No Choice but to Do Critical Race Theory." Equity \& Excellence in Education 45, no. 2 (April 2012): 334-48. https://doi.org/10.1080/10665684.2012.669196.

Cixous, Hélène. Three Steps on the Ladder of Writing. New York: Columbia University Press, 1993.

Clover, Carol J. Men, Women, and Chain Saws: Gender in the Modern Horror Film. First Princeton classics edition. Princeton Classics. Princeton, New Jersey: Princeton University Press, 2015.

Cohen, Jonathan. "Defining Identification: A Theoretical Look at the Identification of Audiences With Media Characters." Mass Communication and Society 4, no. 3 (August 2001): 245-64. https://doi.org/10.1207/S15327825MCS0403_01.

Condren, Conal. "Satire and Definition." Humor 25, no. 4 (January 14, 2012). https://doi.org/10.1515/humor-2012-0019.

Cossman, Brenda. "Cossman, Brenda. 'Disciplining the Unruly: Sexual Outlaws, Little Sisters and the Legacy of Butler.' U. Brit. Colum. L. Rev. 36 (2003): 77. Harvard." University of British Columbia Law Review 36 (2003): 77-99.

Crane-Seeber, Jesse Paul. "Sexy Warriors: The Politics and Pleasures of Submission to the State." Critical Military Studies 2, no. 1-2 (May 3, 2016): 41-55. https://doi.org/10.1080/23337486.2016.1144402. 
"Damsel in Distress (Part 2) Tropes vs Women." Feminist Frequency (blog), May 28, 2013. https://feministfrequency.com/video/damsel-in-distress-part-2-tropes-vswomen/.

Daniel, D. "'Why Be Something That You're Not?': Punk Performance and the Epistemology of Queer Minstrelsy." Social Text 31, no. 3116 (September 1, 2013): 13-34. https://doi.org/10.1215/01642472-2152819.

Day, Terri, and Ryan C.W. Hall. "Déjà Vu: From Comic Books to Video Games: Legislative Reliance on'Soft Science'to Protect Against Uncertain Societal Harm Linked to Violence v. The First Amendment." Oregon Law Review 89, no. 2 (2010): 415-52.

Delamere, Fern M., and Susan M. Shaw. "Playing with Violence: Gamers' Social Construction of Violent Video Game Play as Tolerable Deviance." Leisure/Loisir 30, no. 1 (January 2006): 7-25. https://doi.org/10.1080/14927713.2006.9651339.

Denzin, Norman. "The Many Faces of Emotionality: Reading Persona." In Investigating Subjectivity: Research on Lived Experience, edited by Carolyn Ellis and Michael Flaherty, 17-30, 1992.

Derrida, Jacques, and Francois Ewald. "A Certain 'Madness' Must Watch over Thinking." Educational Theory 45, no. 3 (September 1995): 273-91. https://doi.org/10.1111/j.1741-5446.1995.00273.x.

Dill, Karen E., and Kathryn P. Thill. "Video Game Characters and the Socialization of Gender Roles: Young People's Perceptions Mirror Sexist Media Depictions." Sex Roles 57, no. 11-12 (December 3, 2007): 851-64. https://doi.org/10.1007/s11199-007-9278-1.

Dill, Karen, Douglas A Gentile, William A Richter, and Jody C Dill. "Violence, Sex, Race and Age in Popular Video Games: A Content Analysis." Featuring Females: Feminist Analyses of the Media, 2005, 115-30.

Dworkin, Andrea. Pornography: Men Possessing Women. London: Women's Press, 1981.

Dymock, Alex. "But Femsub Is Broken Too! On the Normalisation of BDSM and the Problem of Pleasure." Psychology and Sexuality 3, no. 1 (January 2012): 54-68. https://doi.org/10.1080/19419899.2011.627696.

Eastin, Matthew S. "Video Game Violence and the Female Game Player: Self- and Opponent Gender Effects on Presence and Aggressive Thoughts." Human Communication Research 32, no. 3 (July 2006): 351-72. https://doi.org/10.1111/j.1468-2958.2006.00279.x.

Ellis, Carolyn. The Ethnographic I: A Methodological Novel about Autoethnography. Ethnographic Alternatives Book Series, v. 13. Walnut Creek, CA: AltaMira Press, 2004.

Engelhardt, Christopher R., Bruce D. Bartholow, Geoffrey T. Kerr, and Brad J. Bushman. "This Is Your Brain on Violent Video Games: Neural Desensitization to Violence Predicts Increased Aggression Following Violent Video Game Exposure." Journal of Experimental Social Psychology 47, no. 5 (September 2011): 1033-36. https://doi.org/10.1016/j.jesp.2011.03.027.

Equality Now. "Japan: Rape Simulator Games and the Normalization of Sexual Violence," 2011. 
https://web.archive.org/web/20110702031507/http://www.equalitynow.org/tak e_action/japan_action332.

Ermi, Laura, and Frans Mäyrä. "Fundamental Components of the Gameplay Experience:

Analysing Immersion." Worlds in Play: International Perspectives on Digital Games Research 37, no. 2 (2005): 37-53.

Everett, Anna, and Craig Watkins. "The Power of Play: The Portrayal and Performance of Race in Video Games." In The Ecology of Games: Connecting Youth, Games, and Learning, edited by Kim Salen, 141-66. The John D. and Catherine T. MacArthur Foundation Series on Digital Media and Learning. Cambridge, MA: The MIT Press, n.d.

Farley, Anthony Paul. "The Black Body as Fetish Object." Oregon Law Review 76 (1997): 457-535.

Ferguson, Christopher J. "Evidence for Publication Bias in Video Game Violence Effects Literature: A Meta-Analytic Review." Aggression and Violent Behavior 12, no. 4 (July 2007): 470-82. https://doi.org/10.1016/j.avb.2007.01.001.

Ferguson, Christopher J., and John Kilburn. "The Public Health Risks of Media Violence: A Meta-Analytic Review." The Journal of Pediatrics 154, no. 5 (May 2009): 759-63. https://doi.org/10.1016/j.jpeds.2008.11.033.

Ferguson, Christopher J., and Stephanie M. Rueda. "The Hitman Study: Violent Video Game Exposure Effects on Aggressive Behavior, Hostile Feelings, and Depression." European Psychologist 15, no. 2 (January 2010): 99-108.

https://doi.org/10.1027/1016-9040/a000010.

Finn, Mark. "Political Interface: The Banning of GTA3 in Australia." In The Meaning and Culture of Grand Theft Auto: Critical Essays, edited by Nate Garrelts, 35-48. Jefferson, NC: McFarland \& Co, 2006.

Foucault, Michel. The History of Sexuality: An Introduction, Volume I. New York, N.Y.: Vintage, 1990.

Fox Harrell, D., and Jichen Zhu. "Agency Play: Dimensions of Agency for Interactive Narrative Design." AAAI Spring Symposium: Intelligent Narrative Technologies II., 2009.

Frasca, Gonzalo. "Ludologists Love Stories, Too: Notes from a Debate That Never Took Place.," 2003.

Freud, Sigmund. "The 'Uncanny.'" In The Standard Edition of the Complete Psychological Works of Sigmund Freud, Volume XVII (1917-1919): An Infantile Neurosis and Other Works. 217-256, 1919.

FutureMajority. Hillary Clinton Hates On Video Games. Accessed February 18, 2017. https://www.youtube.com/watch?v=x1udjd2Aq3E\&feature=youtu.be.

Galbraith, Patrick W. "RapeLay and the Return of the Sex Wars in Japan." Porn Studies 4, no. 1 (January 2, 2017): 105-26. https://doi.org/10.1080/23268743.2016.1252159.

Gannon, Susanne. "The (Im)Possibilities of Writing the Self-Writing: French Poststructural Theory and Autoethnography." Cultural Studies <=> Critical Methodologies 6, no. 4 (November 1, 2006): 474-95. https://doi.org/10.1177/1532708605285734. 
Gerbner, George, Larry Gross, Michael Morgan, and Nancy Signorielli. "Living with Television: The Dynamics of the Cultivation Process." In Perspectives on Media Effects, 17-40, 1986.

Goodrich, Peter. "The Importance of Being Earnest: Satire and the Criticism of Law." Social Semiotics 15, no. 1 (April 2005): 43-58. https://doi.org/10.1080/10350330500059122.

Grimshaw, Mark. "Sound and Immersion in the First-Person Shooter." Games Computing and Creative Technologies: Journal Articles (Peer-Reviewed), 2008. http://digitalcommons.bolton.ac.uk/gcct_journalspr/3.

"GTA 5 Has Sold-In 85 Million Copies." IGN. Accessed December 11, 2017. http://www.ign.com/articles/2017/11/07/gta-5-has-sold-in-85-million-copies.

"GTA V - Stormfront." Accessed May 23, 2018. https://www.stormfront.org/forum/t963832/.

Hall, Ryan C.W., Terri Day, and Richard C.W. Hall. "A Plea for Caution: Violent Video Games, the Supreme Court, and the Role of Science." Mayo Clinic Proceedings 86, no. 4 (April 2011): 315-21. https://doi.org/10.4065/mcp.2010.0762.

Hall, Stuart. "What Is This 'Black'in Black Popular Culture?" edited by Stuart Hall, 479-89. Routeldge, 2006.

Haraway, Donna. "A Cyborg Manifesto: Science, Technology, and Socialist-Feminism in the Late Twentieth Century." In Simians, Cyborgs and Women, 149-81. New York, NY: Routledge, 1991.

Hernandez, Patricia. "Someone Actually Tried Testing Out If GTA V Cops Are 'Racist."' Kotaku. Accessed March 12, 2018. https://kotaku.com/someone-actually-triedtesting-out-if-gta-v-cops-are-ra-1691180811.

Hester, Helen. Beyond Explicit: Pornography and the Displacement of Sex. Albany: State University of New York Press, 2014.

Higgin, T. "Blackless Fantasy: The Disappearance of Race in Massively Multiplayer Online Role-Playing Games." Games and Culture 4, no. 1 (December 1, 2008): 3-26. https://doi.org/10.1177/1555412008325477.

hooks, bell. "Eating the Other: Desire and Resistance." In Black Looks: Race and Representation, 21-39. Boston: South End Press, 1992.

- - - We Real Cool: Black Men and Masculinity. New York: Routledge, 2004.

"How to Skip the Torture Scene? :: Grand Theft Auto V General Discussions." Accessed May 17, 2018. https://steamcommunity.com/app/271590/discussions/0/611704730317005301/

Jantzen, Gitte, and Jans F. Jensen. "Powerplay - Power, Violence and Gender in Video Games." Al \& Society 7, no. 4 (December 1993): 368-85. https://doi.org/10.1007/BF01891418.

Jennett, Charlene, Anna L Cox, Paul Cairns, Samira Dhoparee, Andrew Epps, Tim Tijs, and Alison Walton. "Measuring and Defining the Experience of Immersion in Games." International Journal of Human-Computer Studies 66, no. 9 (2008): 641-61.

Jones, Steve. "'Extreme' Porn? The Implications of a Label." Porn Studies 3, no. 3 (July 2, 2016): 295-307. https://doi.org/10.1080/23268743.2016.1196011. 
Keogh, Brendan. A Play of Bodies: How We Perceive Videogames. Cambridge, MA: MIT Press, 2018.

- - - "The Positive Discomfort of Spec Ops: The Line." Game Studies 16, no. 2 (2016).

- - - "Videogames Aren't Special. Videogames Aren't Unique." Brendan Keogh (blog), April 30, 2015. https://brkeogh.com/2015/04/30/videogames-arent-specialvideogames-arent-unique/.

Khan, Ummni. "Fetishizing Music as Rape Culture." Studies in Gender and Sexuality 18, no. 1 (January 2, 2017): 19-30. https://doi.org/10.1080/15240657.2017.1276782.

- - - "Hot For Kink, Bothered by The Law: BDSM and the Right to Autonomy." The Canadian Bar Association, Alberta Branch (blog), August 8, 2016. http://www.cbaalberta.org/Publications-Resources/Resources/Law-Matters/Law-MattersSummer-2016-Issue/Hot-for-Kink,-Bothered-by-the-Law-BDSM-and-the-Rig.

- - - Vicarious Kinks: S/m in the Socio-Legal Imaginary. Toronto ; Buffalo ; London: University of Toronto Press, 2014.

Kinsella, Elizabeth Anne. "Hermeneutics and Critical Hermeneutics: Exploring Possibilities within the Art of Interpretation." Forum Qualitative Sozialforschung/ Forum: Qualitative Social Research 7, no. 3 (2006).

Klevjar, Rune. "In Defense of Cutscenes," 2002.

Kuzmanovic, Dejan. "Queer Race Play: Kinky Sex and the Trauma of Racism." In Disgust and Desire: The Paradox of the Monster, edited by Kristen Wright, 71-88. Brill, 2018.

Lacity, Mary C, and Marius A. Janson. "Understanding Qualitative Data: A Framework of Text Analysis Methods." Journal of Management Information Systems 11, no. 2 (1994): 137-55.

Lacombe, Dany. Blue Politics Pornography and the Law in the Age of Feminism. Toronto: University of Toronto Press, 2016.

Leonard, David. "High Tech Blackface -- Race, Sports Video Games and Becoming the Other." Intelligent Agent 4, no. 4 (n.d.).

- - - "'Live in Your World, Play in Ours': Race, Video Games, and Consuming the Other." Studies in Media and Information Literacy 3, no. 4 (2003): 1-0.

- - - "Not a Hater, Just Keepin' It Real: The Importance of Race- and Gender-Based Game Studies." Games and Culture 1, no. 1 (January 2006): 83-88. https://doi.org/10.1177/1555412005281910.

- - - "Young, Black (\& Brown) and Don't Give a Fuck: Virtual Gangstas in the Era of State Violence." Cultural Studies $\leftrightarrow$ Critical Methodologies 9, no. 2 (April 2009): 248-72. https://doi.org/10.1177/1532708608325938.

Linderoth, Jonas, and Torill Elvira Mortensen. "Dark Play: The Aesthetics of Controversial Playfulness." In Dark Side of Game Play., edited by Jonas Linderoth, Torill Elvira Mortensen, and Ashley L Brown. Hoboken, United States: Taylor and Francis, 2015.

Lloyd-Smith, Allan Gardner. Uncanny American Fiction. London: Palgrave Macmillan UK, 1989. https://doi.org/10.1007/978-1-349-19754-5.

Lockyer, S., and M. Pickering. "You Must Be Joking: The Sociological Critique of Humour and Comic Media." Sociology Compass 2, no. 3 (n.d.): 808-20. 
MacKinnon, Catharine A. "Sexuality, Pornography, and Method: 'Pleasure under Patriarchy."” Ethics 99 (1989): 314-46.

Marshall, Ashley. "Gulliver, Gulliveriana, and the Problem of Swiftian Satire." Philological Quarterly 84, no. 2 (2002).

McCabe, Colin. "Introduction to the Penguin Edition." In Eroticism, by Georges Bataille, translated by Mary Dalwood, 2012.

McGonigal, Jane. Gaming Can Make a Better World. Accessed April 8, 2017. https://www.ted.com/talks/jane_mcgonigal_gaming_can_make_a_better_world.

McGonigal, Jane, and Inc OverDrive. Reality Is Broken. S.I.: Penguin Group US, 2011. http://api.overdrive.com/v1/collections/v1L2BaQAAAJcBAAA1M/products/44205f 90-d66a-4b2b-8483-8a0cdb6a8822.

Miller-Young, Mireille. A Taste for Brown Sugar: Black Women in Pornography. Durham ; London: Duke University Press, 2014.

Milne, Tom, Paul Willemen, and Phil Hardy. The Encyclopedia of Horror Movies. 1st U.S. ed. New York: Harper \& Row, 1986.

Monson, Melissa J. "Race-Based Fantasy Realm: Essentialism in the World of Warcraft." Games and Culture 7, no. 1 (January 2012): 48-71. https://doi.org/10.1177/1555412012440308.

Mori, Masahiro. "The Uncanny Valley [From the Field]." Translated by Karl MacDorman and Norri Kageki. IEEE Robotics \& Automation Magazine 19, no. 2 (June 2012): 98-100. https://doi.org/10.1109/MRA.2012.2192811.

Mortensen, Torill Elvira. "Keeping the Balance: Morals at the Dark Side." In Dark Side of Game Play., edited by Jonas Linderoth, Torill Elvira Mortensen, and Ashley L Brown. Hoboken, United States: Taylor and Francis, 2015.

Moustakas, Clark E. Phenomenological Research Methods. 6. print. Thousand Oaks, Calif.: Sage Publ, 1999.

Mulkay, Michael Joseph. On Humor: Its Nature and Its Place in Modern Society. Polity Press, 1988.

Mulvey, Laura. "Visual Pleasure and Narrative Cinema." Screen 16, no. 3 (1975): 6-18. Murray, Janet. "The Last Word on Ludology v Narratology in Game Studies." In . 2005.," 2005.

Murray, Janet Horowitz. Hamlet on the Holodeck: The Future of Narrative in Cyberspace. Cambridge, Mass: MIT Press, 1998.

Nash, Jennifer C. The Black Body in Ecstasy: Reading Race, Reading Pornography. Next Wave. Durham ; London: Duke University Press, 2014.

Navarro-Remesal, Víctor, and Shaila García-Catalán. "Let's Play Master and Servant: BDSM and Directed Freedom in Game Design." In Rated M for Mature: Sex and Sexuality in Video Games, edited by Matthew Wysocko and Evan W Lauteria, 11932. New York, NY: Bloomsbury Publishing Inc., 2015.

Newall, Michael. "An Aesthetics of Transgressive Pornography." In Aesthetics and Pornography, edited by Jerrod Levinson and Hans Maes, 206-28. Oxford University Press, 2012.

Nishi, Naomi W., Cheryl E. Matias, and Roberto Montoya. "Exposing the White Avatar: Projections, Justifications, and the Ever-Evolving American Racism." Social 
Identities 21, no. 5 (September 3, 2015): 459-73.

https://doi.org/10.1080/13504630.2015.1093470.

Phiddian, Robert. "Satire and the Limits of Literary Theories." Critical Quarterly 55, no. 3 (2013): 44-58.

Philaretou, Andreas G., and Katherine R. Allen. "Researching Sensitive Topics through Autoethnographic Means." The Journal of Men's Studies 14, no. 1 (2005): 65-78.

Phillips, Bonnie B. "Virtual Violence or Virtual Apprenticeship: Justification for the Recognition of a Violent Video Game Exception to the Scope of First Amendment Rights of Minors." Ind. L. Rev. 36 (2003): 1385." Indiana Law Review 36 (2003): 1385.

Pink, Sarah. Doing Visual Ethnography. 3rd edition. Los Angeles: SAGE, 2013.

Post, David G. "Post, David G. 'Sex, Lies, and Videogames: Brown v. Entertainment Merchants Association.' Cato Sup. Ct. Rev. (2010): 27." Cato Supreme Court Review, 2010, 27.

Poulet, Georges. "Phenomenology of Reading." New Literary History 1, no. 1 (October 1969): 53. https://doi.org/10.2307/468372.

Richards, Rose. "Writing the Othered Self: Autoethnography and the Problem of Objectification in Writing About IIIness and Disability." Qualitative Health Research 18, no. 12 (2008).

Runions, Kevin C. "Toward a Conceptual Model of Motive and Self-Control in CyberAggression: Rage, Revenge, Reward, and Recreation." Journal of Youth and Adolescence 42, no. 5 (May 2013): 751-71. https://doi.org/10.1007/s10964-0139936-2.

Sallis, John. "The Identities of the Things Themselves." In Philosophy and Science in Phenomenological Perspective, 183-94. Dordrecht: Springer, 1984.

Sapach, Sonja. "Let's Play with Research Methodologies: Autoethnographic Memory Work and Critical Theory Development Through a 'Let's Play' of TRAUMA." presented at the Canadian Games Studies Association Annual Conference, Toronto, Ontario, May 30, 2017.

Sarkeesian, Anita. Women as Background Decoration (Part 1). Tropes vs. Women. Feminist Frequency, 2014. https://feministfrequency.com/video/women-asbackground-decoration-tropes-vs-women/.

Schechner, Richard. "Playing." Play \& Culture 1, no. 1 (1988): 3-19.

Schott, Gareth, and Jasper van Vught. "Replacing Preconceived Accounts of Digital Games with Experience of Play: When Parents Went Native in GTA IV." In Transactions of the Digital Games Research Association, Vol. 1, 2011.

Sebag-Montefiore, Clarissa. "'Fed up with Fantasies for Male Teenagers': Fixing the Depiction of Women in Games." The Guardian, September 6, 2017, sec. Culture. http://www.theguardian.com/culture/2017/sep/06/fed-up-with-fantasies-formale-teenagers-fixing-the-depiction-of-women-in-games.

Sedgewick, Eve. Touching Feeling: Affect, Pedagogy, Performativity. Durham, NC: Duke University Press, 2003. 
Shapiro, Jordan. "How Video Games Can Save The World." Forbes. Accessed April 8, 2017. http://www.forbes.com/sites/jordanshapiro/2017/01/31/how-videogames-can-save-the-world/.

Shaw, Adrienne. "Rethinking Game Studies: A Case Study Approach to Video Game Play and Identification." Critical Studies in Media Communication 30, no. 5 (December 2013): 347-61. https://doi.org/10.1080/15295036.2012.701013.

Sicart, Miguel. The Ethics of Computer Games. Cambridge, Mass.: MIT Press, 2009.

Silver, Dan. "GTA 5 Review: Satire, Parody - and Gameplay to Show Rockstar Have Cracked Combat in Breathtaking Grand Theft Auto V." mirror, September 16, 2013. http://www.mirror.co.uk/news/technology-science/technology/gta-5review-satire-parody-2277607.

Smith, Brett, and Andrew C. Sparkes. "Narrative and Its Potential Contribution to Disability Studies." Disability \& Society 23, no. 1 (January 2008): 17-28. https://doi.org/10.1080/09687590701725542.

Smith, Jesus G., and Aurolyn Luykx. "Race Play in BDSM Porn: The Eroticization of Oppression." Porn Studies 4, no. 4 (October 2, 2017): 433-46. https://doi.org/10.1080/23268743.2016.1252158.

Smith, Jonathan A., Paul Flowers, and Michael Larkin. Interpretative Phenomenological Analysis: Theory, Method and Research. Los Angeles: SAGE, 2009.

Spry, Tami. "Performing Autoethnography: An Embodied Methodological Praxis." Qualitative Inquiry 7, no. 6 (December 2001): 706-32. https://doi.org/10.1177/107780040100700605.

Stallybrass, Peter, and Allon White. The Politics and Poetics of Transgression. London: Methuen, 1986.

Stoller, Robert J. Observing the Erotic Imagination. Yale U.P., 1992.

"Stormfront - GTA IV." Accessed May 23, 2018. https://www.stormfront.org/forum/printthread.php?t=383133\&pp=100.

Strivers, Camilla. "Stivers, Camilla. 'Reflections on the Role of Personal Narrative in Social Science.' (1993): 408-425." Signs 18, no. 2 (1993): 408-25.

Stuart, Keith. "GTA 5 Review: A Dazzling but Monstrous Parody of Modern Life." the Guardian, September 16, 2013. http://www.theguardian.com/technology/2013/sep/16/gta-5-review-grand-theftauto-v.

Studlar, Gaylyn. "Masochism and the Perverse Pleasures of the Cinema." Quarterly Review of Film Studies 9, no. 4 (September 1984): 267-82. https://doi.org/10.1080/10509208409361219.

Sullivan, Rachel E. "Rap and Race: It's Got a Nice Beat, but What about the Message?" Journal of Black Studies 33, no. 5 (2003): 605-22.

Tassi, Paul. "Can We Forgive Hillary Clinton For Her Past War On Video Games?” Forbes. Accessed February 18, 2017. http://www.forbes.com/sites/insertcoin/2016/02/05/can-we-forgive-hillaryclinton-for-her-past-war-on-video-games/. 
Taylor, Laurie N. “From Stompin' Mushrooms to Bustin' Heads: Grand Theft Auto III as Paradigm Shift." In The Meaning and Culture of Grand Theft Auto, edited by Nate Garrelts. Jefferson, North Carolina: McFarland \& Company, Inc., 2006.

Taylor, T. L. Play between Worlds: Exploring Online Game Culture. Cambridge, Mass: MIT Press, 2006.

Taylor, Toniesha L., and Amber Johnson. "'Class, Meet Race': A Critical Re-Scripting of the Black Body through Ghetto and Bourgeois Characters in American Films." In Scripting the Black Masculine Body: Identity, Discourse, and Racial Politics in Popular Media, edited by Ronald L. Jackson and Mark C. Hopson, 113-28. SUNY Series, the Negotiation of Identity. Albany: State University of New York Press, 2006.

Tinwell, Angela, Nabi Abdel, and John P. Charlton. "Perception of Psychopathy and the Uncanny Valley in Virtual Characters." Computers in Human Behavior 29, no. 4 (2013): 1617-25.

Turton, Jonathan. "'Ghetto Tourism': New York's Disturbing New Trend." Dazed, June 2, 2017.

Valverde, Mariana. Sex, Power, and Pleasure. Women's Press Issues. Toronto, Ont: Women's Press, 1985.

Walter, Damian. "Let's Be Honest: White Male Geeks like Me Do Have a Problem." The Independent, April 7, 2016. http://www.independent.co.uk/voices/if-youre-amale-geek-who-acts-out-sexist-fantasies-with-muscular-characters-youre-nobetter-than-a6973086.html.

Young, Iris Marion. Throwing like a Girl and Other Essays in Feminist Philosophy and Social Theory. Bloomington: Indiana University Press, 1990.

Zita, Jacquelyn N. Body Talk: Philosophical Reflections on Sex and Gender. Between Men-between Women. New York: Columbia University Press, 1998.

\section{Cases}

Brown v. Entertainment Merchants Association, 564 U.S. 786 (2011).

Ginsberg v. New York, 390 U.S. 629 (1968)

Miller v California, 413 U.S. 15 (1973)

R. v. Butler, [1992] 1 S.C.R. 452

\section{Games and Films}

BioWare Austin. 2011. Star Wars: The Old Republic. Electronic Arts

DMA Designs. 1997. Grant Theft Auto. ASC Games

Id Software. 1993. DOOM. GT Interactive

IO Interactive. 2016. Hitman. Square Enix

Maxis. 2000. The Sims. Electronic Arts.

Powell, M, dir. 1960. Peeping Tom. United Kingdom.

Rockstar North. 2004. Grand Theft Auto: San Andreas. Rockstar Games 
Rockstar North. 2008. Grand Theft Auto IV. Rockstar Games

Rockstar North. 2013. Grand Theft Auto V. Rockstar Games

Wadd, Dick, dir. 2001. Niggas' Revenge. USA. 


\section{Appendix 1: Interview Script}

\section{Gameplay}

1. Could you tell me a brief history of your experience playing GTA? (When did you play, have you played previous titles, where do you play, alone or with others?).

a) Could you describe what a typical play session looks like?

b) What do you do when you play?

c) How does gameplay generally, or playing GTA V specifically, fit into your everyday life?

d) How do your family/friends feel about you play, do you play only when you are in a certain mood, do you talk to your friends about your gameplay?

2. How do you feel when you play? (prompt: physically, emotionally, mentally)

3. If you had to describe what this videogame means to you, what would you say? (prompt: What words come to mind, what images? How do you talk about its importance to others?)

\section{Identity}

4. How would you describe yourself as a person? (Prompt: What sort of person are you? Most important characteristics)

5. Does playing games make a difference in how you see yourself? (prompt: If so, how do you see yourself now as different from non-gamers?)

a) Would you describe yourself as a Gamer? What does or does not make you a gamer? If not, why don't you identify this way?

6. Did you feel that you could identify with the characters in the game? Can you explain?

a) there other characters you identify with strongly, what about them helps make that connection?).

\section{Violence and Intensity}

7. Can you tell me about a moment, feelings or scene that was particularly memorable to you or that stood out while you were playing?

a) How did these moments make you feel?

b) Have you thought about these moments since playing the game? In what context, or why? 
8. What do you think about the role of violence in GTA V?

a. Do you talk to others (family, friends, online forums) about these moments?

9. What part of the game was most pleasurable?

\section{Position in Feminist Discourse}

10. Does your experience correlate with the way GTA V is talked about? Why or why not? (prompt: how does this make you feel?)

a. How would you like the media to talk about the game? 